\title{
The linear fractional model on the ball
}

Frédéric Bayart

\begin{abstract}
Given a holomorphic self-map $\varphi$ of the ball of $\mathbb{C}^{N}$, we study whether there exists a map $\sigma$ and a linear fractional transformation $A$ such that $\sigma \circ \varphi=A \circ \sigma$. This is an important result when $N=1$ with a great number of applications. We extend this result to the multi-dimensional setting for a large class of maps. Applications to commuting holomorphic self-maps are given.
\end{abstract}

\section{Introduction}

The story of this paper goes back to Königs [17] in 1884: given a self-map $\varphi$ of the unit disk $\mathbb{D}$, fixing 0 and satisfying $\lambda=\varphi^{\prime}(0) \neq 0$, he proved that there exists a (non-zero) holomorphic function $\sigma: \mathbb{D} \rightarrow \mathbb{C}$ satisfying

$$
\sigma \circ \varphi=\lambda \sigma
$$

When the map $\varphi$ does not fix any point in $\mathbb{D}$, the situation becomes more involved. It is well known that in this case, there exists an unique $\tau$ on the circle $\mathbb{T}$ such that $\varphi$ has nontangential limit $\tau$ at $\tau$ and $\varphi^{\prime}$ has nontangential limit $\varphi^{\prime}(\tau)=\lambda_{\tau} \in(0,1]$ at $\tau: \tau$ is the Denjoy-Wolff point of $\varphi$ and $\lambda_{\tau}$ is called the boundary dilation coefficient of $\varphi$ at $\tau$. If $\lambda_{\tau}<1$, then the function is called hyperbolic, otherwise, it is called parabolic. Conjugating by an automorphism if necessary, we may always assume that $\tau=1$.

The problem is more clear on the right half-plane $\mathbb{H}=\{z \in \mathbb{C}: \Re(z)>0\}$. Setting $U(z)=\frac{1+z}{1-z}$ the Cayley transform which maps conformally $\mathbb{D}$ onto $\mathbb{H}$ and $\phi=U \circ \varphi \circ U^{-1}, \phi$ is a self-map of $\mathbb{H}$ fixing $\infty$. For the hyperbolic case, Valiron [23] proved in 1931 that there remains a solution to (1.1). However, there is no solution in the parabolic case, and we are led to solve the Abel's

2000 Mathematics Subject Classification: 32H50, 32A10.

Keywords: Linear fractional maps, iteration. 
equation: to find a holomorphic function $\sigma: \mathbb{H} \rightarrow \mathbb{C}$ and a complex number $a$ such that

$$
\sigma \circ \phi=T_{a} \circ \sigma
$$

where $T_{a}$ is the translation $T_{a}(z)=z+a$. This was done by several authors at the end of the 1970's (see [19], [3], [12]). We say that $(\sigma, \lambda z)$ or $\left(\sigma, T_{a}\right)$ is a linear fractional model for $\phi$ since dilations and translations are the single linear fractional maps of $\mathbb{C}$ fixing $\infty$.

The existence of a linear fractional model for self-maps of $\mathbb{D}$ has many applications, concerning for instance the dynamics of the map itself, the study of families of commuting mappings or questions related to composition operators associated to a given map. Moreover, it turns out that the more information one has on $\sigma$, the more useful the model is. In this vein, we have to speak about the work of Bourdon and Shapiro [6], where under some regularity assumption of $\varphi$ at the Denjoy-Wolff point, more accurate information on the behavior of $\sigma$ near infinity is given.

Recently Cowen and MacCluer have introduced in [13] a class of selfmaps of the unit ball $\mathbb{B}_{N}$ of $\mathbb{C}^{N}$, which seems to be a good generalization of the linear fractional maps of $\mathbb{D}$. They called them the linear fractional maps of the ball. The hope of these authors was that these maps would play a similar role in several variables. In particular, the existence of a linear fractional model for self-maps of the ball seems to be a central problem in this circle of ideas.

In this work, we are concerned with the case $N=2$. We try to extend Bourdon-Shapiro's work for self-maps of the ball $\mathbb{B}_{2}$. Namely, we would like to obtain a linear fractional model for self-maps $\varphi$ of $\mathbb{B}_{2}$, with no inner fixed points and some regularity conditions at the Denjoy-Wolff point $\tau$. In the following, we will always assume that $\tau$ is equal to $e_{1}=(1,0)$. Our first problem in Section 2 is to give an appropriate notion of regularity at $e_{1}$. We will explain why the classical notion of $\mathcal{C}^{m}$-regularity is not the best choice in this context. Instead of it, we will provide a (related) notion of $\mathcal{D}^{m_{-}}$ regularity. Next, we will carefully study how this notion of regularity at $e_{1}$ transfers into a notion of regularity at $\infty$, when $\mathbb{B}_{2}$ is mapped on the Siegel half-plane. Indeed, as for the one-dimensional case, it is more convenient to work on the Siegel half-plane where the geometry of linear fractional maps is easier to describe.

Suppose now that $\varphi$ is differentiable at the Denjoy-Wolff point $e_{1}$. Since the problem of the existence of a linear fractional model is invariant under conjugation by an automorphism, we may reduce $d \varphi_{e_{1}}$. Basically, there are three cases to consider: 
1. the hyperbolic case, when after reduction

$$
d \varphi_{e_{1}}=\left(\begin{array}{cc}
\lambda_{1} & 0 \\
\alpha_{1} & \mu_{1}
\end{array}\right) \quad \text { with } \lambda_{1} \in(0,1)
$$

2. the parabolic diagonalizable case, when after reduction $d \varphi_{e_{1}}=\left(\begin{array}{cc}1 & 0 \\ 0 & \mu_{1}\end{array}\right)$.

3. the parabolic non-diagonalizable case, when after reduction

$$
d \varphi_{e_{1}}=\left(\begin{array}{cc}
1 & 0 \\
\alpha_{1} & 1
\end{array}\right), \quad \text { with } \alpha_{1} \neq 0 .
$$

At a first sight, one might think that the parabolic non-diagonalizable case should be the most difficult one. This is not the case, and in Section 3 we will prove that a linear fractional model does exist under weak assumptions on the regularity of $\varphi$. The model map here is a generalized Heisenberg translation (see [4]) and we will be able to give some informations on the regularity of the intertwining map.

The hyperbolic case was already considered by Bracci and Gentili in [9] under a (much more) restrictive condition on the regularity of $\varphi$ and when $\left|\mu_{1}\right|>\lambda_{1}^{3 / 4}$. In Section 4, we will prove that a linear fractional model exists for such maps in almost every case. However, the model map will be more and more difficult to exhibit when $\left|\mu_{1}\right|$ decreases. In particular, the regularity needed on $\varphi$ for the existence of a linear fractional model will depend on the value of $\left|\mu_{1}\right|$. Moreover we will show that when certain algebraic relations appear between $\mu_{1}$ and $\lambda_{1}$, there is no hope to obtain a linear fractional model under reasonable conditions. Instead of it, we will provide another model map which is not a linear fractional transformation. This problem of the existence of algebraic relations between eigenvalues (called resonances) already appears in the context of differential equations in the Poincaré-Dulac Theorem (see for instance [21]).

The parabolic diagonalizable case seems to be the most difficult one. We will give some indications for it in Section 5, like the linearization of the first coordinate when $\left|\mu_{1}\right|<1$ and a complete linear fractional model for several kinds of maps. Here our techniques involve the use of special functions, especially the Lerch transcendent function. Section 6 will be devoted to applications, in particular to the study of commuting self-maps of the ball. Throughout this paper, several open questions will be asked. We end up this introduction by noting that the study of (1.1) in the case of an inner fixed point was done in [14].

Acknowledgement. We are grateful to the referee for his/her careful reading and many valuable suggestions which improved the presentation of this paper. 


\section{Preliminary}

\subsection{Denjoy-Wolff and Julia-Caratheodory}

Let $\mathbb{B}_{2}=\left\{Z=(w, z) \in \mathbb{C}^{2}:\|Z\|^{2}=|w|^{2}+|z|^{2}<1\right\}$ be the unit ball of $\mathbb{C}^{2}$ and let $\varphi: \mathbb{B}_{2} \rightarrow \mathbb{B}_{2}$ be holomorphic. The iterates of $\varphi$ are denoted by $\varphi^{[n]}=\varphi \circ \cdots \circ \varphi$. MacCluer has extended in [18] the Denjoy-Wolff theorem to self-maps of $\mathbb{B}_{2}$ : suppose that $\varphi$ has no fixed points in $\mathbb{B}_{2}$. Then there exists an unique $\xi \in \partial \mathbb{B}_{2}$ such that $\varphi^{[n]}$ converges uniformly on compact subsets of $\mathbb{B}_{2}$ to $\xi$. Conjugating by an automorphism if necessary, we will always assume that $\xi=e_{1}=(1,0)$. At the Denjoy-Wolff point $e_{1}$, the boundary dilation coefficient of $\varphi$, defined by $\liminf _{Z \rightarrow e_{1}}(1-\|\varphi(Z)\|) /(1-\|Z\|)$ belongs to $(0,1]$. Hence, it is possible to apply the version of the Julia-Caratheodory theorem for the ball, due to Rudin, which is recalled here in the form which is useful for us.

Theorem 2.1 ([20], [1]). Let $\varphi$ be a (holomorphic) self-map of $\mathbb{B}_{2}$ with no inner fixed point and suppose that $e_{1}$ is its Denjoy-Wolff point. Let $\lambda(\varphi)$ be the boundary dilation coefficient of $\varphi$ at $e_{1}$. Then $\frac{1-\varphi_{1}(w, z)}{1-w}$ is bounded in every Korányi region. Moreover, it has restricted $K$-limit $\lambda(\varphi)$ at $e_{1}$.

Suppose now that $\varphi$ has an expansion at its Denjoy-Wolff point up to the second order,

$$
\begin{gathered}
\varphi(w, z)=\left(1+\lambda_{1}(w-1)+\beta_{1} z+\gamma_{1} z^{2}+a z(w-1)+b(w-1)^{2}+\varepsilon_{1}(w, z),\right. \\
\left.\alpha_{1}(w-1)+\mu_{1} z+a^{\prime} z^{2}+b^{\prime} z(w-1)+c^{\prime}(w-1)^{2}+\varepsilon_{2}(w, z)\right)
\end{gathered}
$$

where $\varepsilon_{i}(w, z)=o\left(\|(w-1, z)\|^{2}\right)$. Observe that we have used greek letters and roman letters. The notation for greek letters will be fixed during all this paper, whereas the notation for roman letters will change. If the context is not clear, then we will write $\lambda_{1}^{\varphi}, \mu_{1}^{\varphi}, \ldots$ instead of $\lambda_{1}, \mu_{1} \ldots$

Taking $w_{n}=1-1 / n$ and $z_{n}=0$, the Julia-Caratheodory theorem ensures that

$$
\lambda_{1}=\lim _{n \rightarrow+\infty} \frac{1-\varphi_{1}\left(w_{n}, z_{n}\right)}{1-w_{n}}=\lambda(\varphi) \in(0,1] .
$$

Moreover, if $\beta_{1} \neq 0$, if we take $w_{n}=1-1 / n$ and $z_{n}=e^{i n \theta} / \sqrt{n}$ where $e^{i \theta} \beta_{1}=\left|\beta_{1}\right|$, it is easy to check that $\left|\varphi_{1}\left(w_{n}, z_{n}\right)\right|>1$ for $n$ large enough, which is impossible. Finally, for this choice of $\left(w_{n}, z_{n}\right)$, with now $\gamma_{1} e^{i 2 \theta}=\left|\gamma_{1}\right|$, one has

$$
\begin{aligned}
\left\|\left(w_{n}, z_{n}\right)\right\|^{2} & =1-1 / n+o(1 / n) \\
\left\|\varphi\left(w_{n}, z_{n}\right)\right\|^{2} & =1+\left(\left|\mu_{1}\right|^{2}-2 \lambda_{1}+2\left|\gamma_{1}\right|\right) / n+o(1 / n) .
\end{aligned}
$$


Since $\lambda_{1}=\lambda(f)$, one has $2 \lambda_{1}-2\left|\gamma_{1}\right|-\left|\mu_{1}\right|^{2} \geq \lambda_{1}$, namely

$$
\lambda_{1} \geq\left|\mu_{1}\right|^{2}+2\left|\gamma_{1}\right| \text {. }
$$

Remark 2.2. In general we do not have $\gamma_{1}=0$. For instance, the map $\varphi(w, z)=\left(1+(w-1) / 2+z^{2} / 16, z / 2\right)$ maps $\mathbb{B}_{2}$ into $\mathbb{B}_{2}$, has $e_{1}$ as DenjoyWolff point and $\gamma_{1}=1 / 16 \neq 0$. In [9], the work on the hyperbolic case was done assuming $\gamma_{1}=0$ (see [9, Remark 1.6]). This explains in part why our paper works in a more general context.

Another useful result is Wolff's lemma for the ball.

Theorem 2.3. [20, Theorem 8.5.3] Let $\varphi$ be a (holomorphic) self-map of $\mathbb{B}_{2}$ with no inner fixed point and suppose that $e_{1}$ is its Denjoy-Wolff point. Let $\lambda_{1}$ be the boundary dilation coefficient of $\varphi$ at $e_{1}$. Then $\varphi$ maps a horosphere

$$
E(\alpha)=\left\{Z=(w, z) \in \mathbb{B}_{2}: \frac{|1-w|^{2}}{1-\|Z\|^{2}}<\alpha\right\}, 0<\alpha<1,
$$

into the horosphere $E\left(\lambda_{1} \alpha\right)$.

\subsection{Regularity at $e_{1}$ and at infinity}

Let $\varphi$ be a self-map of $\mathbb{B}_{2}$ whose Denjoy-Wolff point is $e_{1}$. The most natural notion of regularity at $e_{1}$ is probably the following.

Definition 2.4. We say that $\varphi$ belongs to $\mathcal{C}^{m}\left(e_{1}\right), m \geq 1$, provided it can be written

$$
\begin{gathered}
\varphi(w, z)=\left(1+\lambda_{1}(w-1)+\sum_{\substack{j+k \geq 2 \\
j+k \leq m}} a_{j, k} z^{j}(w-1)^{k}+\varepsilon_{1}(w, z),\right. \\
\left.\alpha_{1}(w-1)+\mu_{1} z+\sum_{\substack{j+k \geq 2 \\
j+k \leq m}} b_{j, k} z^{j}(w-1)^{k}+\varepsilon_{2}(w, z)\right)
\end{gathered}
$$

with $\varepsilon_{i}(w, z)=o\left(\|(w-1, z)\|^{m}\right)$. We define in the same way the class $\mathcal{C}^{m+\varepsilon}\left(e_{1}\right), m \geq 1,0<\varepsilon<1$, by assuming $\varepsilon_{i}(w, z)=O\left(\|(w-1, z)\|^{m+\varepsilon}\right)$.

However, in the ball near $e_{1}$, the quantities $|z|$ and $|w-1|$ do not play the same role (this was already used in the previous subsection). Indeed, one has $|z|^{2} \leq 1-|w|^{2} \leq 2|1-w|$. This leads us to the following definition: 
Definition 2.5. We say that $\varphi$ belongs to $\mathcal{D}^{m}\left(e_{1}\right), m \geq 2$, provided it can be written

$$
\begin{gathered}
\varphi(w, z)=\left(1+\lambda_{1}(w-1)+\sum_{\substack{j+k \geq 2 \\
j / 2+k \leq m / 2}} a_{j, k} z^{j}(w-1)^{k}+\varepsilon_{1}(w, z),\right. \\
\left.\alpha_{1}(w-1)+\mu_{1} z+\sum_{\substack{j+k \geq 2 \\
j / 2+k \leq m / 2}} b_{j, k} z^{j}(w-1)^{k}+\varepsilon_{2}(w, z)\right)
\end{gathered}
$$

with $\varepsilon_{i}(w, z)=o\left(|w-1|^{m / 2}\right)$. We define in the same way the class $\mathcal{D}^{m+\varepsilon}\left(e_{1}\right)$, $m \geq 1,0<\varepsilon<1$, by assuming $\varepsilon_{i}(w, z)=O\left(|w-1|^{(m+\varepsilon) / 2}\right)$.

Clearly, the following inclusions hold true: $\mathcal{C}^{m}\left(e_{1}\right) \subset \mathcal{D}^{m}\left(e_{1}\right)$ and $\mathcal{D}^{2 m}\left(e_{1}\right)$ $\subset \mathcal{C}^{m}\left(e_{1}\right)$.

As for the one-dimensional case, the existence of a linear fractional model will be more clear if we transfer the problem on a half-plane. Let $\mathbb{H}_{2}=$ $\left\{(w, z) \in \mathbb{C}^{2}: \Re(w)>|z|^{2}\right\}$ be the Siegel half-plane, and let $U(w, z)=$ $\left(\frac{w+1}{1-w}, \frac{z}{1-w}\right)$ be the Cayley transform. $U$ is a biholomorphic map from $\mathbb{B}_{2}$ onto $\mathbb{H}_{2}$, which extends to a homeomorphism from $\overline{\mathbb{B}_{2}}$ onto $\overline{\mathbb{H}_{2}} \cup\{\infty\}$. Its reciprocal is given by $U^{-1}(w, z)=\left(\frac{w-1}{w+1}, \frac{2 z}{w+1}\right)$. Let $\phi=U \circ \varphi \circ U^{-1}$, which is a self-map of $\mathbb{H}_{2}$ whose Denjoy-Wolff point is $\infty$. The boundary dilation coefficient of $\phi$ at $\infty$ is $\lambda_{\infty}=1 / \lambda_{1}$ and Wolff's lemma becomes: every horosphere $\mathbb{H}_{2, R}=\left\{(w, z) \in \mathbb{H}_{2}: \Re(w)>|z|^{2}+R\right\}$ is mapped under $\phi$ into the horosphere $\mathbb{H}_{2, \lambda_{\infty} R}$.

Suppose now that $\varphi$ belongs to $\mathcal{D}^{m+\varepsilon}\left(e_{1}\right)$. One has to study how this can be translated on the expansion of $\phi$ near $\infty$. The situation is more involved than that on the disk; in particular, one of the main problems comes from the inequality $\gamma_{1} \neq 0$. For the first coordinate, one has:

$$
\begin{aligned}
\phi_{1}(w, z) & =-1+\frac{2}{1-\left(\varphi \circ U^{-1}(w, z)\right)_{1}} \\
& =-1+\frac{2}{\frac{2 \lambda_{1}}{w+1}-\sum_{\substack{j+k \geq 2 \\
j / 2+k \leq m / 2}} a_{j, k} \frac{2^{j+k}(-1)^{k} z^{j}}{(w+1)^{j+k}}+\varepsilon_{1}^{\prime}(w, z)}
\end{aligned}
$$

where

$$
\varepsilon_{1}^{\prime}(w, z)=\varepsilon_{1}\left(\frac{w-1}{w+1}, \frac{2 z}{w+1}\right)
$$

satisfies $\varepsilon_{i}^{\prime}(w, z)=O\left(|w+1|^{-(m+\varepsilon) / 2}\right)$. Setting $a_{j, k}^{\prime}=\frac{2^{j+k-1}(-1)^{k}}{\lambda_{1}} a_{j, k}$, we get $\phi_{1}(w, z)=-1+\left(\frac{w+1}{\lambda_{1}}\right)\left(1+\sum_{l \geq 1}\left(\sum_{\substack{j+k \geq 2 \\ j / 2+k \leq m / 2}} a_{j, k}^{\prime} \frac{z^{j}}{(w+1)^{j+k-1}}\right)^{l}+\varepsilon_{1}^{\prime \prime}(w, z)\right)$ with $\varepsilon_{1}^{\prime \prime}(w, z)=O\left(\frac{1}{|w+1|^{(m+\varepsilon) / 2-1}}\right)$. 
Let us set $\gamma_{\infty}=a_{2,0}^{\prime}=\frac{2 a_{2,0}}{\lambda_{1}}=\frac{2 \gamma_{1}}{\lambda_{1}}$ and $\lambda_{\infty}=\frac{1}{\lambda_{1}}$. In $\left(\sum \ldots\right)^{l}$, we only keep the terms which are not smaller than $|w+1|^{1-(m+\varepsilon) / 2}$. For this, we write

$$
\sum_{\substack{j+k \geq 2 \\ j / 2+k \leq m / 2}} a_{j, k}^{\prime} \frac{z^{j}}{(w+1)^{j+k-1}}=\gamma_{\infty} \frac{z^{2}}{w+1}+A(w, z),
$$

where $A(w, z)=O\left(|w+1|^{-1 / 2}\right)$. Hence we obtain

$$
\sum_{l \geq 1}\left(\sum \ldots\right)^{l}=\sum_{l \geq 1}^{\max (m-2, l)} \sum_{r=0}^{r} C_{l}^{r}\left(\frac{\gamma_{\infty} z^{2}}{w+1}\right)^{l-r} A(w, z)^{r}+O\left(|w+1|^{1-(m+\varepsilon) / 2}\right) .
$$

$A(w, z)^{r}$ is a sum of terms like $\frac{z^{p}}{(w+1)^{q}}$. We just keep the terms with $q-p / 2 \leq$ $m / 2-1$ (the remaining terms are $O\left(|w+1|^{1-(m+\varepsilon) / 2}\right)$ ). Moreover, such a couple $(p, q)$ comes from a product of $r$ terms, and one has $p=j_{1}+\cdots+j_{r}$, $q=j_{1}+\cdots+j_{r}+k_{1}+\cdots+k_{r}-r$ with $j_{i}+k_{i} \geq 2$ and $k_{i}=0 \Longrightarrow j_{i} \geq 3$. Let $u=\operatorname{card}\left\{i: k_{i}=0\right\}$. One has $q-p=k_{1}+\cdots+k_{r}-r \geq-u$. On the other hand, the inequality $j_{i} \geq 3$ provided $k_{i}=0$ gives $p \geq 3 u$. We finally obtain $p \leq \frac{3 q}{2}$. Let us set

$$
\mathcal{E}_{m}=\left\{(p, q) \in \mathbb{N}^{2}:(p, q) \neq(0,0), q \leq p / 2+m / 2-1 \text { and } p \leq \frac{3 q}{2}\right\} .
$$

$\mathcal{E}_{m}$ is a finite subset of $\mathbb{N}^{2}$. If we arrange the terms of (2.2) in a suitable way (in particular we change the orders of summation), then we find

$$
\begin{aligned}
1+\sum_{l \geq 1} & \left(\sum \ldots\right)^{l}= \\
= & \frac{1}{1-\gamma_{\infty} \frac{z^{2}}{w+1}}+\sum_{(p, q) \in \mathcal{E}_{m}} \frac{z^{p}}{(w+1)^{q}} \sum_{r=1}^{m-2} d_{p, q, r} \sum_{l \geq r} C_{l}^{r}\left(\gamma_{\infty} \frac{z^{2}}{w+1}\right)^{l-r} \\
& +O\left(|w+1|^{1-(m+\varepsilon) / 2}\right)
\end{aligned}
$$

where $d_{p, q, r}$ belongs to $\mathbb{C}$. Observe that for each $p, q$, the function

$$
u \mapsto \sum_{r=1}^{m-2} d_{p, q, r} \sum_{l \geq r} C_{l}^{r}\left(\gamma_{\infty} u\right)^{l-r}
$$

is a continuous function on $\mathbb{D}$ since $\left|\gamma_{\infty}\right| \leq 1$. It reduces to a constant as soon as $\gamma_{\infty}=0$. Finally, if we come back to $\phi_{1}$, we get

$\phi_{1}(w, z)=\frac{\lambda_{\infty} w}{1-\gamma_{\infty} \frac{z^{2}}{w+1}}+\sum_{(p, q) \in \mathcal{E}_{m}} E_{p, q}\left(\frac{z^{2}}{w+1}\right) \frac{z^{p}}{(w+1)^{q-1}}+O\left(\frac{1}{|w+1|^{(m+\varepsilon) / 2-2}}\right)$.

$E_{p, q}$ is analytic on $\mathbb{D}$ and is constant if $\gamma_{\infty}=0$. Observe that if we assume $\mu_{1} \neq 0$, then one has $\left|\gamma_{\infty}\right|<1$ and the functions $E_{p, q}$ are continuous on $\overline{\mathbb{D}}$. 
The statement for the second coordinate follows the same lines. Indeed one has

$$
\phi_{2}(w, z)=\frac{\mu_{1} z-\alpha_{1}+\sum_{\substack{j+k \geq 2 \\ j / 2+k \leq m / 2}} b_{j, k} \frac{2^{j+k-1}(-1)^{k}}{(w+1)^{j+k-1}}+O\left(|w+1|^{1-(m+\varepsilon) / 2}\right)}{\lambda_{1}\left(1+\sum_{\substack{j+k \geq 2 \\ j / 2+k \leq m / 2}}^{\prime} a_{j, k}^{\prime} \frac{z^{j}}{(w+1)^{j+k-1}}+O\left(|w+1|^{(1-(m+\varepsilon) / 2)}\right)\right)} .
$$

We set $\mu_{\infty}=\mu_{1} / \lambda_{1}, \alpha_{\infty}=\alpha_{1} / \lambda_{1}$ and $b_{j, k}^{\prime}=\frac{2^{j+k-1}(-1)^{k}}{\lambda_{1}} b_{j, k}$. This gives:

$$
\begin{aligned}
& \phi_{2}(w, z)=\left(\mu_{\infty} z-\alpha_{\infty}+\sum_{\substack{j+k \geq 2 \\
j / 2+k \leq m / 2}} b_{j, k}^{\prime} \frac{z^{j}}{(w+1)^{j+k}}\right) \times \\
& \left(\frac{1}{1-\gamma_{\infty} \frac{z^{2}}{w+1}}+\sum_{(p, q) \in \mathcal{E}_{m}} E_{p, q}^{\prime}\left(\frac{z^{2}}{w+1}\right) \frac{z^{p}}{(w+1)^{q}}\right)+O\left(|w+1|^{-1+(m+\varepsilon) / 2}\right) \\
& =\frac{\mu_{\infty} z}{1-\gamma_{\infty} \frac{z^{2}}{w+1}}-\frac{\alpha_{\infty}}{1-\gamma_{\infty} \frac{z^{2}}{w+1}}+\sum_{(u, v) \in \mathcal{F}_{m}} F_{u, v}\left(\frac{z^{2}}{w+1}\right) \frac{z^{u}}{(w+1)^{v}}+ \\
& \quad+O\left(|w+1|^{-1+(m+\varepsilon) / 2}\right)
\end{aligned}
$$

where each $F_{u, v}$ is continuous on $\mathbb{D}$ and constant if $\gamma_{\infty}=0 . u$ and $v$ are obtained by $u=j+p, v=j+k-1+q$. If we observe that

$$
\frac{3 v}{2}-u \geq \frac{3 j+3 k-3}{2}-j \geq-1
$$

we may take for $\mathcal{F}_{m}$ the set

$$
\mathcal{F}_{m}=\left\{(u, v) \in \mathbb{N}^{2}: v \neq 0, u \leq \frac{3 v}{2}+1 \text { and } v \leq u / 2+m / 2-1\right\} .
$$

The following proposition summarizes our work:

Proposition 2.6. Let $\varphi$ be in $\mathcal{D}^{m}\left(e_{1}\right)$ (resp. in $\mathcal{D}^{m+\varepsilon}\left(e_{1}\right)$ ) and $\phi=U \circ \varphi \circ U^{-1}$. Then $\phi$ may be written:

$$
\begin{aligned}
& \phi_{1}(w, z)=\frac{\lambda_{\infty} w}{1-\gamma_{\infty} \frac{z^{2}}{w+1}}+\sum_{(p, q) \in \mathcal{E}_{m}} E_{p, q}\left(\frac{z^{2}}{w+1}\right) \frac{z^{p}}{(w+1)^{q-1}}+\varepsilon_{1}(w, z), \\
& \phi_{2}(w, z)=\frac{\mu_{\infty} z}{1-\gamma_{\infty} \frac{z^{2}}{w+1}}-\frac{\alpha_{\infty}}{1-\gamma_{\infty} \frac{z^{2}}{w+1}}+\sum_{(u, v) \in \mathcal{F}_{m}} F_{u, v}\left(\frac{z^{2}}{w+1}\right) \frac{z^{u}}{(w+1)^{v}}+\varepsilon_{2}(w, z),
\end{aligned}
$$

where $\lambda_{\infty}=1 / \lambda_{1}, \mu_{\infty}=\mu_{1} / \lambda_{1}, \gamma_{\infty}=2 \gamma_{1} / \lambda_{1}, \alpha_{\infty}=\alpha_{1} / \lambda_{1}$. The functions $E_{p, q}$ and $F_{u, v}$ are analytic on $\mathbb{D}$ and even continuous on $\overline{\mathbb{D}}$ provided $\mu_{1} \neq 0$. They are constant if $\gamma_{1}=0$. Moreover, one has $\varepsilon_{1}(w, z)=o\left(|w+1|^{2-m / 2}\right)$ $\left(\right.$ resp. $\varepsilon_{1}(w, z)=O\left(|w+1|^{2-(m+\varepsilon) / 2}\right)$ and $\varepsilon_{2}(w, z)=o\left(|w+1|^{1-m / 2}\right)$ (resp. $\left.\varepsilon_{2}(w, z)=O\left(|w+1|^{1-(m+\varepsilon) / 2}\right)\right)$. 
It is worth noting that (2.1) translates with these new notations into

$$
\lambda_{\infty} \geq\left|\mu_{\infty}\right|^{2}+\left|\gamma_{\infty}\right| \lambda_{\infty}
$$

Sometimes the previous way for writing $\phi_{1}$ and $\phi_{2}$ will not be convenient. In particular, the iteration will be easier to compute without the factor $\frac{1}{1-\gamma_{\infty} \frac{z^{2}}{w+1}}$ and the estimations easier to obtain with $w$ instead of $w+1$. Now, it is almost trivial to observe that for $|w|>1$,

$$
E_{p, q}\left(\frac{z^{2}}{w+1}\right) \frac{z^{p}}{(w+1)^{q-1}}=E_{p, q}\left(\frac{z^{2}}{w}\right) \frac{z^{p}}{w^{q-1}}+\sum_{\substack{(u, v) \in \mathcal{E}_{m} ; \\ u-2 v<p-2 q}} E_{u, v}^{\prime}\left(\frac{z^{2}}{w}\right) \frac{z^{u}}{w^{v}}+\varepsilon(w, z)
$$

where $E_{u, v}^{\prime}$ is analytic in $\mathbb{D}$ and continuous on $\overline{\mathbb{D}}$ and $|\varepsilon(w, z)|=O\left(|w|^{2-(m+1) / 2}\right)$. Indeed, we expand $E_{p, q}(u)=\sum_{l} a_{l} u^{l}$ and we write

$$
\begin{aligned}
E_{p, q}\left(\frac{z^{2}}{w+1}\right) & \frac{z^{p}}{(w+1)^{q-1}}= \\
& =\sum_{l} a_{l}\left(\frac{z^{2}}{w}\right)^{l}\left(1+\sum_{r \geq 1} \frac{(-1)^{r}}{w^{r}}\right)^{l} \frac{z^{p}}{w^{q-1}}\left(1+\sum_{r \geq 1} \frac{(-1)^{r}}{w^{r}}\right)^{q-1} .
\end{aligned}
$$

A suitable reorganization of the terms shows the claim. We shall do the same for $\frac{\lambda_{\infty} w}{1-\gamma_{\infty} \frac{z^{2}}{w+1}}$ which becomes

$$
\lambda_{\infty} w+z^{2} E_{2,1}\left(\frac{z^{2}}{w}\right)+\sum_{(p, q) \in \mathcal{E}_{m}} E_{p, q}^{\prime \prime}\left(\frac{z^{2}}{w}\right)+\varepsilon(w, z) .
$$

Finally a similar action on $\phi_{2}$ gives rise to the following corollary.

Corollary 2.7. Let $\varphi$ be in $\mathcal{D}^{m}\left(e_{1}\right)$ (resp. in $\mathcal{D}^{m+\varepsilon}\left(e_{1}\right)$ ) and $\phi=U \circ \varphi \circ U^{-1}$. Then $\phi$ may be written:

$$
\begin{aligned}
& \phi_{1}(w, z)=\lambda_{\infty} w+z^{2} E_{2,1}\left(\frac{z^{2}}{w}\right)+\sum_{(p, q) \in \mathcal{E}_{m}} E_{p, q}\left(\frac{z^{2}}{w}\right) \frac{z^{p}}{w^{q-1}}+\varepsilon_{1}(w, z), \\
& \phi_{2}(w, z)=\mu_{\infty} z-\alpha_{\infty}+\frac{z^{3}}{w} F_{3,1}\left(\frac{z^{2}}{w}\right)+\sum_{(u, v) \in \mathcal{F}_{m}} F_{u, v}\left(\frac{z^{2}}{w}\right) \frac{z^{u}}{w^{v}}+\varepsilon_{2}(w, z) .
\end{aligned}
$$

The functions $E_{p, q}$ and $F_{u, v}$ are analytic on $\mathbb{D}$ and even continuous on $\overline{\mathbb{D}}$ provided $\mu_{1} \neq 0$. They are constant if $\gamma_{1}=0$ with $F_{3,1}=E_{2,1}=0$. Moreover, one has $\varepsilon_{1}(w, z)=o\left(|w|^{2-m / 2}\right)$ (resp. $\varepsilon_{1}(w, z)=O\left(|w|^{2-(m+\varepsilon) / 2}\right)$ and $\varepsilon_{2}(w, z)=o\left(|w|^{1-m / 2}\right)\left(\right.$ resp. $\left.\varepsilon_{2}(w, z)=O\left(|w|^{1-(m+\varepsilon) / 2}\right)\right)$. 


\subsection{The three cases}

Suppose that $\varphi \in \mathcal{D}^{2}\left(e_{1}\right)$ and set

$$
d \varphi_{e_{1}}=\left(\begin{array}{cc}
\lambda_{1} & 0 \\
\alpha_{1} & \mu_{1}
\end{array}\right)
$$

It is obvious that if $\psi$ is an automorphism of $\mathbb{B}_{2}$ fixing $e_{1}$, then one has $d\left(\psi^{-1} \circ \varphi \circ \psi\right)_{e_{1}}=d \psi_{e_{1}}^{-1} \circ d \varphi_{e_{1}} \circ d \psi_{e_{1}}$. Now, there are automorphisms of $\mathbb{B}_{2}$ fixing $e_{1}$ (the Heisenberg translations) whose differential at $e_{1}$ is equal to $\left(\begin{array}{ll}1 & 0 \\ a & 1\end{array}\right)=M_{a}$. Moreover, provided $\lambda_{1} \neq \mu_{1}$, it is possible to choose $a=$ $\alpha_{1} /\left(\lambda_{1}-\mu_{1}\right)$ such that

$$
M_{a}^{-1} d \varphi_{e_{1}} M_{a}=\left(\begin{array}{cc}
\lambda_{1} & 0 \\
0 & \mu_{1}
\end{array}\right)
$$

Thus, we distinguish three cases:

1. the hyperbolic case, $\lambda_{1}<1$. If $\mu_{1} \neq \lambda_{1}$ (or equivalently if $\mu_{\infty} \neq 1$ ), it will be possible to assume $\alpha_{1}=0$.

2. the parabolic diagonalizable case, $\lambda_{1}=1$ and by conjugation if necessary,

$$
d \varphi_{e_{1}}=\left(\begin{array}{cc}
1 & 0 \\
0 & \mu_{1}
\end{array}\right)
$$

This happens in particular if $\mu_{1} \neq 1$.

3. the parabolic nondiagonalizable case, $\lambda_{1}=\mu_{1}=1$ and $\alpha_{1} \neq 0$.

\subsection{Linear fractional maps of $\mathbb{H}_{2}$}

Following [13], a linear fractional map of $\mathbb{C}^{2}$ is defined as a map of $\mathbb{C}^{2}$ which may be written $\psi(Z)=\frac{A Z+B}{\langle Z, C\rangle+d}$ where $A \in M_{2}(\mathbb{C}), B, C \in \mathbb{C}^{2}$ and $d \in \mathbb{C}$. If $\psi$ is a self-map of the domain $U$, we will write $\psi \in \operatorname{LFM}(U)$. The linear fractional maps of the Siegel half-plane with $\infty$ as attractive fixed point have been determined in [8]. They can be written

$$
\psi(w, z)=(\lambda w+a z+b, \mu z+c)
$$

with $\lambda \geq 1,|\mu|^{2} \leq \lambda$ and some other conditions on $a, b, c$. In particular, if $|\mu|^{2}=\lambda$, these conditions become

$$
\Re(b) \geq|c|^{2}, a=2 \mu \bar{c} .
$$


When $\mu \neq 1$, we can conjugate $\psi$ by a Heisenberg translation (i.e. an automorphism of $\mathbb{H}_{2}$ sending $(w, z)$ on $(w+2 \bar{\alpha} z+\beta, z+\alpha)$ with $\left.\Re(\beta)=|\alpha|^{2}\right)$ to ensure that $c=0$. Hence, we can classify the linear fractional map following their derivative at the Denjoy-Wolff point. This is done in the forthcoming table. Since our approach is based on a careful study of the orbits of $(w, z) \in \mathbb{H}_{2}$ under $\phi$, we also include in this table a supplementary information: for $(w, z) \in \mathbb{H}_{2}$, let $(w(n), z(n))=\psi^{[n]}(w, z)$. The behavior of $(w(n), z(n))$ is a key tool for our work.

\begin{tabular}{c|c|c} 
Derivative & Model map & Behavior of orbits \\
\hline$\left(\begin{array}{ll}\lambda & 0 \\
0 & \mu\end{array}\right), \lambda>1, \mu \neq 1$ & $\psi(w, z)=(\lambda w, \mu z)$ & $\begin{array}{c}w(n) \sim \lambda^{n} w \\
z(n) \sim \mu^{n} z .\end{array}$ \\
\hline$\left(\begin{array}{ll}\lambda & 0 \\
0 & 1\end{array}\right), \lambda>1$ & $\psi(w, z)=(\lambda w, z+a)$ & $\begin{array}{c}w(n) \sim \lambda^{n} w \\
z(n) \sim n a .\end{array}$ \\
\hline$\left(\begin{array}{ll}1 & 0 \\
a & 1\end{array}\right)$ & $\psi(w, z)=(w+2 a z+b, z+a)$ & $\begin{array}{c}w(n) \sim n^{2} a^{2} \\
z(n) \sim n a .\end{array}$ \\
\hline$\left(\begin{array}{ll}1 & 0 \\
0 & 1\end{array}\right)$ & $\psi(w, z)=(w+b, z)$ & $\begin{array}{c}w(n) \sim n b \\
z(n) \sim z\end{array}$ \\
\hline$\left(\begin{array}{ll}1 & 0 \\
0 & \mu\end{array}\right), \mu \neq 1$ & $\psi(w, z)=(w+b, \mu z)$ & $\begin{array}{c}w(n) \sim n b \\
z(n) \sim \mu^{n} z\end{array}$
\end{tabular}

\section{The parabolic nondiagonalizable case}

Throughout this section, $\varphi$ is a self-map of $\mathbb{B}_{2}$ whose Denjoy-Wolff point is $e_{1}$, which belongs to $\mathcal{D}^{2}\left(e_{1}\right)$ and such that

$$
d \varphi_{e_{1}}=\left(\begin{array}{cc}
1 & 0 \\
\alpha_{1} & 1
\end{array}\right)
$$

with $\alpha_{1} \neq 0$. The linear fractional maps of $\mathbb{B}_{2}$ having the same differential at a boundary fixed point are the generalized Heisenberg translations (see [4]), namely (on $\mathbb{H}_{2}$ ) the maps $(w, z) \mapsto(w+2 \bar{\alpha} z+\beta, z+\alpha)$ with $\Re(\beta) \geq|\alpha|^{2}$. Thus this case will be also called the Heisenberg translation case, and our hope is to find a map $\sigma: \mathbb{B}_{2} \rightarrow \mathbb{C}^{2}$ and a generalized Heisenberg translation $\tau$ such that $\sigma \circ \varphi=\tau \circ \sigma$. This will be the case, up to regularity conditions: 
Theorem 3.1. Let $\varphi \in \mathcal{D}^{5+\varepsilon}\left(e_{1}\right)$. There exists a holomorphic map $\sigma: \mathbb{H}_{2} \rightarrow \mathbb{C}^{2}$ and a generalized Heisenberg translation $\tau:(w, z) \mapsto(w+2 \bar{\alpha} z+\beta, z+\alpha)$ such that:

$$
\sigma \circ \phi=\tau \circ \sigma .
$$

Moreover, there is a domain $\Omega$ contained in a neighbourhood of $\infty$ such that, for any $(w, z) \in \mathbb{H}_{2}, \phi^{[n]}(w, z) \in \Omega$ for $n$ large enough and such that, on $\Omega$, one has for any $\delta \in(0,1 / 2)$ :

$$
\begin{aligned}
\left|\sigma_{1}(w, z)-w\right| & =o\left(|w|^{1 / 2+\delta}\right), \\
\left|\sigma_{2}(w, z)-z\right| & =o\left(|w|^{\delta}\right) .
\end{aligned}
$$

Our first step is to work more on the expansion near infinity of $\phi=$ $U \circ \varphi \circ U^{-1}$.

\subsection{More on the expansion near the boundary point}

We suppose that $\varphi \in \mathcal{D}^{3}\left(e_{1}\right)$. Following the work of Section 2 , since $\gamma_{1}=0$, we know that $\phi$ may be written near $\infty$

$$
\begin{aligned}
& \phi_{1}(w, z)=w+a z+b \frac{z^{3}}{w}+o\left(|w|^{1 / 2}\right), \\
& \phi_{2}(w, z)=z+c+d \frac{z^{2}}{w}+e \frac{z^{4}}{w^{2}}+o(1) .
\end{aligned}
$$

The coefficients $a, b, c, d, e$ are not free.

Lemma 3.2. Under the above definitions and notations, one has $a=2 \bar{c}$ and $b=d=e=0$.

Proof. We first suppose that $\phi$ extends to $\partial \mathbb{H}_{2}$, with the same expansion near $\infty$. Let us write $a=a_{0} e^{i \alpha}, b=b_{0} e^{i \beta}, c=c_{0} e^{i \gamma}, d=d_{0} e^{i \delta}$ and $e=e_{0} e^{i \varepsilon}$. Let $P=\left(t^{2}(1+i \lambda), e^{i \theta} t\right)$ with $\theta, \lambda \in \mathbb{R}$. We know that $\Re\left(\phi_{1}(P)\right) \geq\left|\phi_{2}(P)\right|^{2}$. Writing $\frac{1}{1+i \lambda}=\frac{1}{\sqrt{1+\lambda^{2}}} e^{i g(\lambda)}$ with $\cos (g(\lambda))=\frac{1}{\sqrt{1+\lambda^{2}}}$ and $\sin (g(\lambda))=\frac{-\lambda}{\sqrt{1+\lambda^{2}}}$, the expansion near $\infty$ gives

$$
\begin{aligned}
& \Re\left(\phi_{1}(P)\right)= t^{2}+\left(a_{0} \cos (\alpha+\theta)+\frac{b_{0}}{\sqrt{1+\lambda^{2}}} \cos (\beta+3 \theta+g(\lambda))\right) t+o(t), \\
&\left|\phi_{2}(P)\right|^{2}= t^{2}+2\left(c_{0} \cos (\gamma-\theta)+\frac{d_{0}}{\sqrt{1+\lambda^{2}}} \cos (\delta+\theta+g(\lambda))+\right. \\
&\left.\frac{e_{0}}{1+\lambda^{2}} \cos (\varepsilon+3 \theta+2 g(\lambda))\right) t+o(t) .
\end{aligned}
$$

Fix any $\theta \in \mathbb{R}$ such that $\cos (\alpha+\theta) \neq 0$ and $\cos (\gamma-\theta) \neq 0$. Letting $\lambda \rightarrow \infty$, we get

$$
a_{0} \cos (\alpha+\theta) \geq 2 c_{0} \cos (\gamma-\theta) \text {. }
$$


Since this is true for almost every $\theta$, this means $a=2 c_{0}$ and $\gamma=-\alpha$, namely $a=2 \bar{c}$. Coming back to the inequality $\Re\left(\phi_{1}(P)\right) \geq\left|\phi_{2}(P)\right|^{2}$, this in turn implies

$b_{0} \cos (\beta+3 \theta+g(\lambda)) \geq 2 d_{0} \cos (\delta+\theta+g(\lambda))+\frac{2 e_{0}}{\sqrt{1+\lambda^{2}}} \cos (\varepsilon+3 \theta+2 g(\lambda))$

for any $\theta, \lambda \in \mathbb{R}$. The leading term in $\lambda$ of the left handside of this inequality is $\frac{-b_{0} \lambda}{\sqrt{1+\lambda^{2}}} \sin (\beta+3 \theta)$ whereas at the right handside this is $\frac{-2 d_{0} \lambda}{\sqrt{1+\lambda^{2}}} \sin (\delta+\theta)$. As previously, we obtain $b_{0} \sin (\beta+3 \theta) \leq 2 d_{0} \sin (\delta+\theta)$ for every $\theta \in \mathbb{R}$. This is clearly impossible, unless $b_{0}=d_{0}=0$. This implies also $e_{0}=0$.

For the general case, instead of $P$, we consider $P_{\tau}=\left(t^{2}(1+i \lambda)+\tau, e^{i \theta} t\right)$ with $\tau>0$ and we write $\Re\left(\phi_{1}\left(P_{\tau}\right)\right) \geq\left|\phi_{2}\left(P_{\tau}\right)\right|^{2}$. Letting $\tau \rightarrow 0$, we obtain the same inequality as above and we conclude in a similar way.

Observe that up to conjugation by an automorphism of $\mathbb{H}_{2}$ of the form $(w, z) \mapsto\left(w, e^{i \theta} z\right)$, it is always possible to suppose that $a \in \mathbb{R}^{+}$. Since $a$ is a multiple of $\alpha_{1} \neq 0$, one has in fact $a>0$. We will keep this assumption during all this section.

\subsection{Behavior near infinity, Step 1}

We now suppose that $\varphi$ belongs to $\mathcal{D}^{5+\varepsilon}\left(e_{1}\right)$. Changing slightly the notations and using the results of Lemma 3.2, we may write, near infinity:

$$
\begin{aligned}
\phi_{1}(w, z)=w & +2 a z+b+c \frac{z^{2}}{w}+d \frac{z^{4}}{w^{2}}+e \frac{z^{6}}{w^{3}}+ \\
+ & f \frac{z}{w}+g \frac{z^{3}}{w^{2}}+h \frac{z^{5}}{w^{3}}+r \frac{z^{7}}{w^{4}}+s \frac{z^{9}}{w^{5}}+O\left(\frac{1}{|w|^{1 / 2+\varepsilon / 2}}\right) \\
\phi_{2}(w, z)=z & +a+a^{\prime} \frac{z}{w}+b^{\prime} \frac{z^{3}}{w^{2}}+c^{\prime} \frac{z^{5}}{w^{3}}+d^{\prime} \frac{z^{7}}{w^{4}}+ \\
& +e^{\prime} \frac{1}{w}+f^{\prime} \frac{z^{2}}{w^{2}}+g^{\prime} \frac{z^{4}}{w^{3}}+h^{\prime} \frac{z^{6}}{w^{4}}+r^{\prime} \frac{z^{8}}{w^{5}}+s^{\prime} \frac{z^{10}}{w^{6}}+O\left(\frac{1}{|w|^{3 / 2}}\right)
\end{aligned}
$$

where $a>0$. We could expand one more term of $\phi_{2}$. However this is unnecessary.

Let us introduce some notations. For $(w, z) \in \mathbb{H}_{2},(w(n), z(n))$ means $\phi^{[n]}(w, z)$. For $M, A>0$, the set $K_{M, A}$ is defined by

$$
K_{M, A}=\left\{(w, z) \in \mathbb{H}_{2}: \Re(z) \geq A, \Re(w) \geq M, \frac{|\Im(z)|}{\Re(z)} \leq \frac{1}{4} \text { and } \frac{|\Im(w)|}{\Re(w)} \leq \frac{1}{2}\right\}
$$

In particular, on $K_{M, A}, \Re(w)$ and $|w|$ are comparable. For $M, A$ large enough, $K_{M, A}$ is preserved by $\phi$. This is easy to check for the conditions 
$\Re(w) \geq M$ and $\Re(z) \geq A$. On the other hand, for $M, A$ large enough, one has

$$
\frac{\left|\Im\left(\phi_{2}(w, z)\right)\right|}{\Re\left(\phi_{2}(w, z)\right)} \leq \frac{|\Im(z)|+a / 8}{\Re(z)+7 a / 8} \leq \frac{1}{4} .
$$

In a similar way, there exists a constant $C>0$ such that

$$
\frac{\left|\Im\left(\phi_{1}(w, z)\right)\right|}{\Re\left(\phi_{1}(w, z)\right)} \leq \frac{|\Im(w)|+2 a|\Im(z)|+C}{\Re(w)+2 a \Re(z)-C} \leq \frac{1}{2}
$$

since $2 a\left(\frac{\Re(z)}{2}-|\Im(z)|\right) \geq \frac{a \Re(z)}{2} \geq \frac{3 C}{2}$ provided $A$ is large enough.

Finally, as a matter of notation, for $u, v \in \mathbb{R}^{+}, u \lesssim A_{1}, A_{2}, \ldots v$ means that there exists a positive constant $C$ depending only on $\phi$ and on $A_{1}, A_{2}, \ldots$ such that $u \leq C \times v$. We just write $u \lesssim v$ if $C$ depends only on $\phi$.

Lemma 3.3. Let $K$ be a compact subset of $\mathbb{H}_{2}, M, A>0$. There exists $n_{0} \in \mathbb{N}$ such that, for every $(w, z) \in K,(w(n), z(n)) \in K_{M, A}$ for every $n \geq n_{0}$.

Proof. There exists a neighbourhood $V$ of $\infty$ in $\mathbb{H}_{2}$ and $C>0$ such that, for any $(w, z) \in V$,

$$
\Re\left(\phi_{1}(w, z)\right)-\Re(w)-2 a \Re(z) \geq-C \text { and } \Re\left(\phi_{2}(w, z)\right)-\Re(z) \geq a / 2 .
$$

By the Denjoy-Wolff theorem, there exists a natural number $k_{1}$ such that $k \geq k_{1} \Longrightarrow(w(k), z(k)) \in V$. Now, for $k \geq k_{1}$, one has

$$
\Re(z(k)) \geq \min _{\left(w^{\prime}, z^{\prime}\right) \in K} \Re\left(z^{\prime}\left(k_{1}\right)\right)+\left(k-k_{1}\right) a / 2 \geq \max (A, C / a)
$$

for $k \geq k_{2}$. In the same vein, for $k \geq k_{2}$, we get

$$
\Re(w(k)) \geq \min _{\left(w^{\prime}, z^{\prime}\right) \in K} \Re\left(w^{\prime}\left(k_{2}\right)\right)+\left(k-k_{2}\right) C \geq M
$$

for $k$ large enough. On the other hand, we have eventually $|\Im(z(k+1))| \leq$ $\mid \Im\left(z(k) \mid+\frac{a}{10}\right.$. Combining this with the inequality above, we get $\frac{|\Im(z(k))|}{\Re(z(k))} \leq \frac{1}{4}$ as soon as $k$ is large enough. The proof of the last inequality follows exactly the same lines.

Our method to construct the map $\sigma$ is based on a very careful analysis of the behavior of $(w(n), z(n))$ followed by a suitable normalization. The previous lemma allows us to restrict the study to the case where $(w, z)$ belongs to $K_{M, A}$ with $M$ and $A$ as large as necessary. We obtain a first estimate on the behavior of $(w(n), z(n))$ thanks to the asymptotic expansion of $\phi$. Next, if we introduce this estimate into the expansion, then we get a better estimate. We will repeat the process as many times as necessary. Moreover, we have to keep precise estimations to ensure that $\sigma$ is holomorphic, nonzero, and satisfies some inequalities near infinity. We begin with the first step. 
Lemma 3.4. For $M, A$ large enough, for any $(w, z) \in K_{M, A}$, the following estimates hold true:

$$
\begin{aligned}
& |z|+n \lesssim|z(n)| \lesssim|z|+n \\
& \Re(w)+n^{2} \lesssim \Re(w(n)) \lesssim \Re(w)+n^{2} .
\end{aligned}
$$

Proof. For $M, A$ large enough, $K_{M, A}$ is stable by $\phi$ and one has, for any $(w, z) \in K_{M, A}:$

$$
\begin{gathered}
\left|\Re\left(\phi_{1}(w, z)\right)-\Re(w)-2 a \Re(z)\right| \leq 1, \\
\left|\phi_{2}(w, z)-(z+a)\right| \leq a / 2 .
\end{gathered}
$$

In particular, the second inequality gives $|z(n)-n a-z| \leq \frac{n a}{2}$, which in turn implies the first assertion of the lemma. For the second one, observe that

$$
|\Re(w(n))-\Re(w)-2 a \Re(z(0)+\cdots+z(n-1))| \leq n .
$$

Using $\frac{a k}{2}+\Re(z) \leq \Re(z(k)) \leq \frac{3 a k}{2}+\Re(z)$, we get the result.

Proposition 3.5. For $M, A$ large enough, for any $(w, z)$ in $K_{M, A}$ one has:

(a) $|z(n)-n a| \lesssim \log (n+1)+|z|$,

(b) $\left|w(n)-n(n-1) a^{2}\right| \lesssim n \log (n+1)+n|z|+|w|$.

\section{Proof.}

a) Let us write $z(n)=n a+z^{\prime}(n)$. Using the expansion of $\phi$ and Lemma 3.4, we get:

$$
\begin{aligned}
\left|z^{\prime}(n+1)-z^{\prime}(n)\right| & \lesssim \frac{|z(n)|}{|w(n)|}+\frac{\left|z^{3}(n)\right|}{\left|w^{2}(n)\right|}+\frac{\left|z^{5}(n)\right|}{\left|w^{3}(n)\right|}+\frac{\left|z^{7}(n)\right|}{\left|w^{4}(n)\right|}+\frac{1}{|w(n)|} \\
& \lesssim \frac{1}{|w(n)|^{1 / 2}} \\
& \lesssim \frac{1}{n+1} .
\end{aligned}
$$

Summing the differences $z^{\prime}(n+1)-z^{\prime}(n)$ and using $z^{\prime}(0)=z$, we obtain assertion a).

b) We write now $w(n)=n(n-1) a^{2}+w^{\prime}(n)$. The expansion gives:

$$
\begin{aligned}
\left|w^{\prime}(n+1)-w^{\prime}(n)\right| & \lesssim|z(n)-n a|+1 \\
& \lesssim \log (n+1)+|z|+1 .
\end{aligned}
$$

We conclude as above.

In particular, the result of Proposition 3.5 shows that for the parabolic non-diagonalizable case the orbits of $\phi$ mimic very well the orbits of the corresponding linear fractional map. It is interesting to compare this property with the example of Section 4.6. 


\subsection{Behavior near infinity, Step 2}

To go further in the expansion of $w(n)$ and $z(n)$, we need to have an estimation of the following terms in the expansion of $\phi$. This will be done thanks to the results of Step 1 . We begin with the terms coming from the expansion of $\phi_{2}$ :

Lemma 3.6. For $M, A$ large enough, for any $\delta \in(0,1)$, for any $(w, z) \in$ $K_{M, A}$, for any $n \geq 1$, one has

$$
\left|\frac{z(n)}{w(n)}-\frac{1}{a n}\right| \lesssim \frac{\log (n+1)}{n^{2}}+\frac{|w|^{\delta}}{(n+1)^{1+2 \delta}} .
$$

The same holds true for $\left|\frac{z^{3}(n)}{w^{2}(n)}-\frac{1}{a n}\right|,\left|\frac{z^{5}(n)}{w^{3}(n)}-\frac{1}{a n}\right|,\left|\frac{z^{7}(n)}{w^{4}(n)}-\frac{1}{a n}\right|$.

Proof. Since the proofs are similar, we just proceed with the last term. We write

$$
\frac{z^{7}(n)}{w^{4}(n)}-\frac{1}{a n}=\frac{a n z^{7}(n)-w^{4}(n)}{a n w^{4}(n)}=\frac{a n\left(z^{7}(n)-(a n)^{7}\right)-\left(w^{4}(n)-(a n)^{8}\right)}{a n w^{4}(n)} .
$$

Using successively the mean value theorem, Lemma 3.4 and Proposition 3.5, we have:

$$
\begin{aligned}
\left|z^{7}(n)-(a n)^{7}\right| & \leq 7 \max \left(|z(n)|^{6},(a n)^{6}\right)|z(n)-a n| \\
& \lesssim(\log (n+1)+|z|)(n+|z|)^{6} .
\end{aligned}
$$

In a similar way, one gets

$$
\left|w^{4}(n)-(a n)^{8}\right| \lesssim(n \log (n+1)+n|z|+|w|)\left(n^{2}+n|z|+|w|\right)^{3} .
$$

On the other hand, one has:

$$
(n+|z|)^{6} \leq\left(n+|w|^{1 / 2}\right)^{6} \lesssim\left(n^{2}+|w|\right)^{3}
$$

and

$$
\left(n^{2}+n|z|+|w|\right)^{3} \lesssim\left(n^{2}+|w|\right)^{3} .
$$

Using $|w(n)| \geq \Re(w(n)) \gtrsim n^{2}+\Re(w) \geq n^{2}+|w|$ on $K_{M, A}$, we get:

$$
\left|\frac{z^{7}(n)}{w^{4}(n)}-\frac{1}{a n}\right| \lesssim \frac{n \log (n+1)+n|w|^{1 / 2}+|w|}{n\left(n^{2}+|w|\right)} .
$$

To conclude, it suffices to observe that

$$
\frac{|w|^{1 / 2}}{n^{2}+|w|} \leq \frac{|w|^{\delta}}{n^{2 \delta}} \text { and } \frac{|w|}{n\left(n^{2}+|w|\right)} \leq \frac{|w|^{\delta}}{n^{1+2 \delta}} .
$$


Lemma 3.7. For $M, A$ large enough, for any $\delta \in(0,1)$, for any $(w, z) \in$ $K_{M, A}$, for any $n \geq 1$, one has:

$$
\left|\frac{z^{2}(n)}{w(n)}-1\right| \lesssim \frac{\log (n+1)}{n}+\frac{|w|^{1 / 2}}{n}+\frac{|w|}{n^{2}}
$$

The same holds true for $\left|\frac{z^{4}(n)}{w^{2}(n)}-1\right|$ and $\left|\frac{z^{6}(n)}{w^{3}(n)}-1\right|$.

We omit the proof which is very similar (see also Lemma 3.11 below). We are ready to obtain two other terms in $w(n)$ and $z(n)$.

Proposition 3.8. For $M, A$ large enough, there exists a holomorphic function $D(w, z)$ on $K_{M, A}$ such that, for any $(w, z)$ in $K_{M, A}$ :

(a) $\left|z(n)-n a-\frac{\left(a^{\prime}+b^{\prime}+c^{\prime}+d^{\prime}\right)}{a} \log (n+1)-D(w, z)\right| \lesssim \frac{\log (n+1)}{n}+\frac{|w|^{1 / 2}}{n}$,

and for any $\delta \in(0,1),|D(w, z)-z| \lesssim_{\delta}|w|^{\delta}$.

(b) $\mid w(n)-n(n-1) a^{2}-2\left(a^{\prime}+b^{\prime}+c^{\prime}+d^{\prime}\right) n \log (n+1)-(2 a D(w, z)$

$\left.-2\left(a^{\prime}+b^{\prime}+c^{\prime}+d^{\prime}\right)+(b+c+d+e)\right)\left.n|\lesssim| w\right|^{1 / 2} \log (n+1)+\log ^{2}(n+1)+|w|$.

Proof. (a) Let us write $z(n)=n a+\frac{\left(a^{\prime}+b^{\prime}+c^{\prime}+d^{\prime}\right)}{a} \log (n+1)+z^{\prime}(n)$. The expansion of $\phi_{2}$ gives

$$
\begin{aligned}
z^{\prime}(n+1)-z^{\prime}(n)= & a^{\prime}\left(\frac{z(n)}{w(n)}-\frac{1}{a n}\right)+b^{\prime}\left(\frac{z^{3}(n)}{w^{2}(n)}-\frac{1}{a n}\right) \\
& +c^{\prime}\left(\frac{z^{5}(n)}{w^{3}(n)}-\frac{1}{a n}\right)+d^{\prime}\left(\frac{z^{7}(n)}{w^{4}(n)}-\frac{1}{a n}\right)+\frac{\varepsilon_{n}(w, z)}{n^{2}},
\end{aligned}
$$

where $\varepsilon_{n}$ is bounded on $K_{M, A}$ independently of $n$. Using the result of Lemma 3.6, we have

$$
\sum_{n \geq 0}\left|z^{\prime}(n+1)-z^{\prime}(n)\right| \lesssim_{\delta}|w|^{\delta}
$$

Hence the sequence $\left(z^{\prime}(n)\right)$ converges uniformly on $K_{M, A}$ to a holomorphic function $D$. Moreover, since $z^{\prime}(0)=z$ the previous inequality becomes $|D(w, z)-z| \lesssim_{\delta}|w|^{\delta}$. Let us write now

$$
z(n)=n a+\frac{\left(a^{\prime}+b^{\prime}+c^{\prime}+d^{\prime}\right)}{a} \log (n+1)+D(w, z)+z^{\prime \prime}(n)
$$

where $z^{\prime \prime}(n)$ goes to 0 as $n$ goes to infinity. 
Applying Lemma 3.6 with $\delta=1 / 2$, we have

$$
\left|z^{\prime \prime}(n+1)-z^{\prime \prime}(n)\right| \lesssim \frac{\log (n+1)}{n^{2}}+\frac{|w|^{1 / 2}}{n^{2}}
$$

Finally this gives

$$
\left|z^{\prime \prime}(n)\right| \leq \sum_{k \geq n}\left|z^{\prime \prime}(k+1)-z^{\prime \prime}(k)\right| \lesssim \frac{\log (n+1)}{n}+\frac{|w|^{1 / 2}}{n}
$$

as desired. Concerning (b), we write

$$
\begin{aligned}
w(n)= & n(n-1) a^{2}+2\left(a^{\prime}+b^{\prime}+c^{\prime}+d^{\prime}\right) n \log (n+1) \\
& \left.+\left(2 a D(w, z)-2\left(a^{\prime}+b^{\prime}+c^{\prime}+d^{\prime}\right)+b+c+d+e\right)\right) n+w^{\prime}(n) .
\end{aligned}
$$

We have

$$
\begin{aligned}
w^{\prime}(n+1)-w^{\prime}(n)= & 2 a\left(z(n)-n a-\frac{\left(a^{\prime}+b^{\prime}+c^{\prime}+d^{\prime}\right)}{a} \log (n+1)-D(w, z)\right) \\
& +c\left(\frac{z^{2}(n)}{w(n)}-1\right)+d\left(\frac{z^{4}(n)}{w^{2}(n)}-1\right)+e\left(\frac{z^{6}(n)}{w^{3}(n)}-1\right) .
\end{aligned}
$$

Lemma 3.7 and the first part of the proof of this proposition ensures that

$$
\left|w^{\prime}(n+1)-w^{\prime}(n)\right| \lesssim \frac{\log (n+1)}{n}+\frac{|w|^{1 / 2}}{n}+\frac{|w|}{n^{2}}
$$

The result of Proposition 3.8 follows now from a summation as before.

\subsection{Behavior near infinity, Step 3}

We have almost done the work for $z(n)$. However, we need to have a constant term in $w(n)$ and this requires to go further in the expansion of $z(n)$. To this aim, we need to have an estimation of the remaining terms coming from the expansion of $\phi_{2}$ and also to improve the content of Lemmas 3.6 and 3.7.

Lemma 3.9. For $M, A$ large enough, for any $\delta \in(0,1)$, for any $j \geq 0$, one has:

$$
\begin{gathered}
\left|\frac{z^{2 j}(n)}{w^{1+j}(n)}-\frac{1}{a^{2} n^{2}}\right| \lesssim_{j} \frac{\log (n+1)}{n^{3}}+\frac{|w|^{\delta}}{n^{2+2 \delta}}, \\
\left|\frac{1}{w^{1+j}(n)}-\frac{1}{\left(a^{2} n^{2}\right)^{1+j}}\right| \lesssim_{j} \frac{\log (n+1)}{n^{2+2 j+1}}+\frac{|w|^{\delta}}{n^{2+2 j+2 \delta}} .
\end{gathered}
$$


Proof. Using successively the mean value theorem and Proposition 3.5, one has:

$$
\begin{aligned}
\mid \frac{z^{2 j}(n)}{w^{1+j}(n)}- & \frac{1}{a^{2} n^{2}}\left|\lesssim \frac{1}{n^{2}}\right| \frac{n^{2} a^{2}\left(z^{2 j}(n)-\left(a^{2} n^{2}\right)^{2 j}\right)-\left(w^{1+j}(n)-\left(a^{2} n^{2}\right)^{1+j}\right)}{w^{1+j}(n)} \mid \\
\lesssim & \lesssim_{j} \frac{1}{n^{2}\left(n^{2}+|w|\right)^{1+j}}\left(n^{2}\left(n+|w|^{1 / 2}\right)^{2 j-1}\left(\log (n+1)+|w|^{1 / 2}\right)+\right. \\
& \left.\quad+\left(n \log (n+1)+n|w|^{1 / 2}+|w|\right)\left(n^{2}+n|w|^{1 / 2}+|w|\right)^{j}\right) \\
\lesssim & \frac{\left(n \log (n+1)+n|w|^{1 / 2}+|w|\right)\left(n^{2}+|w|\right)^{j}}{n^{2}\left(n^{2}+|w|\right)^{1+j}} \\
\lesssim & \frac{n \log (n+1)+n|w|^{1 / 2}+|w|}{n^{2}\left(n^{2}+|w|\right)} .
\end{aligned}
$$

We conclude as in Lemma 3.6. The proof for $\left|\frac{1}{w^{1+j}(n)}-\frac{1}{\left(a^{2} n^{2}\right)^{1+j}}\right|$ is the same.

Lemma 3.10. Let $j \geq 0$. There exist two complex numbers $\theta_{1}$ and $\theta_{2}$ such that, for $M, A$ large enough, for any $\delta \in(0,1 / 2)$, for any $(w, z) \in K_{M, A}$, for any $n \geq 1$, one has:

$\left|\frac{z^{1+2 j}(n)}{w^{1+j}(n)}-\frac{1}{a n}-\frac{\theta_{1} \log (n+1)}{n^{2}}-\frac{\theta_{2}}{n^{2}}+\frac{D(w, z)}{a^{2} n^{2}}\right| \lesssim \delta, j \frac{\log ^{2 j+1}(n+1)|w|^{1 / 2+\delta}}{n^{2+2 \delta}}$.

Proof. For the sake of clarity, we set:

$$
\begin{aligned}
Z(n)= & n a+ \\
W(n)= & n(n-1) a^{2}+2\left(a^{\prime}+b^{\prime}+c^{\prime}+d^{\prime}\right) n \log (n+1)+ \\
& \quad+\left(2 a D(w, z)-2\left(a^{\prime}+b^{\prime}+c^{\prime}+d^{\prime}\right)+(b+c+d+e)\right) n .
\end{aligned}
$$

We argue as in Lemma 3.6, replacing Proposition 3.5 by the better Proposition 3.8. Thus, we write:

$$
\begin{gathered}
\frac{z^{1+2 j}(n)}{w^{1+j}(n)}-\frac{1}{a n}=\frac{1}{a n w^{1+j}(n)}\left(\operatorname{an}\left(z^{1+2 j}(n)-Z^{1+2 j}(n)\right)-\left(w^{1+j}(n)-W^{1+j}(n)\right)\right) \\
+\frac{1}{a n w^{1+j}(n)}\left(a n Z^{1+2 j}(n)-W^{1+j}(n)\right) .
\end{gathered}
$$

On the one hand, Proposition 3.8 and the mean value theorem give:

$$
\frac{\left|w^{1+j}(n)-W^{1+j}(n)\right|}{a n w^{1+j}(n)} \lesssim_{j} \frac{\left(|w|^{1 / 2} \log (n+1)+\log ^{2}(n+1)+|w|\right)\left(n^{2}+|w|\right)^{j}}{n\left(n^{2}+|w|\right)^{1+j}} .
$$


If we observe that

$$
\frac{|w|^{1 / 2} \log (n+1)}{n \cdot\left(n^{2}+|w|\right)} \leq \frac{|w|^{1 / 2-\delta}}{|w|^{1 / 2-\delta}} \times \frac{|w|^{\delta} \log (n+1)}{n n^{2(1 / 2+\delta)}}
$$

and that

$$
\frac{|w|}{n\left(n^{2}+|w|\right)} \leq \frac{|w|^{1 / 2-\delta}}{|w|^{1 / 2-\delta}} \times \frac{|w|^{1 / 2+\delta}}{n n^{2(1 / 2+\delta)}}
$$

then we get the appropriate estimates for this part of the sum. The same reasoning applies for $n\left|z^{1+2 j}(n)-Z^{1+2 j}(n)\right|$. It remains to consider $n a Z^{1+2 j}(n)-$ $W^{1+j}(n)$. We expand the products and we remark that the terms in $n^{2+2 j}$ vanish. We then find

$n^{1+2 j}\left(\mu_{1} \log (n+1)+\mu_{2}-a^{2 j+1} D(w, z)\right)+\sum_{k \leq 2 j-1} n^{k+1} \sum_{l+m \leq 2 j+1-k} \theta_{k, l, m} \log ^{l}(n+1) D(w, z)^{m}$

where the $\mu_{i}$ 's and the $\theta_{k, l, m}$ 's are complex numbers. Furthermore, we have:

$$
\begin{aligned}
\left.\mid \frac{n^{1+2 j}\left(\mu_{1} \log (n+1)+\right.}{n a w^{1+j}(n)}-a^{2 j+1} D(w, z)\right) & -\frac{\mu_{1} \log (n+1)+\mu_{2}-a^{2 j+1} D(w, z)}{a^{2 j+3} n^{2}} \mid \\
& \lesssim_{j}\left(\log (n+1)+|w|^{\delta}\right)\left|\frac{n^{1+2 j}}{n a w^{1+j}(n)}-\frac{1}{a^{2 j+3} n^{2}}\right| \\
& \lesssim_{j}\left(\log (n+1)+|w|^{\delta}\right) n^{2 j}\left|\frac{1}{w^{1+j}(n)}-\frac{1}{\left(a^{2} n^{2}\right)^{1+j}}\right| \\
& \lesssim_{j}\left(\log (n+1)+|w|^{\delta}\right) \times\left(\frac{\log (n+1)}{n^{3}}+\frac{|w|^{\delta}}{n^{2+2 \delta}}\right) .
\end{aligned}
$$

This gives also a correct majoration, since $2 \delta \leq 1 / 2+\delta$ for $\delta \in(0,1 / 2)$. To conclude, we study the last term: for $k \leq 2 j-1, l, m \geq 0$ with $l+m \leq$ $2 j-1+k$ and $m \geq 1$, one has:

$$
\begin{aligned}
\frac{\left|n^{k+1} \log ^{l}(n+1) D^{m}(w, z)\right|}{\left|n a w^{1+j}(n)\right|} & \lesssim \frac{n^{k+1} \log ^{l}(n+1)|w|^{m / 2}}{n\left(n^{2}+|w|\right)^{1+j}} \\
& \lesssim \frac{\log ^{l}(n+1)|w|^{m / 2}}{\left(n^{2}+|w|\right)^{1+j-k / 2}} \\
& \lesssim \frac{\log ^{l}(n+1)|w|^{1 / 2+\delta}}{n^{2(1+j-k / 2-(m-1) / 2+\delta)}}
\end{aligned}
$$

Now, $j-(m-1) / 2-k / 2 \geq l / 2 \geq 0$, and $l \leq 2 j+1$, which finally implies

$$
\frac{\left|n^{k+1} \log ^{l}(n+1) D^{m}(w, z)\right|}{\left|n a w^{1+j}(n)\right|} \lesssim_{\delta, j} \frac{\log ^{2 j+1}(n+1) \cdot|w|^{1 / 2+\delta}}{n^{2+2 \delta}} .
$$

If $m=0$, then the previous inequality holds trivially true, using $k \leq 2 j-1$. 
Lemma 3.11. Let $j \geq 1$. There exists a complex number $\theta_{1}$ such that, for any $\delta \in(0,1 / 2)$, for any $M, A$ large enough, for any $(w, z) \in K_{M, A}$ :

$$
\left|\frac{z^{2 j}(n)}{w^{j}(n)}-1-\frac{\theta_{1}}{n}\right| \lesssim_{\delta, j} \frac{|w|^{1+2 \delta} \log ^{2 j}(n+1)}{n^{1+2 \delta}} .
$$

Proof. As above, we write

$$
\frac{z^{2 j}(n)}{w^{j}(n)}-1=\frac{\left(z^{2 j}(n)-Z^{2 j}(n)\right)-\left(w^{j}(n)-W^{j}(n)\right)}{w^{j}(n)}+\frac{Z^{2 j}(n)-W^{j}(n)}{w^{j}(n)} .
$$

Our (now classical!) argument shows that

$$
\frac{\left|w^{j}(n)-W^{j}(n)\right|}{\left|w^{j}(n)\right|}, \frac{\left|z^{2 j}(n)-Z^{2 j}(n)\right|}{\left|w^{j}(n)\right|} \lesssim \delta, j \frac{\log ^{2}(n+1)}{n^{2}}+\frac{|w|^{1 / 2+\delta}}{n^{1+2 \delta}} .
$$

Moreover, when we compute $Z^{2 j}(n)-W^{j}(n)$, the terms in $n^{2 j}, n^{2 j-1} \log (n+1)$ and $D(w, z) n^{2 j-1}$ simplifies. We obtain a sum like

$$
\mu_{1} n^{2 j-1}+\sum_{k \leq 2 j-2} n^{k} \sum_{l+m \leq 2 j-k} \theta_{k, l, m} \log ^{l}(n+1) D(w, z)^{m} .
$$

We proceed as above to show the existence of $\theta_{1}$ such that

$$
\left|\frac{Z^{2 j}(n)-W^{j}(n)}{w^{j}(n)}-\frac{\theta_{1}}{n}\right| \lesssim \delta, j \frac{\log ^{2 j}(n+1)|w|^{1 / 2+\delta}}{n^{1+2 \delta}} .
$$

It is time to build the second component of $\sigma$.

Proposition 3.12. For $M, A$ large enough, there exists a holomorphic function $E(w, z)$ on $K_{M, A}$ and complex numbers $\theta_{1}, \theta_{2}, \theta_{3}, \theta_{4}, \theta_{5}$ with $\theta_{3}=$ $\frac{a^{\prime}+b^{\prime}+c^{\prime}+d^{\prime}}{a^{2}}$ such that, for any $(w, z)$ in $K_{M, A}$, for any $\delta \in(0,1 / 2)$, one has:

(a) $\mid z(n)-n a-\frac{\left(a^{\prime}+b^{\prime}+c^{\prime}+d^{\prime}\right)}{a} \log (n+1)-D(w, z)-\frac{\theta_{1} \log (n+1)}{n}$

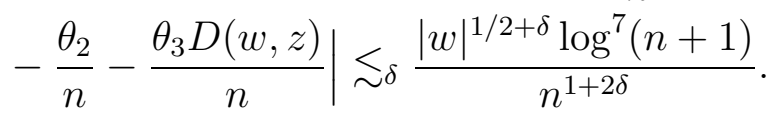

(b) $w(n)-n(n-1) a^{2}-2\left(a^{\prime}+b^{\prime}+c^{\prime}+d^{\prime}\right) n \log (n+1)-(2 a D(w, z)$

$$
\begin{aligned}
& \left.-2\left(a^{\prime}+b^{\prime}+c^{\prime}+d^{\prime}\right)+(b+c+d+e)\right) n-\theta_{4} \log ^{2}(n+1) \\
& -\theta_{5} \log (n+1)-2 a \theta_{3} D(w, z) \log (n+1)-E(w, z)=o(1),
\end{aligned}
$$

with $|E(w, z)-w| \lesssim \delta|w|^{1 / 2+\delta}$. 
Proof. (a) As usual, we write

$$
z(n)=n a+\left(\frac{a^{\prime}+b^{\prime}+c^{\prime}+d^{\prime}}{a}\right) \log (n+1)+D(w, z)+z^{\prime}(n)
$$

and we use the expansion of $\phi_{2}$ to estimate $z^{\prime}(n+1)-z^{\prime}(n)$ :

$$
\begin{aligned}
z^{\prime}(n+1)-z^{\prime}(n)= & a^{\prime}\left(\frac{z(n)}{w(n)}-\frac{1}{a n}\right)+b^{\prime}\left(\frac{z^{3}(n)}{w^{2}(n)}-\frac{1}{a n}\right)+c^{\prime}\left(\frac{z^{5}(n)}{w^{3}(n)}-\frac{1}{a n}\right) \\
& +d^{\prime}\left(\frac{z^{7}(n)}{w^{4}(n)}-\frac{1}{a n}\right)+\frac{e^{\prime}}{w(n)}+f^{\prime} \frac{z^{2}(n)}{w^{2}(n)}+g^{\prime} \frac{z^{4}(n)}{w^{3}(n)} \\
& +h^{\prime} \frac{z^{6}(n)}{w^{4}(n)}+r^{\prime} \frac{z^{8}(n)}{w^{5}(n)}+s^{\prime} \frac{z^{10}(n)}{w^{6}(n)}+\frac{M_{n}(w, z)}{n^{3}}
\end{aligned}
$$

where $M_{n}$ is bounded (independently of $n$ ) on $K_{M, A}$. Fix $\delta \in(\delta, 1 / 2)$. Using Lemma 3.9 and Lemma 3.10, we find complex numbers $\mu_{1}, \mu_{2}$ such that:

$$
\begin{array}{r}
\left|z^{\prime}(n+1)-z^{\prime}(n)-\frac{\mu_{1} \log (n+1)}{n^{2}}-\frac{\mu_{2}}{n^{2}}+\frac{a^{\prime}+b^{\prime}+c^{\prime}+d^{\prime}}{a^{2} n^{2}} D(w, z)\right| \\
\lesssim_{\delta} \frac{|w|^{1 / 2+\delta} \log ^{7}(n+1)}{n^{2+2 \delta}} .
\end{array}
$$

This gives (a) by a summation and the triangle inequality. The sum is now from $n$ to $+\infty$ (this explains the sign of $\theta_{3}$ ) and we use the fact that $z^{\prime}(n)$ tends to 0 as $n$ goes to $+\infty$.

(b) We write now, with the notations of Lemma 3.10, $w(n)=W(n)+w^{\prime}(n)$. The expansion of $\phi_{1}$ near infinity implies:

$$
\begin{aligned}
w^{\prime}(n+1)-w^{\prime}(n)=- & 2 n a^{2}-2\left(a^{\prime}+b^{\prime}+c^{\prime}+d^{\prime}\right) \log (n+1)-2 a D(w, z) \\
& -(b+c+d+e)+2 a z(n)+b+c \frac{z^{2}(n)}{w(n)}+d \frac{z^{4}(n)}{w^{2}(n)} \\
& +e \frac{z^{6}(n)}{w^{3}(n)}+f \frac{z(n)}{w(n)}+g \frac{z^{3}(n)}{w^{2}(n)}+h \frac{z^{5}(n)}{w^{3}(n)}+r \frac{z^{7}(n)}{w^{4}(n)} \\
& +s \frac{z^{9}(n)}{w^{5}(n)}+\frac{t}{n}+\frac{M_{1, n}(w, z)}{n^{1+\varepsilon}},
\end{aligned}
$$

the function $M_{1, n}$ being bounded (independently on $n$ ) on $K_{M, A}$. We replace $2 a z(n)$ by the expression obtained in part (a) of this proposition, the rest being $M_{2, n}(w, z)$ with

$$
\left|M_{2, n}(w, z)\right| \lesssim \delta \frac{|w|^{1 / 2+\delta} \log ^{7}(n+1)}{n^{1+2 \delta}} .
$$


Next, we replace $\frac{z^{2}(n)}{w(n)}$ by $1+\frac{\gamma_{1}}{n}+M_{3, n}(w, z)$ with

$$
\left|M_{3, n}(w, z)\right| \lesssim_{\delta} \frac{|w|^{1 / 2+\delta} \log ^{2}(n+1)}{n^{1+2 \delta}} .
$$

We do the same for $\frac{z^{4}(n)}{w^{2}(n)} \frac{z^{6}(n)}{w^{3}(n)}$ and we replace $\frac{z(n)}{w(n)}$ by $\frac{1}{a n}+M_{4, n}(w, z)$ with

$$
\left|M_{4, n}(w, z)\right| \lesssim_{\delta} \frac{|w|^{\delta}}{n^{1+2 \delta}}+\frac{\log (n+1)}{n^{2}}
$$

(the same for $\left.\frac{z^{3}(n)}{w^{2}(n)}, \ldots, \frac{z^{9}(n)}{w^{10}(n)}\right)$. We finally find:

$$
\begin{array}{r}
\left|w^{\prime}(n+1)-w^{\prime}(n)-\theta_{4} \frac{\log (n+1)}{n}-\frac{\theta_{5}}{n}-2 a \theta_{3} \frac{D(w, z)}{n}\right| \\
\quad \delta_{\delta} \frac{|w|^{1 / 2+\delta} \log ^{7}(n+1)}{n^{1+2 \delta}}+\frac{1}{n^{1+\varepsilon}} .
\end{array}
$$

Hence, $w^{\prime}(n)-\theta_{4} \log ^{2}(n+1)-\theta_{5} \log (n+1)-2 a \theta_{3} D(w, z) \log (n+1)$ converges uniformly on $K_{M, A}$ to a holomorphic function $E$. Moreover (3.3) and $w^{\prime}(0)=w$ ensure that

$$
|E(w, z)-w| \lesssim_{\delta}|w|^{1 / 2+\delta}
$$

In the previous proposition, the precise value of $\theta_{1}, \theta_{2}, \theta_{4}$ and $\theta_{5}$ can be computed but is unimportant. On the contrary, the value of $\theta_{3}$ is crucial for the simplification which appears in the forthcoming argument.

\subsection{Proof of Theorem 3.1}

Our candidate for the function $\sigma$ is $\sigma=(E, D)$ which is defined only on $K_{M, A}$ (but satisfies the appropriate estimates of Theorem 3.1 on $\Omega=K_{M, A}$ ). Take now $K$ any compact subset of $\mathbb{H}_{2}$. There exists an integer $n_{0}$ such that, for $n \geq n_{0}$, the sequence $(w(n), z(n))$ belongs to $K_{M, A}$. Hence, the whole estimates given by the previous lemmas and propositions remain true if we suppose everywhere $n \geq n_{0}$ and if we replace $(w, z)$ by $\left(w\left(n_{0}\right), z\left(n_{0}\right)\right)$. In particular, we may extend the definitions of $E$ and $D$ to $K$ where they are still holomorphic and satisfy

$$
\begin{aligned}
D(w, z)= & \lim _{n \rightarrow+\infty}\left(z(n)-n a-\frac{a^{\prime}+b^{\prime}+c^{\prime}+d}{a} \log (n+1)\right) \\
E(w, z)= & \lim _{n \rightarrow+\infty}\left(w(n)-n(n-1) a^{2}-2\left(a^{\prime}+b^{\prime}+c^{\prime}+d^{\prime}\right) n \log (n+1)\right. \\
& -\left(2 a D(w, z)-2\left(a^{\prime}+b^{\prime}+c^{\prime}+d^{\prime}\right)+(b+c+d+e)\right) n-\theta_{4} \log ^{2}(n+1) \\
& \left.-\theta_{5} \log (n+1)-2 \frac{\left(a^{\prime}+b^{\prime}+c^{\prime}+d^{\prime}\right)}{a} D(w, z) \log (n+1)\right)
\end{aligned}
$$


(the only thing that we do not keep is the asymptotic evaluation of $D$ and $E$, because now we just consider sums between $n_{0}$ and $+\infty$ and $\left.w^{\prime}\left(n_{0}\right) \neq w\right)$. We claim that $\sigma \circ \phi=\tau \circ \sigma$ for $\tau$ an appropriate generalized Heisenberg translation. Indeed, one has:

$$
\begin{aligned}
D \circ \phi(w, z) & =\lim _{n \rightarrow+\infty}\left(z(n+1)-n a-\frac{a^{\prime}+b^{\prime}+c^{\prime}+d^{\prime}}{a} \log (n+1)\right) \\
& =\lim _{n \rightarrow+\infty}\left(z(n+1)-(n+1) a-\frac{a^{\prime}+b^{\prime}+c^{\prime}+d^{\prime}}{a} \log (n+2)\right)+a \\
& =D(w, z)+a .
\end{aligned}
$$

Using this, we also have

$$
\begin{aligned}
E \circ \phi & (w, z)= \\
= & \lim _{n \rightarrow+\infty}\left(w(n+1)-n(n-1) a^{2}-2\left(a^{\prime}+b^{\prime}+c^{\prime}+d^{\prime}\right) n \log (n+1)\right. \\
& -\left(2 a D \circ \phi(w, z)-2\left(a^{\prime}+b^{\prime}+c^{\prime}+d^{\prime}\right)+(b+c+d+e)\right) n \\
& \left.-\theta_{4} \log ^{2}(n+1)-\theta_{5} \log (n+1)-2 \frac{a^{\prime}+b^{\prime}+c^{\prime}+d^{\prime}}{a} D \circ \phi(w, z) \log (n+1)\right) \\
= & \lim _{n \rightarrow+\infty}\left(w(n+1)-n(n+1) a^{2}-2\left(a^{\prime}+b^{\prime}+c^{\prime}+d^{\prime}\right)(n+1) \log (n+2)\right. \\
& -\left(2 a D(w, z)-2\left(a^{\prime}+b^{\prime}+c^{\prime}+d^{\prime}\right)+(b+c+d+e)\right)(n+1) \\
& -\theta_{4} \log ^{2}(n+2)-\theta_{5} \log (n+2)-2 \frac{a^{\prime}+b^{\prime}+c^{\prime}+d^{\prime}}{a} D(w, z) \log (n+2) \\
& +2\left(a^{\prime}+b^{\prime}+c^{\prime}+d^{\prime}\right) \log (n+1)+2\left(a^{\prime}+b^{\prime}+c^{\prime}+d^{\prime}\right)+2 a D(w, z) \\
& \left.+2\left(a^{\prime}+b^{\prime}+c^{\prime}+d^{\prime}\right)+(b+c+d+e)-2\left(a^{\prime}+b^{\prime}+c^{\prime}+d^{\prime}\right) \log (n+1)\right) \\
= & E(w, z)+2 a D(w, z)+(b+c+d+e) .
\end{aligned}
$$

Thus, $\sigma \circ \phi=\tau \circ \sigma$ with $\tau(w, z)=(w+2 a z+(b+c+d+e), z+a)$.

Remark 3.13. Taking for instance $\delta=1 / 4$ in Theorem 3.1, we have for any $(w, z) \in \mathbb{H}_{2}$ :

$$
\left|D \circ \phi^{[n]}(w, z)-\phi_{2}^{[n]}(w, z)\right|=o\left(\left|\phi_{1}^{[n]}(w, z)\right|^{1 / 4}\right) .
$$

Using the asymptotic behavior of $\phi_{1}^{[n]}(w, z)$ and $\phi_{2}^{[n]}(w, z)$, this means that we have

$$
\left|D \circ \phi^{[n]}(w, z)-\phi_{2}^{[n]}(w, z)\right|=o\left(\phi_{2}^{[n]}(w, z)\right)
$$

which corresponds to the estimations that we will have in the hyperbolic case. 
Remark 3.14. We may observe that the linearization of the first and of the second coordinates have to be done simultaneously. This will not be the case for the hyperbolic maps, where the study of the first coordinate will be considerably easier.

Remark 3.15. We cannot improve in general the estimations on $E$ and $D$. For instance, we cannot have $E(w, z)-w=O\left(|w|^{1 / 2}\right)$. Indeed, we know that $E \circ \phi^{[n]}(1,0)=n^{2} a^{2}+O(n)$ via the functional equation whereas

$\phi_{1}^{[n]}(1,0)+O\left(\left|\phi_{1}^{[n]}(1,0)\right|^{1 / 2}\right)=n^{2} a^{2}+2\left(a^{\prime}+b^{\prime}+c^{\prime}+d^{\prime}\right) n \log (n+1)+O(n)$.

Remark 3.16. It should be observed that the model map $\tau$ is not unique. Indeed, if $(E, D)$ satisfies $(E, D) \circ \phi=(E+2 \alpha D+\beta, D+\beta)$ and if we set $E_{1}=E+D$, then $E_{1} \circ \phi=E_{1}+2 \alpha D+\beta+\alpha$. On the other hand, if we fix the model map $\tau(w, z)=(w+2 \alpha z+\beta, z+\beta)$ and if $(E, D)$ is a solution to $(E, D) \circ \phi=\tau \circ(E, D)$, then any couple $\left(E_{1}, D_{1}\right)$ with $E_{1}=E+\theta_{1} D+\theta_{2}$, $D_{1}=D+\theta_{1}$ remains a solution. Does there exist another kind of solutions? The analog problem in the one-dimensional case (the uniqueness in Abel's equation) was solved quite recently (see [11]).

\section{The hyperbolic case}

\subsection{Presentation of the results}

Throughout this section, we suppose that $\varphi \in \mathcal{D}^{2}\left(e_{1}\right)$ and that $\lambda_{1}<1$. This is the hyperbolic case and the application $\phi$ may be written

$$
\phi(w, z)=\left(\frac{\lambda_{\infty} w}{1-\gamma_{\infty} \frac{z^{2}}{w+1}}+o(|w+1|), \frac{\mu_{\infty} z}{1-\gamma_{\infty} \frac{z^{2}}{w+1}}+O(1)\right) .
$$

This case has already been considered by Bracci and Gentili in [9] when $\gamma_{\infty}=0$ (or equivalently when $\gamma_{1}=0$, namely when there is no pure term in $z^{2}$ in the expansion of $\varphi_{1}$, see also [9, Remark 1.6]). Their result with $\varepsilon=1 / 2$ reads:

Bracci-Gentili's Theorem. Let $\varphi \in \mathcal{D}^{3}\left(e_{1}\right)$ with $\gamma_{\infty}=0, \lambda_{\infty}>1$ and $\left|\mu_{\infty}\right|>\lambda_{\infty}^{1 / 4}$. Then there exists a holomorphic map $\sigma=(E, D): \mathbb{H}_{2} \rightarrow \mathbb{C}^{2}$ such that

$$
\sigma \circ \phi(w, z)=\left(\lambda_{\infty} E(w, z), \mu_{\infty} D(w, z)\right)
$$

Moreover,

$$
E(w, z)-w=O\left(|w|^{1 / 2}\right) \text { and } D(w, z)-z=O\left(|w|^{1 / 4}\right) .
$$

$\sigma$ is the unique map with the previous properties. In the case where $\left|\mu_{\infty}\right| \leq$ $\lambda_{\infty}^{1 / 4}$, the conclusion remains true for the first coordinate. 
Our job in this section consists in building a linear fractional model without any assumption on $\gamma_{\infty}$ and $\mu_{\infty}$. We remark that using (2.3) the condition $\left|\mu_{\infty}\right|^{2}=\lambda_{\infty}$ implies that $\gamma_{\infty}=0$ and $\left|\mu_{\infty}\right|>\lambda_{\infty}^{1 / 4}$, a case that is already covered by Bracci-Gentili's theorem. Thus we may suppose that $\left|\mu_{\infty}\right|<\lambda_{\infty}^{2}$.

Theorem 4.1. Let $\varphi \in \mathcal{D}^{2}\left(e_{1}\right)$ with $\lambda_{\infty}>1,0<\left|\mu_{\infty}\right|<\lambda_{\infty}^{2}$ and $\alpha_{\infty} \neq 0$ if $\mu_{\infty}=1$. Let $m=4$ if $\left|\mu_{\infty}\right| \lambda_{\infty}>1$ and the least integer such that $\left|\mu_{\infty}\right| \lambda_{\infty}^{m / 2-1}>1$ otherwise. We assume that $\varphi \in \mathcal{D}^{m}\left(e_{1}\right)$ and we suppose also that $\lambda_{\infty}^{k} \mu_{\infty} \neq 1$ for any integer $k$. Then there exists a holomorphic map $\sigma=(E, D): \mathbb{H}_{2} \rightarrow \mathbb{C}^{2}$ such that

$$
\begin{aligned}
& \sigma \circ \phi(w, z)=\left(\lambda_{\infty} E(w, z), \mu_{\infty} D(w, z)\right) \quad \text { if } \mu_{\infty} \neq 1 \\
& \sigma \circ \phi(w, z)=\left(\lambda_{\infty} E(w, z), D(w, z)+\alpha_{\infty}\right) \quad \text { if } \mu_{\infty}=1 .
\end{aligned}
$$

Moreover, there is a domain $\Omega$ such that, for any $(w, z) \in \mathbb{H}_{2}, \phi^{[n]}(w, z) \in \Omega$ for $n$ large enough and such that

$$
\begin{gathered}
E(w, z)-w=o(|w|) \text { when }(w, z) \rightarrow \infty \text { along } \Omega, \\
|D(w, z)-z| \leq \frac{P(|z|)}{|w|} \text { for }(w, z) \in \Omega
\end{gathered}
$$

where $P$ is a polynomial. Moreover, $E$ is the unique map with the previous properties and it exists under the weaker assumption $\varphi \in \mathcal{D}^{3}\left(e_{1}\right)$.

The proof of this theorem will be rather long. It begins with the construction of domains such that one of them will be $\Omega$ and with a first analysis on the behavior of $z^{2}(n) / w(n)$ on this domain. We then linearize the first coordinate and we construct $E$. The study of the second one is easier when $\left|\mu_{\infty}\right| \lambda_{\infty}>1$ and we first exhibit $D$ in this case (the value $\mu_{\infty}=1$ requires particular attention). If $\left|\mu_{\infty}\right| \lambda_{\infty} \leq 1$, we have to take into account highorder terms in the expansion of $\phi_{2}$. That is the reason why the regularity of $\varphi$ must increase when $\left|\mu_{\infty}\right|$ decreases. Finally, "resonances" will appear when $\left|\mu_{\infty}\right| \lambda_{\infty}^{k}=1$ for an integer $k$. We will observe that in this case we cannot expect to obtain a linear fractional model under reasonable conditions on the behavior of $D$ near infinity. In such a case, we will provide another type of model map.

\subsection{Three lemmas on the behavior near infinity}

We retain the notations of Section 3, especially for $(w(n), z(n))$ and $\lesssim_{K}$. As for the parabolic non-diagonalizable case, our first job is to find domains $L_{M}$ stable under $\phi$ and to prove that the sequences $(w(n), z(n))$ lie eventually in these domains, uniformly on compact subsets of $\mathbb{H}_{2}$. 
Thus, for $M>0$, we set

$$
L_{M}:=\left\{(w, z) \in \mathbb{H}_{2}:|w|>M \text { and } \frac{|z|}{|w+1|^{1 / 2}}<\frac{1}{\log |w+1|}\right\} .
$$

Lemma 4.2. Let $\varphi \in \mathcal{D}^{3}\left(e_{1}\right)$ with $\lambda_{\infty}>1,\left|\mu_{\infty}\right|^{2}<\lambda_{\infty}$, let $\kappa>\max \left(\frac{1}{\lambda_{\infty}^{1 / 2}}, \frac{\left|\mu_{\infty}\right|}{\lambda_{\infty}^{1 / 2}}\right)$. We may find $M>0$ such that

1. $L_{M}$ is stable under $\phi$.

2. For any $(w, z)$ in $L_{M}$,

$$
\frac{|z(n)|}{|w(n)+1|^{1 / 2}} \lesssim_{\kappa} \kappa^{n} \frac{|z|+1}{|w+1|^{1 / 2}}
$$

Proof. Let $1<\lambda^{\prime}<\lambda_{\infty}<\lambda^{\prime \prime}$ and $\mu^{\prime}>\left|\mu_{\infty}\right|$. For $M$ large enough, the expansion of $\phi$ near infinity gives for any $(w, z)$ in $L_{M}$ :

$$
\begin{gathered}
\lambda^{\prime}|w+1| \leq\left|\phi_{1}(w, z)+1\right| \leq \lambda^{\prime \prime}|w+1| \\
\left|\phi_{2}(w, z)\right| \leq \mu^{\prime}|z|+A
\end{gathered}
$$

where $A \in \mathbb{R}^{+}$. This implies:

$$
\begin{aligned}
\frac{\left|\phi_{2}(w, z)\right|}{\left|\phi_{1}(w, z)+1\right|^{1 / 2}} & \leq \frac{\mu^{\prime}}{\lambda^{\prime 1 / 2}} \frac{|z|}{|w+1|^{1 / 2}}+\frac{A}{\lambda^{\prime 1 / 2}|w+1|^{1 / 2}} \\
& \leq \frac{\mu^{\prime}}{\lambda^{\prime 1 / 2}} \frac{1}{\log \left|\phi_{1}(w, z)+1\right|-\log \left(\lambda^{\prime \prime}\right)}+\frac{A}{\lambda^{\prime 1 / 2}|w+1|^{1 / 2}} .
\end{aligned}
$$

If we choose $\mu^{\prime}$ and $\lambda^{\prime}$ with $\kappa_{0}=\mu^{\prime} / \lambda^{\prime 1 / 2}<1$ and if we choose $M$ large enough we obtain

$$
\frac{\left|\phi_{2}(w, z)\right|}{\left|\phi_{1}(w, z)+1\right|^{1 / 2}} \leq \frac{1}{\log \left|\phi_{1}(w, z)+1\right|}
$$

which means that $L_{M}$ is stable under $\phi$. Finally, an easy induction shows that, provided $(w, z) \in L_{M}$ :

$$
\begin{aligned}
\frac{|z(n)|}{|w(n)+1|^{1 / 2}} & \leq \kappa_{0}^{n}\left(\frac{|z|}{|w+1|^{1 / 2}}+\frac{A}{\lambda^{1 / 2}} \sum_{j=0}^{n-1} \kappa_{0}^{-j-1}|w(j)+1|^{-1 / 2}\right) \\
& \lesssim \kappa_{0}^{n}\left(\frac{|z|}{|w+1|^{1 / 2}}+\sum_{j=0}^{n-1} \frac{1}{\mu^{\prime j}|w+1|^{1 / 2}}\right) \\
& \lesssim \kappa_{0}^{n} \frac{|z|}{|w+1|^{1 / 2}}+\frac{\kappa_{0}^{n}-\kappa_{0}^{n} / \mu^{\prime n}}{1-1 / \mu^{\prime}} \frac{1}{|w+1|^{1 / 2}} .
\end{aligned}
$$

It suffices now to arrange $\lambda^{\prime}$ and $\mu^{\prime}$ so that $\kappa_{0}$ and $\kappa_{0} / \mu^{\prime}$ are less than $\kappa$. 
We point out the interest of working with $L_{M}$ : the term $\frac{1}{1-\gamma_{\infty} \frac{z^{2}}{w+1}}$ does not play any important role in the estimations when $(w, z)$ belongs to $L_{M}$ as this appears clearly in the previous argument. Our aim is now to prove that for a given $(w, z) \in \mathbb{H}_{2},(w(n), z(n))$ lies eventually in $L_{M}, M>0$. This control of $\left|z^{2}(n)\right| /|w(n)+1|$ will be done in two steps.

Lemma 4.3. Let $\varphi \in \mathcal{D}^{2}\left(e_{1}\right)$ with $\lambda_{\infty}>1$ and $\left|\mu_{\infty}\right|^{2}<\lambda_{\infty}$. For any $(w, z)$ in $\mathbb{H}_{2}$, there exists $\omega \in(0,1)$ such that $\frac{\left|z^{2}(n)\right|}{|w(n)+1|} \leq \omega$.

Proof. This follows from [10, Section 3]. Indeed, since $\varphi$ is a self-map of $\mathbb{B}_{2}$ which is of hyperbolic type, the orbit $\varphi^{[n]}(\tilde{w}, \tilde{z})$ stays in a Korányi approach region at $e_{1}$ for every $(\tilde{w}, \tilde{z}) \in \mathbb{B}_{2}$, namely

$$
\frac{\left|1-\varphi_{1}^{[n]}(\tilde{w}, \tilde{z})\right|}{1-\left\|\varphi^{[n]}(\tilde{w}, \tilde{z})\right\|^{2}} \leq M
$$

for some constant $M<+\infty$. Translating this information into the language of the Siegel half-plane, this gives

$$
\frac{|w(n)+1|}{\Re(w(n))-|z(n)|^{2}} \leq M^{\prime}
$$

which can be rewritten

$$
\frac{|z(n)|^{2}}{|w(n)+1|} \leq \frac{\Re(w(n))}{|w(n)+1|}-\frac{1}{M^{\prime}} \leq 1-\frac{1}{M^{\prime}}
$$

Lemma 4.4. Let $\varphi \in \mathcal{D}^{3}\left(e_{1}\right)$ with $\lambda_{\infty}>1$ and $\left|\mu_{\infty}\right|^{2}<\lambda_{\infty}$. Let $K$ be a compact subset of $\mathbb{H}_{2}$ and let $M>0$. There exists an integer $n_{0}$ such that, for every $n \geq n_{0}$, for any $(w, z) \in K,(w(n), z(n))$ belongs to $L_{M}$.

Proof. By compactness of $K$, it suffices to prove the existence of $n_{0}$ for a single $(w, z)$. Let $\omega \in(0,1)$ be given by Lemma 4.3 and let $\kappa=\frac{\left|\mu_{\infty}\right|^{2}}{\lambda_{\infty}\left|1-\gamma_{\infty} \omega\right|}$. We observe that $\kappa<1$ : this follows from the assumption $\left|\mu_{\infty}\right|^{2}<\lambda_{\infty}$ if $\gamma_{\infty}=0$ and from (2.3) otherwise. On the other hand, writing the expansion of $\phi$ near infinity as

$$
\begin{aligned}
\phi_{1}(w, z)+1 & =\frac{\lambda_{\infty}(w+1)}{1-\gamma_{\infty} \frac{z^{2}}{w+1}}\left(1+O\left(|w+1|^{-1 / 2}\right)\right), \\
\phi_{2}(w, z) & =\frac{\mu_{\infty}}{1-\gamma_{\infty} \frac{z^{2}}{w+1}}(z+O(1))
\end{aligned}
$$


we get

$$
\frac{\phi_{2}(w, z)^{2}}{\phi_{1}(w, z)+1}=\frac{\mu_{\infty}^{2}}{\lambda_{\infty}\left(1-\gamma_{\infty} \frac{z^{2}}{w+1}\right)} \frac{z^{2}}{w+1}+O\left(|w+1|^{-1 / 2}\right) .
$$

In particular, one has

$$
\frac{|z(n+1)|^{2}}{|w(n+1)+1|} \leq \kappa \frac{|z(n)|^{2}}{|w(n)+1|}+\frac{A}{|w(n)+1|^{1 / 2}}
$$

where $A>0$. To conclude, we apply Wolff's lemma which shows in particular that $|w(n)+1| \geq \Re(w(n))+1 \gtrsim_{(w, z)} \lambda_{\infty}^{n}$. Coming back to (4.2) an induction shows:

$$
\begin{aligned}
\frac{|z(n)|^{2}}{|w(n)+1|} & \lesssim(w, z) \quad \kappa^{n}\left(1+\sum_{j=0}^{n-1} \frac{1}{\lambda_{\infty}^{j / 2} \kappa^{j}}\right) \\
& \lesssim_{(w, z)} \quad \kappa^{n}+\frac{1}{\lambda_{\infty}^{n / 2}}
\end{aligned}
$$

Finally, equation (4.1) shows that $|w(n)+1| \lesssim\left(\lambda^{\prime}\right)^{n}|w+1|$ for some positive constant $\lambda^{\prime}$. Taking the logarithm, we get the lemma.

\subsection{Linearization of the first coordinate}

The study of the first coordinate is much easier in the hyperbolic case than the study of the second one.

Proposition 4.5. Let $\varphi \in \mathcal{D}^{3}\left(e_{1}\right)$ with $\lambda_{\infty}>1$ and $\left|\mu_{\infty}\right|^{2}<\lambda_{\infty}$. There exists a holomorphic function $E: \mathbb{H}_{2} \rightarrow \mathbb{C}$ such that

$$
E \circ \phi=\lambda_{\infty} E
$$

Moreover, for $M$ large enough, one has $E(w, z)-w=o(|w|)$ when $(w, z) \rightarrow \infty$ along $L_{M}$. E is the unique map with the previous properties.

Proof. In view of Lemma 4.4, it suffices to construct $E$ on the set $L_{M}$ given by Lemma 4.2 . Hence, consider $(w, z) \in L_{M}$ and write

$$
\phi_{1}(w, z)=\frac{\lambda_{\infty} w}{1-\gamma_{\infty} \frac{z^{2}}{w+1}}+\varepsilon_{1}(w, z) \text { where }\left|\varepsilon_{1}(w, z)\right| \lesssim|w+1|^{1 / 2} .
$$

By induction, we get

$$
w(n)=\lambda_{\infty}^{n}\left(\left(\prod_{j=0}^{n-1} \frac{1}{1-\gamma_{\infty} \frac{z^{2}(j)}{w(j)+1}}\right) w+\sum_{j=0}^{n-1}\left(\prod_{k=j+1}^{n-1} \frac{1}{1-\gamma_{\infty} \frac{z^{2}(k)}{w(k)+1}}\right) \frac{\varepsilon_{1}(w(j), z(j))}{\lambda_{\infty}^{j+1}}\right) .
$$


Now, an application of Lemma 4.2 with $\kappa<1$ gives

$$
\sum_{j \geq 0} \frac{|z(j)|^{2}}{|w(j)+1|} \lesssim \frac{|z|^{2}+1}{|w+1|} \lesssim 1 .
$$

This gives successively, for any $n \geq 1$ and any $0 \leq j \leq n-1$

$$
\prod_{k=j+1}^{n-1}\left|\frac{1}{1-\gamma_{\infty} \frac{z^{2}(j)}{w(j)+1}}\right| \lesssim 1
$$

and

$$
\begin{aligned}
\left|\prod_{k=j+1}^{n-1} \frac{1}{1-\gamma_{\infty} \frac{z^{2}(j)}{w(j)+1}}-1\right| & \leq \sum_{k=j+1}^{n-1}\left(\prod_{l=k+1}^{n-1}\left|\frac{1}{1-\gamma_{\infty} \frac{z^{2}(l)}{w(l)+1}}\right|\right)\left|\frac{1}{1-\gamma_{\infty} \frac{z^{2}(k)}{w(k)+1}}-1\right| \\
& \lesssim \sum_{k=j+1}^{n-1} \frac{\left|z^{2}(k)\right|}{|w(k)+1|} \\
& \lesssim \frac{|z|^{2}+1}{|w+1|} .
\end{aligned}
$$

Moreover, using the notations of the proof of Lemma 4.2, one has

$$
\left|\varepsilon_{1}(w(j), z(j))\right| \lesssim|w(j)+1|^{1 / 2} \leq\left(\lambda^{\prime \prime}\right)^{j / 2}|w+1|^{1 / 2} .
$$

If we choose $\left(\lambda^{\prime \prime}\right)^{1 / 2}<\lambda_{\infty}$ and if we adapt the choice of $M$ accordingly, we finally get that $\frac{|w(n)|}{\lambda_{\infty}^{n}}$ converges uniformly on $L_{M}$ to a holomorphic function $E$ satisfying

$$
\begin{aligned}
|E(w, z)-w| & \lesssim \limsup _{n}\left|\prod_{j=0}^{n-1} \frac{1}{1-\gamma_{\infty} \frac{z^{2}(j)}{w(j)+1}}-1\right||w|+\sum_{j=0}^{+\infty}\left(\frac{\left(\lambda^{\prime \prime}\right)^{1 / 2}}{\lambda_{\infty}}\right)^{j}|w+1|^{1 / 2} \\
& \lesssim|z|^{2}+|w+1|^{1 / 2} .
\end{aligned}
$$

This gives the estimation, since when $(w, z) \rightarrow \infty$ along $L_{M}$ one has $|z|^{2}=$ $o(|w|)$. E satisfies the functional equation $E \circ \phi=\lambda_{\infty} E$ since

$$
E \circ \phi(w, z)=\lim _{n \rightarrow+\infty} \frac{w(n+1)}{\lambda_{\infty}^{n}}=\lambda_{\infty} \lim _{n \rightarrow+\infty} \frac{w(n+1)}{\lambda_{\infty}^{n+1}}=\lambda_{\infty} E(w, z) .
$$

Let us conclude by showing the uniqueness of $E$. Suppose that $E^{\prime}$ is another solution with the same asymptotic control. Fix $(w, z)$ in $\mathbb{H}_{2}$ and observe that

$$
\left|\left(E-E^{\prime}\right)(w(n), z(n))\right|=\left|\lambda_{\infty}\right|^{n}\left|E(w, z)-E^{\prime}(w, z)\right|
$$

whereas

$$
\left|\left(E-E^{\prime}\right)(w(n), z(n))\right|=o(w(n))=o\left(\lambda_{\infty}^{n}\right) .
$$

This implies that $E(w, z)=E^{\prime}(w, z)$. 
4.4. Linearization of the second coordinate, case $\left|\mu_{\infty}\right| \lambda_{\infty}>1, \mu_{\infty} \neq 1$ In this subsection, we complete the proof of Theorem 4.1 when $\left|\mu_{\infty}\right| \lambda_{\infty}>1$ and $\mu_{\infty} \neq 1$ (or $\mu_{1} \neq \lambda_{1}$ ). As explained in Section 2, up to conjugation by a Heisenberg translation (which does not change the statement of Theorem 4.1) we may suppose that $\alpha_{1} \neq 0$. Since $\varphi$ belongs to $\mathcal{D}^{4}\left(e_{1}\right)$, collecting the terms of order 0 (respectively $-1 / 2$ ) together, we may write $\phi_{2}$ as

$$
\phi_{2}(w, z)=\frac{\mu_{\infty} z}{1-\gamma_{\infty} \frac{z^{2}}{w+1}}+\frac{z^{2}}{w+1} A(w, z)+\frac{z}{w+1} B(w, z)+\varepsilon(w, z)
$$

where $|\varepsilon(w, z)| \lesssim|w+1|^{-1}$ and the functions $A, B$ are uniformly bounded on $\mathbb{H}_{2}$. It suffices to construct $D$ on a suitably chosen $L_{M}$ and an iteration shows that

$$
\begin{aligned}
z(n) & =\mu_{\infty}^{n}\left(\prod_{j=0}^{n-1} \frac{1}{1-\gamma_{\infty} \frac{z^{2}(j)}{w(j)+1}} z+\sum_{j=0}^{n-1}\left(\prod_{k=j+1}^{n-1} \frac{1}{1-\gamma_{\infty} \frac{z^{2}(k)}{w(k)+1}}\right) \frac{1}{\mu_{\infty}^{j+1}} \times\right. \\
& \left.\times\left(\frac{z^{2}(j)}{w(j)+1} A(w(j), z(j))+\frac{z(j)}{w(j)+1} B(w(j), z(j))+\varepsilon(w(j), z(j))\right)\right) .
\end{aligned}
$$

In the proof of Proposition 4.5, we have shown that the product

$$
\prod_{k=j+1}^{n-1}\left|\frac{1}{1-\gamma_{\infty} \frac{z^{2}(k)}{w(k)+1}}\right|
$$

is uniformly bounded on some $L_{M}$. Thus we find

$$
\sum_{j=0}^{n-1}\left|\prod_{k=j+1}^{n-1} \frac{1}{1-\gamma_{\infty} \frac{z^{2}(k)}{w(k)+1}}\right|\left|\frac{1}{\mu_{\infty}^{j+1}}\right|\left|\frac{z^{2}(j)}{w(j)+1}\right||A(w(j), z(j))| \sum_{\kappa} \sum_{j=0}^{+\infty} \frac{\kappa^{2 j}}{\mid \mu_{\infty}^{j}} \frac{|z|^{2}+1}{|w+1|}
$$

where $\kappa$ is any number greater than $\max \left(\frac{1}{\lambda_{\infty}^{1 / 2}}, \frac{\left|\mu_{\infty}\right|}{\lambda_{\infty}^{1 / 2}}\right)$. Because $\frac{1}{\left|\mu_{\infty}\right| \lambda_{\infty}}<1$ and $\frac{\left|\mu_{\infty}\right|}{\lambda_{\infty}}<1$ we may adjust $\kappa$ so that the previous series converges. For the second term, we have:

$$
\begin{aligned}
\sum_{j=0}^{n-1}\left|\prod_{k=j+1}^{n-1} \frac{1}{1-\gamma_{\infty} \frac{z^{2}(k)}{w(k)+1}}\right| & \left|\frac{z(j) /\left|\mu_{\infty}\right|^{j}}{w(j)+1}\right||B(w(j), z(j))| \\
& \lesssim_{\kappa} \sum_{j=0}^{+\infty} \frac{\kappa^{j}}{\left|\mu_{\infty}\right|^{j}} \frac{1}{|w(j)+1|^{\frac{1}{2}}} \frac{|z|}{|w+1|} \\
& \lesssim_{\kappa} \sum_{j=0}^{+\infty}\left(\frac{\kappa}{\left|\mu_{\infty}\right|\left(\lambda^{\prime}\right)^{1 / 2}}\right)^{j} \frac{|z|+1}{|w+1|}
\end{aligned}
$$


where $\lambda^{\prime}$ is any constant smaller than $\lambda_{\infty}$. Since $\frac{1}{\left|\mu_{\infty}\right| \lambda_{\infty}}<1$ and $\frac{1}{\lambda_{\infty}}<1$, we may adjust $\kappa$ and $\lambda^{\prime}$ so that the previous series converges. For the third term, we have

$$
\sum_{j=0}^{n-1}\left|\prod_{k=j+1}^{n-1} \frac{1}{1-\gamma_{\infty} \frac{z^{2}(k)}{w(k)+1}}\right|\left|\frac{1}{\mu_{\infty}^{j}}\right||\varepsilon(w(j), z(j))| \lesssim \sum_{j=0}^{+\infty}\left|\frac{1}{\mu_{\infty} \lambda^{\prime}}\right|^{j} \frac{1}{|w+1|}
$$

and this series is convergent provided $\lambda^{\prime}$ is close enough to $\lambda_{\infty}$. Using the uniform convergence of

$$
\prod_{j=0}^{n} \frac{1}{1-\gamma_{\infty} \frac{z^{2}(j)}{w(j)+1}}
$$

on $L_{M}$, we get that $\frac{z(n)}{\mu_{\infty}^{n}}$ converges uniformly on $L_{M}$ to a holomorphic function $D$ which satisfies, as for the first coordinate, $D \circ \phi=\mu_{\infty} D$. On $L_{M}, D$ satisfies

$$
\begin{aligned}
|D(w, z)-z| & \lesssim \limsup _{n}\left|\prod_{j=1}^{n-1} \frac{1}{1-\gamma_{\infty} \frac{z^{2}(j)}{w(j)+1}}-1\right||z|+\frac{|z|^{2}+1}{|w+1|} \\
& \lesssim(|z|+1) \frac{|z|^{2}+1}{|w+1|}
\end{aligned}
$$

This shows that $D$ is not constant.

Remark 4.6. We can prove that $D$ is the unique solution which satisfies the relation (4.4). Indeed, if we replace $(w, z)$ by $(w(n), z(n))$ in this inequality and using our previous estimates on the behavior of $w(n)$ and $z(n)$ this gives

$$
\begin{aligned}
|D(w(n), z(n))-z(n)| & \lesssim_{(w, z)} \quad\left(\left|\mu_{\infty}\right|^{n}+1\right) \frac{\left|\mu_{\infty}\right|^{2 n}+1}{\lambda_{\infty}^{n}} \\
& \lesssim_{(w, z)} \frac{\left|\mu_{\infty}\right|^{3 n}+1}{\lambda_{\infty}^{n}} .
\end{aligned}
$$

On the other hand, for $D(w, z) \neq 0, z(n) \sim \mu_{\infty}^{n} D(w, z)$. Since $\left|\mu_{\infty}\right|^{2}<\lambda_{\infty}$ and $\left|\mu_{\infty} \lambda_{\infty}\right|>1$, we obtain that $D(w(n), z(n))=z(n)+o(z(n))$. The uniqueness of $D$ is proved along the same lines than the uniqueness of $E$. Indeed, let $D, D^{\prime}$ be two solutions and let $\Omega_{0}$ be the open non-empty set $\left\{(w, z) \in \mathbb{H}_{2}: D(w, z) \neq 0\right.$ and $\left.D^{\prime}(w, z) \neq 0\right\}$. The asymptotic behavior of $D$ and $D^{\prime}$ ensures that for $(w, z) \in \Omega_{0}$ one has

$$
\left|\left(D-D^{\prime}\right)(w(n), z(n))\right|=o(z(n))=o\left(\mu_{\infty}^{n}\right)
$$

whereas $\left(D-D^{\prime}\right)(w(n), z(n))=\mu_{\infty}^{n}\left(D-D^{\prime}\right)(w, z)$. Thus $D$ and $D^{\prime}$ coincide on $\Omega_{0}$ hence on $\mathbb{H}_{2}$. 


\subsection{Linearization of the second coordinate, case $\mu_{\infty}=1$}

We finish here the proof of Theorem 4.1 in the case where $\mu_{\infty}=1$ and $\alpha_{\infty} \neq 0$. We write

$$
\phi_{2}(w, z)=\frac{z-\alpha_{\infty}}{1-\gamma_{\infty} \frac{z^{2}}{w+1}}+\frac{z^{2}}{w+1} A(w, z)+\frac{z}{w+1} B(w, z)+\varepsilon(w, z)
$$

where $|\varepsilon(w, z)| \lesssim|w+1|^{-1}$ and $A, B$ are bounded functions on $\mathbb{H}_{2}$. Iterating the function, we have now

$$
\begin{aligned}
& z(n)=\prod_{j=0}^{n-1} \frac{1}{1-\gamma_{\infty} \frac{z^{2}(j)}{w(j)+1}} z+\sum_{j=0}^{n-1}\left(\prod_{k=j+1}^{n-1} \frac{1}{1-\gamma_{\infty} \frac{z^{2}(k)}{w(k)+1}}\right) \times \\
& \times\left(-\alpha_{\infty}+\frac{z^{2}(j)}{w(j)+1} A(w(j), z(j))+\frac{z(j)}{w(j)+1} B(w(j), z(j))+\varepsilon(w(j), z(j))\right) .
\end{aligned}
$$

On a suitable domain $L_{M}$, the product

$$
\prod_{k=j+1}^{n-1} \frac{1}{1-\gamma_{\infty} \frac{z^{2}(k)}{w(k)+1}}
$$

is uniformly bounded. This yields

$$
\left|\sum_{j=0}^{n-1}\left(\prod_{k=j+1}^{n-1} \frac{1}{1-\gamma_{\infty} \frac{z^{2}(k)}{w(k)+1}}\right) \alpha_{\infty}\right| \lesssim \sum_{j=0}^{n-1} 1=n .
$$

The other terms are estimated exactly like in the previous subsection, replacing everywhere $\mu_{\infty}$ by 1 . Thus, one has $|z(n)| \lesssim|z|+n$.

Next we decompose $z(n)$ into $z(n)=-n \alpha_{\infty}+z^{\prime}(n)$. Using Lemma 4.2, we have

$$
\begin{aligned}
& \left|z^{\prime}(n+1)-z^{\prime}(n)\right| \leq\left|\frac{1}{1-\gamma_{\infty} \frac{z^{2}(n)}{w(n)+1}}-1\right|\left|z(n)-\alpha_{\infty}\right| \\
& \quad+\left|\frac{z^{2}(n)}{w(n)+1} A(w(n), z(n))\right|+\mid \frac{z(n)}{w(n)+1} B(w(n), z(n)|+| \varepsilon(w(n), z(n)) \mid \\
& \lesssim_{\kappa} \kappa^{2 n} \frac{|z|^{2}+1}{|w+1|}(|z|+n)+\kappa^{2 n} \frac{|z|^{2}+1}{|w+1|}+\frac{\kappa^{n}}{\lambda_{\infty}^{n}} \frac{|z|+1}{|w+1|}+\frac{1}{\lambda_{\infty}^{n}|w|} .
\end{aligned}
$$

In particular, we get that $z^{\prime}(n)$ converges uniformly on $L_{M}$ to a holomorphic function $D$ satisfying (as for the Heisenberg translation case) the functional equation $D \circ \phi=D-\alpha_{\infty}$. A summation ensures that

$$
|D(w, z)-z| \leq \sum_{n \geq 0}\left|z^{\prime}(n+1)-z^{\prime}(n)\right| \leq \frac{P(|z|)}{|w|}
$$

where $P$ is a polynomial of degree 3 . Moreover its uniqueness with the previous asymptotic behavior is proved along the same lines as above. 


\subsection{Linearization of the second coordinate, case $\left|\mu_{\infty}\right| \lambda_{\infty} \leq 1$.}

Our method of proof breaks down while $\left|\mu_{\infty}\right| \lambda_{\infty} \leq 1$. This is not just a technical problem. The following very simple example points out that the behavior of $z(n)$ is not given by $\mu_{\infty}^{n} z$.

Example 4.7. Let $\phi(w, z)=(2 w+2, z / 4+1 /(w+1))$ which is a holomorphic self-map of $\mathbb{H}_{2}$. It is easy to prove that for a given $(w, z) \in \mathbb{H}_{2}$, one has

$$
\begin{aligned}
& w(n)=2^{n}(w+2)-2 \sim 2^{n}(w+2) \\
& z(n)=\frac{1}{4^{n}}\left(z+\sum_{j=0}^{n-1} \frac{4^{j}}{2^{j}(w+2)-2}\right) \sim \frac{1}{2^{n-1}(w+2)} .
\end{aligned}
$$

If we compare this example with the table of orbits for linear fractional maps given in Section 2.4 then we can give a (negative) answer to a question raised by Bracci and Poggi-Corradini in [10]:

How closely are the orbits of $\phi$ trying to imitate the behavior of a corresponding linear fractional map of $\mathbb{H}_{2}$ ?

For $\phi$ given by the previous formula, the imitation is very bad!

Thus a new difficulty arises in several variables. However, even for this kind of maps, we will be able to provide a linear fractional model. The novelty is: when $\left|\mu_{\infty}\right|$ becomes smaller and smaller, other terms $z^{p}(n) / w^{q}(n)$ come into play. This explains why we need more regularity when $\left|\mu_{\infty}\right|$ decreases.

So let us suppose that $\left|\mu_{\infty}\right| \lambda_{\infty} \leq 1$ and that $\mu_{\infty} \lambda_{\infty}^{k} \neq 1$ for any $k \geq 1$. We consider $m \geq 4$ the least integer such that $\left|\mu_{\infty}\right| \lambda_{\infty}^{m / 2-1} \geq 1$. Let us first produce an enhancement of Lemma 4.2 with these new assumptions.

Lemma 4.8. Let $\varphi$ satisfying the previous assumptions and let $\kappa>\frac{1}{\lambda_{\infty}^{3 / 2}}$. There exists $M>0$ such that, for any $(w, z) \in L_{M}$, one has

$$
\frac{|z(n)|}{|w(n)+1|^{1 / 2}} \lesssim_{\kappa} \kappa^{n}\left(\frac{|z|}{|w+1|^{1 / 2}}+\frac{|z|^{2}+1}{|w+1|^{3 / 2}}\right) .
$$

Proof. Let $\lambda^{\prime}<\lambda_{\infty}$. We write and majorize $|z(n)|$ as in Section 4.4, using Lemma 4.2 with $\kappa=1 /\left(\lambda^{\prime}\right)^{1 / 2}$ (observe that $\max \left(1 / \lambda_{\infty}^{1 / 2},\left|\mu_{\infty}\right| / \lambda_{\infty}^{1 / 2}\right)=$ $\left.1 / \lambda_{\infty}^{1 / 2}\right)$ :

$$
|z(n)| \lesssim\left|\mu_{\infty}\right|^{n}\left(|z|+\sum_{j=0}^{n-1} \frac{|z|^{2}+1}{\left(\lambda^{\prime}\right)^{j}\left|\mu_{\infty}\right|^{j}|w+1|}\right) \lesssim\left|\mu_{\infty}\right|^{n}|z|+\frac{|z|^{2}+1}{\left(\lambda^{\prime}\right)^{n}|w+1|}
$$


Thus,

$$
\frac{|z(n)|}{|w(n)+1|^{1 / 2}} \lesssim \frac{\left|\mu_{\infty}\right|^{n}|z|}{\left(\lambda^{\prime}\right)^{n / 2}|w+1|^{1 / 2}}+\frac{|z|^{2}+1}{\left(\lambda^{\prime}\right)^{3 n / 2}|w+1|^{3 / 2}}
$$

We conclude by noting that $\frac{\left|\mu_{\infty}\right|}{\lambda_{\infty}^{1 / 2}} \leq \frac{1}{\lambda_{\infty}^{3 / 2}}$.

We need yet another way to describe $\phi_{1}$ and $\phi_{2}$ near infinity. As Example 4.7 shows, $w(n)$ behaves like $\lambda_{\infty}^{n}$ and $z(n)$ behaves like $1 / \lambda_{\infty}^{n}$. This leads us to order the terms $\frac{z^{p}}{w^{q}}$ with respect to the value of $p+q$. To this aim, let $1 \leq l \leq m / 2-1$. We keep the notations of Corollary 2.7 and we decompose each term in the following way:

$$
\begin{aligned}
E_{p, q}\left(\frac{z^{2}}{w}\right) \frac{z^{p}}{w^{q-1}} & =\sum_{k \geq 0} a_{k} \frac{z^{p+2 k}}{w^{q-1+k}} \\
& =\sum_{k \leq k_{0}} a_{k} \frac{z^{p+2 k}}{w^{q-1+k}}+\frac{z^{p+2 k_{0}+2}}{w^{q+k_{0}}} E_{p, q}^{\prime}\left(\frac{z^{2}}{w}\right)
\end{aligned}
$$

where $k_{0}$ is the biggest integer such that $p+2 k_{0}+q-1+k_{0} \leq l-1$ and $E_{p, q}^{\prime}$ is bounded on $\overline{\mathbb{D}}$. Let $p^{\prime}=\min \left(p+2 k_{0}+2, l\right)$. We factorize

$$
\frac{z^{p+2 k_{0}+2}}{w^{q+k_{0}}}=\frac{z^{p^{\prime}}}{w^{l-p^{\prime}}} \frac{z^{p+2 k_{0}+2-p^{\prime}}}{w^{q+k_{0}-l+p^{\prime}}} .
$$

Now for any $(p, q) \in \mathcal{E}_{m} \cup\{(2,1)\}$ it is easy to check that $\left(p+2 k_{0}+2-\right.$ $\left.p^{\prime}\right)-2\left(q+k_{0}-l+p^{\prime}\right) \leq 2$ (we distinguish $p^{\prime}=l$ and $\left.p^{\prime}=p+2 k_{0}+2\right)$. This yields

$$
\frac{z^{p+2 k_{0}+2}}{w^{q+k_{0}}}=\frac{z^{p^{\prime}}}{w^{l-p^{\prime}}} E_{p, q}^{\prime \prime}(w, z)
$$

where $\left|E_{p, q}^{\prime \prime}(w, z)\right| \leq P(|z|), \operatorname{deg}(P) \leq 2$. We then put together the terms $\frac{z^{p+2 k}}{w^{q-1+k}}$ with the same value of $p+2 k+q-1+k$. We finally find that on $L_{M}, \phi_{1}$ may be written

$$
\phi_{1}(w, z)=\lambda_{\infty} w+\sum_{j=0}^{l-1} \sum_{p+q=j} a_{p, q} \frac{z^{p}}{w^{q}}+\sum_{p+q=l} \frac{z^{p}}{w^{q}} E_{p, q}(w, z)+\varepsilon_{1}(w, z)
$$

with $a_{p, q} \in \mathbb{C},\left|E_{p, q}(w, z)\right| \leq P(|z|)$ on $L_{M}$, $\operatorname{deg}(P) \leq 2$, and $\left|\varepsilon_{1}(w, z)\right| \lesssim_{M}$ $\frac{1}{|w|^{m / 2-2}}$. We do the same for the second coordinate, except that we retain the terms until order $l$ (instead of order $l-1$ ). With $k_{0}$ the biggest integer such that $u+v+3 k_{0} \leq l$ and $u^{\prime}=\min \left(u+2 k_{0}+2, l+1\right)$, we write

$$
\frac{z^{u+2 k_{0}+2}}{w^{v+k_{0}+1}}=\frac{z^{u^{\prime}}}{w^{l+1-u^{\prime}}} \frac{z^{u+2 k_{0}+2-u^{\prime}}}{w^{v+k_{0}-l+u^{\prime}}}
$$


and we observe that $u+2 k_{0}+2-u^{\prime}-2\left(v+k_{0}-l+u^{\prime}\right) \leq 0$ for $(u, v) \in$ $\mathcal{F}_{m} \cup\{(3,1)\}$. Since a couple $(u, v)$ in $\mathcal{F}_{m}$ satisfies $v \neq 0, \phi_{2}$ may thus be written on $L_{M}$

$$
\phi_{2}(w, z)=\mu_{\infty} z+\sum_{j=1}^{l} \sum_{\substack{u+v=j \\ v>0}} b_{u, v} \frac{z^{u}}{w^{v}}+\sum_{\substack{u+v=l+1 \\ v>0}} \frac{z^{u}}{w^{v}} F_{u, v}(w, z)+\varepsilon_{2}(w, z)
$$

with $b_{u, v} \in \mathbb{C},\left|F_{u, v}(w, z)\right| \lesssim_{M} 1$ on $L_{M}$ and $\left|\varepsilon_{2}(w, z)\right| \lesssim_{M} \frac{1}{|w|^{m / 2-1}}$. In fact, considering more precisely what $\mathcal{E}_{m}$ and $\mathcal{F}_{m}$ are, we could prove that many of the $a_{p, q}$ 's and $b_{u, v}$ 's are zero. However, this will not affect our work. We will often use iterate versions of the previous expansions. Namely, for $M$ large enough and $(w, z) \in L_{M}$, one has

$$
\begin{aligned}
w(n)=\lambda_{\infty}^{n}\left(w+\sum_{k=0}^{n-1} \frac{1}{\lambda_{\infty}^{k+1}}(\right. & \sum_{j=0}^{l-1} \sum_{p+q=j} a_{p, q} \frac{z^{p}(k)}{w^{q}(k)} \\
& \left.\left.+\sum_{p+q=l} \frac{z^{p}(k)}{w^{q}(k)} E_{p, q}(w(k), z(k))+\varepsilon_{1}(w(k), z(k))\right)\right) .
\end{aligned}
$$

Since $E(w, z)=\lim _{n} \frac{w(n)}{\lambda_{\infty}^{n}}$, it comes

$$
\begin{aligned}
w(n)=\lambda_{\infty}^{n} E(w, z)-\lambda_{\infty}^{n} \sum_{k=n}^{+\infty} \frac{1}{\lambda_{\infty}^{k+1}}\left(\sum_{j=0}^{l-1} \sum_{p+q=j} a_{p, q} \frac{z^{p}(k)}{w^{q}(k)}\right. \\
\left.+\sum_{p+q=l} \frac{z^{p}(k)}{w^{q}(k)} E_{p, q}(w(k), z(k))+\varepsilon_{1}(w(k), z(k))\right) .
\end{aligned}
$$

For $z(n)$ we simply write

$$
\begin{aligned}
(4.6) z(n)=\mu_{\infty}^{n}( & z+\sum_{k=0}^{n-1} \frac{1}{\mu_{\infty}^{k+1}}\left(\sum_{j=0}^{l} \sum_{\substack{u+v=j \\
v>0}} b_{u, v} \frac{z^{u}(k)}{w^{v}(k)}+\right. \\
& \left.\left.+\sum_{\substack{u+v=l+1 \\
v>0}} \frac{z^{u}(k)}{w^{v}(k)} F_{u, v}(w(k), z(k))+\varepsilon_{2}(w(k), z(k))\right)\right) .
\end{aligned}
$$

The forthcoming proposition is the main step for this part of the proof. 
Proposition 4.9. Let $l$ be in $[1, m / 2-1]$. For any $(w, z)$ in $L_{M}$ for $M$ large enough, one has

$$
\begin{aligned}
& w(n)=\lambda_{\infty}^{n} E(w, z)+a_{2}+\frac{a_{3}}{\lambda_{\infty}^{n} E(w, z)}+\cdots+\frac{a_{l}}{\lambda_{\infty}^{(l-2) n} E^{l-2}(w, z)}+\delta_{n}(w, z) \\
& z(n)=\frac{b_{1}}{\lambda_{\infty}^{n} E(w, z)}+\cdots+\frac{b_{l}}{\lambda_{\infty}^{l n} E^{l}(w, z)}+\mu_{\infty}^{n} z+\eta_{n}(w, z)
\end{aligned}
$$

where $a_{i}, b_{j} \in \mathbb{C},\left|\lambda_{\infty}^{(l-1) n} \delta_{n}(w, z)\right| \lesssim_{M} P(|z|)$ and

$$
\left|\eta_{n}(w, z)\right| \lesssim_{M} \frac{Q(|z|)}{|w|} \max \left(1 / \lambda_{\infty}^{(l+1) n},\left|\mu_{\infty}\right|^{n}\right),
$$

on $L_{M}, P, Q$ being polynomials.

Proof. We prove this by induction. We begin by applying (4.5) with $l=0$ to get

$$
\begin{aligned}
\left|w(n)-\lambda_{\infty}^{n} E(w, z)\right| & \leq \lambda_{\infty}^{n} \sum_{k=n}^{+\infty} \frac{1}{\lambda_{\infty}^{k+1}}\left(\left|E_{0,0}(w(k), z(k))\right|+\left|\varepsilon_{1}(w(k), z(k))\right|\right) \\
& \lesssim_{M} \quad \lambda_{\infty}^{n} \sum_{k=n}^{+\infty} \frac{|z(k)|^{2}+1}{\lambda_{\infty}^{k}} \lesssim_{M}|z|^{2}+1
\end{aligned}
$$

since Lemma 4.8 shows that $|z(k)|$ is dominated by $|z|+1$. This inequality yields in particular that on a well-chosen $L_{M}$ one has $|w(n)| \gtrsim_{M} \lambda_{\infty}^{n}|w|$. Concerning $z(n)$ we use (4.6) with $l=1$ to write

$$
\begin{aligned}
z(n)=\mu_{\infty}^{n} z+\mu_{\infty}^{n} \sum_{k=0}^{n-1} \frac{1}{\mu_{\infty}^{k+1}}\left(\frac{b_{0,1}}{w(k)}+\frac{1}{w^{2}(k)} F_{2,0}(w(k), z(k))\right. \\
\left.\quad+\frac{z(k)}{w(k)} F_{1,1}(w(k), z(k))+\varepsilon_{2}(w(k), z(k))\right) \\
=\mu_{\infty}^{n} z+\mu_{\infty}^{n} \sum_{k=0}^{n-1} \frac{1}{\mu_{\infty}^{k+1}}\left(\frac{b_{0,1}}{\lambda_{\infty}^{k} E(w, z)}+R(w(k), z(k))\right) \\
=\mu_{\infty}^{n} z+\mu_{\infty}^{n-1} b_{0,1}^{\prime}\left(\frac{-1}{\lambda_{\infty}^{n} \mu_{\infty}^{n} E(w, z)}+\frac{1}{E(w, z)}\right)+\mu_{\infty}^{n} \sum_{k=0}^{n-1} \frac{R(w(k), z(k))}{\mu_{\infty}^{k+1}} .
\end{aligned}
$$

We recall that $|E(w, z)| \gtrsim_{M}|w|$ on $L_{M}$ which explains why the term $\frac{\mu_{\infty}^{n}}{E(w, z)}$ goes into $\eta_{n}(w, z)$. It remains to estimate the four terms which come in $R(w(k), z(k))$. Using our work on $w(n)$, one has:

$$
\left|\frac{1}{w(k)}-\frac{1}{\lambda_{\infty}^{k} E(w, z)}\right| \lesssim_{M} \frac{P(|z|)}{\lambda_{\infty}^{2 k}|w|^{2}} .
$$


Thus,

$$
\left|\mu_{\infty}^{n} \sum_{k=0}^{n-1} \frac{1}{\mu_{\infty}^{k+1}}\left(\frac{1}{w(k)}-\frac{1}{\lambda_{\infty}^{k} E(w, z)}\right)\right| \lesssim_{M} \max \left(\frac{1}{\lambda_{\infty}^{2 n}},\left|\mu_{\infty}\right|^{n}\right) \frac{P(|z|)}{|w|^{2}}
$$

(here, the fact that $\mu_{\infty} \lambda_{\infty}^{r} \neq 1$ is crucial). The term with $\frac{1}{w^{2}(k)}$ and that with $\varepsilon_{2}(w(k), z(k))$ are handled under a similar fashion. Indeed, the first one is dominated by $\frac{1}{\lambda_{\infty}^{2 k}|w|^{2}}$ while the second one is dominated by $\frac{1}{\lambda_{\infty}^{(m / 2-1) k}|w|}$ (we observe that $\left.\frac{1}{\lambda_{\infty}^{m / 2-1}} \leq\left|\mu_{\infty}\right|\right)$. To achieve the first step we have to consider $|z(k)| /|w(k)|$. We proceed in two steps. First, fixing $\kappa \in\left(\frac{1}{\lambda_{\infty}^{3 / 2}}, 1\right)$, using Lemma 4.8 and the estimation $\lambda_{\infty}^{n}|w| \lesssim_{M}|w(n)|$, one has

$$
\begin{aligned}
\left|\mu_{\infty}\right|^{n} \sum_{k=0}^{n} \frac{1}{\left|\mu_{\infty}\right|^{k+1}} \frac{|z(k)|}{|w(k)|}\left|F_{1,1}(w(k), z(k))\right| & \lesssim_{M}\left|\mu_{\infty}\right|^{n} \sum_{k=0}^{n} \frac{\kappa^{k}}{\left|\mu_{\infty}\right|^{k} \lambda_{\infty}^{k / 2}} \frac{P(|z|)}{|w|} \\
& \lesssim_{M} \max \left(\left|\mu_{\infty}\right|^{n}, \frac{\kappa^{n}}{\lambda_{\infty}^{n / 2}}\right) \frac{P(|z|)}{|w|} .
\end{aligned}
$$

This does not give immediately the desired estimation since $\kappa / \lambda_{\infty}^{1 / 2}>\lambda_{\infty}^{-2}$. However putting all the previous inequalities together implies that

$$
\begin{aligned}
|z(n)| & \lesssim_{M}\left(\left|\mu_{\infty}\right|^{n}+\frac{1}{\left|\lambda_{\infty}\right|^{n}}+\frac{\kappa^{n}}{\lambda_{\infty}^{n / 2}}\right) P(|z|) \\
& \lesssim_{M} \frac{P(|z|)}{\lambda_{\infty}^{n}} .
\end{aligned}
$$

Second we can come back to (4.7) and we replace in it the estimate on $z(k)$ given by Lemma 4.8 by the previous one. This allows us to replace $\kappa^{n} / \lambda_{\infty}^{n / 2}$ by $1 / \lambda_{\infty}^{2 n}$ and to complete the first step of the induction.

Suppose now that the proof has been done until step $l-1$ and let us show how to derive the result at step $l$. We need first to study how to replace the terms $\frac{z^{p}(k)}{w^{q}(k)}$ by $\frac{1}{\lambda_{\infty}^{p+q} E^{p+q}(w, z)}$.

Lemma 4.10. For any $q \geq 1$, one has

$$
\frac{1}{w^{q}(k)}=\frac{c_{q}}{\lambda_{\infty}^{k q} E^{q}(w, z)}+\cdots+\frac{c_{l-1}}{\lambda_{\infty}^{(l-1) k} E^{l-1}(w, z)}+\varepsilon_{k}(w, z)
$$

where $c_{i} \in \mathbb{C}$,

$$
\left|\lambda_{\infty}^{k l} \varepsilon_{k}(w, z)\right| \lesssim_{M} \frac{P(|z|)}{|w|} .
$$


Proof. It is sufficient to prove the lemma for $q=1$ to deduce it for all values of $q$. Thus, we prove by induction on $r \leq l-1$ that

$$
\frac{1}{w(k)}=\frac{d_{1}}{\lambda_{\infty}^{k} E(w, z)}+\cdots+\frac{d_{r}}{\lambda_{\infty}^{r k} E^{r}(w, z)}+\varepsilon_{r, k}(w, z)
$$

where $\left|\lambda_{\infty}^{(r+1) k} \varepsilon_{r, k}(w, z)\right| \leq \frac{P(|z|)}{|w|}$. For $r=1$, this has already been done above. For a given $r$, since by induction the result of Proposition 4.9 is true at step $l-1$, we may write

$$
\begin{aligned}
& \frac{1}{w(k)}-\frac{1}{\lambda_{\infty}^{k} E(w, z)}= \\
& \quad=\left(\frac{-a_{2}}{\lambda_{\infty}^{k} E(w, z)}-\cdots-\frac{a_{l-1}}{\lambda_{\infty}^{k(l-2)} E^{(l-2)}(w, z)}\right) \frac{1}{w(k)}+\frac{\delta_{k}(w, z)}{\lambda_{\infty}^{k} w(k) E(w, z)} .
\end{aligned}
$$

This last term goes into $\varepsilon_{r, k}(w, z)$ because $r \leq l-1$. We then use the inductive hypothesis to replace the term $\frac{1}{w(k)}$ which lies at the right handside of this inequality and we finish by collecting the terms in an appropriate way.

Lemma 4.11. Let $p \geq 1, q \geq 0$ and $j \geq 0$ with $p+q=j$. One has:

$$
\frac{z^{p}(k)}{w^{q}(k)}=\frac{d_{j}}{\lambda_{\infty}^{k j} E^{j}(w, z)}+\cdots+\frac{d_{l}}{\lambda_{\infty}^{l k} E^{l}(w, z)}+\delta_{p, q, k}(w, z)
$$

where $d_{i} \in \mathbb{C},\left|\lambda_{\infty}^{k \cdot \min (l+1, l+p+q-1)} \delta_{p, q, k}(w, z)\right| \lesssim_{M} \frac{P(|z|)}{|w|^{\min (1, q)}}$.

Proof. We write

$$
\begin{aligned}
& \frac{z^{p}(k)}{w^{q}(k)}=\frac{1}{w^{q}(k)}\left(z^{p}(k)-\left(\frac{b_{1}}{\lambda_{\infty}^{k} E(w, z)}+\cdots+\frac{b_{l-1}}{\lambda_{\infty}^{(l-1) k} E^{l-1}(w, z)}\right)^{p}\right)+ \\
& +\left(\frac{b_{1}}{\lambda_{\infty}^{k} E(w, z)}+\cdots+\frac{b_{l-1}}{\lambda_{\infty}^{(l-1) k} E^{l-1}(w, z)}\right)^{p}\left(\frac{1}{w^{q}(k)}-\frac{c_{q}}{\lambda_{\infty}^{k q} E^{q}(w, z)}\right. \\
& \left.\quad-\cdots-\frac{c_{l-1}}{\lambda_{\infty}^{(l-1) k} E^{l-1}(w, z)}\right) \\
& +\left(\frac{b_{1}}{\lambda_{\infty}^{k} E(w, z)}+\cdots+\frac{b_{l-1}}{\lambda_{\infty}^{(l-1) k} E^{l-1}(w, z)}\right)^{p}\left(\frac{c_{q}}{\lambda_{\infty}^{k q} E^{q}(w, z)}+\right. \\
& \left.\quad+\cdots+\frac{c_{l-1}}{\lambda_{\infty}^{(l-1) k} E^{l-1}(w, z)}\right) \\
& :=I+I I+I I I .
\end{aligned}
$$


The last term has exactly the desired form. The first one is estimated using the mean value theorem and inequality (4.8):

$$
|I| \lesssim_{M} \frac{1}{\lambda_{\infty}^{k q}|w|^{q}} \times \frac{P(|z|)}{\lambda_{\infty}^{l k}} \times \frac{Q(|z|)}{\lambda_{\infty}^{(p-1) k}}=\frac{R(|z|)}{|w|^{q} \lambda_{\infty}^{k(p+q+l-1)}}
$$

For the second term, one has:

$$
|I I| \leq \frac{1}{\lambda_{\infty}^{k p}|w|^{p}} \times \frac{P(|z|)}{\lambda_{\infty}^{k l}|w|} \leq \frac{P(|z|)}{\lambda_{\infty}^{k(l+1)}|w|}
$$

Let us now come back to step $l$ of Proposition 4.9. We write $w(n)$ as in (4.5). Fix $j \leq l-1$ and $p, q$ with $p+q=j$. Using the result of Lemma 4.10 if $p=0$ and that of Lemma 4.11 otherwise, one gets

$$
\begin{aligned}
& \lambda_{\infty}^{n} \sum_{k=n}^{+\infty} \frac{a_{p, q}}{\lambda_{\infty}^{k+1}} \frac{z^{p}(k)}{w^{q}(k)}= \\
& \quad=\lambda_{\infty}^{n} \sum_{k=n}^{+\infty} \frac{1}{\lambda_{\infty}^{k+1}}\left(\frac{d_{j}}{\lambda_{\infty}^{k j} E^{j}(w, z)}+\cdots+\frac{d_{l-1}}{\lambda_{\infty}^{(l-1) k} E^{l-1}(w, z)}+\delta_{p, q, k}(w, z)\right)
\end{aligned}
$$

where $\left|\delta_{p, q, k}(w, z)\right| \leq \frac{P(|z|)}{\lambda_{\infty}^{k l}}$. Now on the one hand one has for $r \in[1, l-1]$ :

$$
\lambda_{\infty}^{n-1} \sum_{k=n}^{+\infty} \frac{1}{\lambda_{\infty}^{k(r+1)} E^{r}(w, z)}=\frac{c}{\lambda_{\infty}^{r n} E^{r}(w, z)} .
$$

On the other hand we obtain

$$
\lambda_{\infty}^{n-1} \sum_{k=n}^{+\infty} \frac{\left|\delta_{p, q, k}(w, z)\right|}{\lambda_{\infty}^{k}} \leq \lambda_{\infty}^{n-1} \sum_{k=n}^{+\infty} \frac{P(|z|)}{\lambda_{\infty}^{k(l+1)}|w|} \leq \frac{P_{1}(|z|)}{\lambda_{\infty}^{n l}}
$$

The same type of inequality holds true for the remaining terms. For instance, suppose that $p+q=l$. Lemma 4.10 and 4.11 ensure that

$$
\left|\frac{z^{p}(k)}{w^{q}(k)}\right| \lesssim_{M} \frac{P_{1}(|z|)}{\lambda_{\infty}^{k l}|w|^{\min (1, q)}}
$$

whereas we have $\left|E_{p, q}(w(k), z(k))\right| \lesssim_{M} P_{2}(|z(k)|) \lesssim_{M} P_{3}(|z|)$. We thus obtain

$$
\begin{aligned}
\lambda_{\infty}^{n} \sum_{k=n}^{+\infty} \frac{\left|E_{p, q}(w(k), z(k))\right|}{\lambda_{\infty}^{k+1}} \frac{\left|z^{p}(k)\right|}{\left|w^{q}(k)\right|} & \lesssim_{M} \lambda_{\infty}^{n} P_{1}(|z|) P_{3}(|z|) \sum_{k=n}^{+\infty} \frac{1}{\lambda_{\infty}^{k(l+1)}} \\
& \leq \frac{Q(|z|)}{\lambda_{\infty}^{n l}}
\end{aligned}
$$


In the same vein, we have

$$
\begin{aligned}
\lambda_{\infty}^{n} \sum_{k=n}^{+\infty} \frac{\left|\varepsilon_{1}(w(k), z(k))\right|}{\lambda_{\infty}^{k+1}} & \lesssim_{M} \quad \lambda_{\infty}^{n} \sum_{k=n}^{+\infty} \frac{1}{\lambda_{\infty}^{k} \lambda_{\infty}^{k(m / 2-2)}|w|} \\
& \lesssim_{M} \quad \lambda_{\infty}^{n} \sum_{k=n}^{+\infty} \frac{1}{\lambda_{\infty}^{k l}} \leq \frac{P(|z|)}{\lambda_{\infty}^{n(l-1)}}
\end{aligned}
$$

Let us now consider $z(n)$ given by (4.6). Since we have a further term in the expansion of $w(n)$ we get immediately a further term in the expansion of $1 / w^{q}(n)$ given by Lemma 4.10 . On the other hand for a given $(u, v)$ with $v \neq 0, u \neq 0$ one has $u+v-1 \geq 1$ meaning that the estimation on the rest of Lemma 4.11 becomes

$$
\left|\lambda_{\infty}^{k(l+1)} \delta_{u, v, k}(w, z)\right| \leq \frac{P(|z|)}{|w|} .
$$

Therefore, for $u+v \leq l, v>0$ one has

$$
\begin{aligned}
& \mu_{\infty}^{n-1} \sum_{k=0}^{n} \frac{b_{u, v}}{\mu_{\infty}^{k}} \frac{z^{u}(k)}{w^{v}(k)}= \\
& \quad=\mu_{\infty}^{n-1} \sum_{k=0}^{n} \frac{1}{\mu_{\infty}^{k}}\left(\frac{d_{1}}{\lambda_{\infty}^{k} E(w, z)}+\cdots+\frac{d_{l}}{\lambda_{\infty}^{k l} E^{l}(w, z)}+\eta_{u, v, k}(w, z)\right)
\end{aligned}
$$

where $\left|\lambda_{\infty}^{k(l+1)} \eta_{u, v, k}(w, z)\right| \leq \frac{P(|z|)}{|w|}$. These terms have the desired form, since

$$
\left|\mu_{\infty}\right|^{n} \sum_{k=0}^{n} \frac{1}{\left|\mu_{\infty}\right|^{k+1}}\left|\eta_{u, v, k}(w, z)\right| \lesssim_{M} \max \left(\left|\mu_{\infty}\right|^{n}, 1 / \lambda_{\infty}^{k(l+1)}\right) \frac{P(|z|)}{|w|}
$$

For the other ones, we proceed exactly like for $w(n)$. Indeed, when $u+v=$ $l+1, v>0$, one has:

$$
\left|\mu_{\infty}\right|^{n} \sum_{k=0}^{n} \frac{\left|F_{u, v}(w(k), z(k))\right|}{\left|\mu_{\infty}\right|^{k+1}}\left|\frac{z^{u}(k)}{w^{v}(k)}\right| \lesssim_{M} \sum_{k=0}^{n} \frac{P(|z|)}{\left|\mu_{\infty}\right|^{k}|w| \lambda_{\infty}^{(l+1) n}}
$$

whereas the rest is estimated by:

$$
\begin{aligned}
\left|\mu_{\infty}\right|^{n} \sum_{k=0}^{n} \frac{1}{\left|\mu_{\infty}\right|^{k+1}}\left|\varepsilon_{2}(w(k), z(k))\right| & \lesssim_{M}\left|\mu_{\infty}\right|^{n} \sum_{k=0}^{n} \frac{1}{\left(\left|\mu_{\infty}\right| \lambda_{\infty}^{m / 2-1}\right)^{k}|w|} \\
& \lesssim_{M} \frac{\left|\mu_{\infty}\right|^{n}}{|w|}
\end{aligned}
$$

because of the choice of $m$. 
We are ready to finish the proof of Theorem 4.1. In fact, setting $l=$ $[m / 2]-1$, we have $\lambda_{\infty}^{l+1}\left|\mu_{\infty}\right|>1$. Looking carefully at the last step of Proposition 4.9 , we observe that, when we divide them by $1 / \mu_{\infty}^{n}$, the series appearing on the left handside of (4.11), (4.12) and (4.13) converge uniformly on $L_{M}$. This implies that $\frac{1}{\mu_{\infty}^{n}}\left(z(n)-\frac{b_{1}}{\lambda_{\infty}^{n} E(w, z)}-\cdots-\frac{b_{l}}{\lambda_{\infty}^{n l}} E^{l}(w, z)\right)$ converges uniformly on $L_{M}$ to a function $D$ which satisfies

$$
|D(w, z)-z| \leq \frac{P(|z|)}{|w|}
$$

In particular, $D$ is not a constant function. Moreover, if we compose by $\phi$, then we obtain

$$
\begin{aligned}
D \circ \phi(w, z) & =\lim _{n \rightarrow+\infty} \frac{1}{\mu_{\infty}^{n}}\left(z(n+1)-\frac{b_{1}}{\lambda_{\infty}^{n+1} E(w, z)}-\cdots-\frac{b_{l}}{\lambda_{\infty}^{(n+1) l} E^{l}(w, z)}\right) \\
& =\mu_{\infty} D(w, z) .
\end{aligned}
$$

We can observe that (4.14) shows that $D(w(n), z(n))=O(z(n))$ in this case.

\subsection{Linearization of the second coordinate, case of resonances}

To finish the study of the hyperbolic maps we have to examine the existence of "resonances" namely when there exists an integer $l$ such that $\lambda_{\infty}^{l} \mu_{\infty}=1$. In the proof of Proposition 4.9 this affects only two steps. For step $l-1$, a change appears only in the estimation (4.13). We now have

$$
\left|\mu_{\infty}\right|^{n} \sum_{k=0}^{n} \frac{1}{\left|\mu_{\infty}\right|^{k+1}}\left|\delta_{u, v, k}(w, z)\right| \lesssim\left|\mu_{\infty}\right|^{n} n \frac{P(|z|)}{|w|} .
$$

This implies that the majoration of $\eta_{n}(w, z)$ is slightly worse,

$$
\left|\eta_{n}(w, z)\right| \leq\left|\mu_{\infty}\right|^{n} n \frac{Q(|z|)}{|w|}
$$

In the last step, we have to take care of this in the evaluation of $z^{p}(k) / w^{q}(k)$. With the notations of Lemma 4.11, I becomes

$$
|I| \leq \frac{1}{\lambda_{\infty}^{k q}|w|^{q}} \times \frac{k P(|z|)}{\lambda_{\infty}^{l k}} \times \frac{Q(|z|)}{\lambda_{\infty}^{(p-1) k}}=\frac{k R(|z|)}{|w|^{q} \lambda_{\infty}^{k(p+q+l-1)}} .
$$

This means that we have to replace the condition on $\delta_{p, q, k}(w, z)$ by

$$
\left|\lambda_{\infty}^{k \cdot \min (l+1, l+p+q-1)} \delta_{p, q, k}(w, z)\right| \leq \frac{k P(|z|)}{|w|^{\min (1, q)}}
$$


When we report this on $w(n)$ this just multiplies the estimations obtained in (4.9) and in (4.10) by a factor $n$. This in turn gives $\left|\lambda_{\infty}^{(l-1) n} \delta_{n}(w, z)\right| \leq$ $n P(|z|)$. The most important change happens during the last step of the expansion of $z(n)$. Indeed one has now

$$
\mu_{\infty}^{n-1} \sum_{k=0}^{n-1} \frac{1}{\left(\mu_{\infty} \lambda_{\infty}^{l}\right)^{k} E^{l}(w, z)}=\mu_{\infty}^{n-1} \frac{n}{E^{l}(w, z)} .
$$

The rest does not change and we find that

$$
\frac{1}{\mu_{\infty}^{n}}\left(z(n)-\frac{b_{1}}{\lambda_{\infty}^{n} E(w, z)}-\cdots-\frac{b_{l} n}{\lambda_{\infty}^{n l} E^{l}(w, z)}\right)
$$

converges uniformly on $L_{M}$ to a function $D$ satisfying

$$
|D(w, z)-z| \lesssim_{M} \frac{P(|z|)}{|w|}
$$

$D$ is not constant and one has

$$
\begin{aligned}
D \circ \phi(w, z) & =\lim _{n \rightarrow+\infty} \frac{1}{\mu_{\infty}^{n}}\left(z(n+1)-\frac{b_{1}}{\lambda_{\infty}^{n+1} E(w, z)}-\cdots-\frac{b_{l} n}{\lambda_{\infty}^{(n+1) l} E^{l}(w, z)}\right) \\
& =\mu_{\infty} D(w, z)+\lim _{n \rightarrow+\infty} \frac{1}{\mu_{\infty}^{n}} \frac{b_{l}}{\lambda_{\infty}^{(n+1) l} E^{l}(w, z)} \\
& =\mu_{\infty} D(w, z)+\frac{b_{l}}{\lambda_{\infty}^{l} E^{l}(w, z)} .
\end{aligned}
$$

Thus we have obtained the following

Theorem 4.12. Let $\varphi \in \mathcal{D}^{2}\left(e_{1}\right)$ with $\lambda_{\infty}>1,0<\left|\mu_{\infty}\right|<1$ and $\alpha_{\infty} \neq 0$. Let $m$ be the least integer such that $\left|\mu_{\infty}\right| \lambda_{\infty}^{m / 2-1}>1$. We assume that $\varphi \in$ $\mathcal{D}^{m}\left(e_{1}\right)$ and we suppose also that there exists $l \in \mathbb{N}$ with $\lambda_{\infty}^{l} \mu_{\infty}=1$. Then there exist a holomorphic map $\sigma=(E, D): \mathbb{H}_{2} \rightarrow \mathbb{C}^{2}$ and a complex number $b$ such that

$$
\sigma \circ \phi(w, z)=\left(\lambda_{\infty} E(w, z), \mu_{\infty} D(w, z)+\frac{b}{E^{l}(w, z)}\right) .
$$

Moreover, there is a domain $\Omega$ such that, for any $(w, z) \in \mathbb{H}_{2}, \phi^{[n]}(w, z) \in \Omega$ for $n$ large enough and such that

$$
\begin{gathered}
E(w, z)-w=o(|w|) \text { when }(w, z) \rightarrow \infty \text { along } \Omega, \\
|D(w, z)-z| \leq \frac{P(|z|)}{|w|} \text { for }(w, z) \in \Omega
\end{gathered}
$$

where $P$ is a polynomial. Moreover, $E$ is the unique map with the previous properties and it exists under the weaker assumption $\varphi \in \mathcal{D}^{3}\left(e_{1}\right)$. 
Here, the model map is $(w, z) \mapsto\left(\lambda_{\infty} w, \mu_{\infty} z+\frac{b}{w^{l}}\right)$ which is not a linear fractional map. We can ask whether we can find a map $D$ such that $D \circ \phi=\mu_{\infty} D$. This is obviously the case: it suffices to take $D(w, z)=\frac{1}{E^{l}(w, z)}$. However, this does not seem to be an useful model. We would like to find a map $D$ which depends in a major way of $z$, namely a map $D$ satisfying something like $|D(w, z)-z| \leq \frac{|P(z)|}{|w|}$ on a domain $\Omega$ like above or $D(w(n), z(n))=O(z(n))$. This is generally impossible.

Example 4.13. Let $\phi(w, z)=(2 w+2, z / 2+1 /(w+1))$ which is a holomorphic self-map of $\mathbb{H}_{2}$. It is easy to prove that for a given $(w, z) \in \mathbb{H}_{2}$, one has

$$
\begin{aligned}
& w(n) \sim 2^{n}(w+2) \\
& z(n) \sim \frac{n}{2^{n-1}(w+2)} .
\end{aligned}
$$

If $D \circ \phi=\frac{1}{2} D$, then $D(w(n), z(n))=\frac{1}{2^{n}} D(w, z)$ whereas

$$
z(n) \sim \frac{n}{2^{n-1}(w+2)} \quad \text { and } \quad \frac{P(|z(n)|)}{|w(n)|} \lesssim \frac{1}{2^{n}|w+2|} .
$$

The two conditions $|D(w, z)-z| \leq \frac{P(|z|)}{|w|}$ or $D(w(n), z(n))=O(z(n))$ cannot be satisfied!

The fact that $\mu_{\infty} \lambda_{\infty}^{k}=1$ for $k \geq 1$ means that $\mu_{1}$ is resonant in the sense of Poincaré-Dulac (see [2]). Our work shows that all non-resonant terms can be annihilated, a situation similar to the classical Poincaré-Dulac theorem on differential equations.

\section{The parabolic diagonalizable case}

\subsection{The case $\left|\mu_{\infty}\right|<1-$ First coordinate}

The parabolic diagonalizable case seems to be the most difficult one. It can be divided into two subcases, provided $\left|\mu_{\infty}\right|<1$ (which is in some sense a mixing between parabolic and hyperbolic cases) or not. We first discuss the case $\left|\mu_{\infty}\right|<1$ and obtain a linearization under mild assumptions on $\varphi$. So, let $\varphi \in \mathcal{D}^{2}\left(e_{1}\right)$ with $\lambda_{1}=1,\left|\mu_{1}\right|<1$ and $\alpha_{1}=0$ (these assumptions on $\varphi$ will be kept throughout this subsection). As the proof of the hyperbolic case shows, the study of the quotients $\frac{z^{2}(n)}{w(n)+1}$ is crucial for our work. However, it turns out that it is difficult to obtain an analogue of Lemma 4.2 in the parabolic diagonalizable case. Indeed, since $\lambda_{\infty}=1$, there is nothing between 1 and $\lambda_{\infty}$ ! This explains in part why we are reduced to give supplementary conditions on $\phi$. 
We know by Wolff's lemma that for any $(w, z) \in \mathbb{H}_{2}$,

$$
\Re\left(\phi_{1}(w, z)\right)-\left|\phi_{2}(w, z)\right|^{2} \geq \Re(w)-|z|^{2} .
$$

We will assume that we have a stronger form of this inequality, namely that there exists $\delta>0$ such that, for any $(w, z) \in \mathbb{H}_{2}$,

$$
\Re\left(\phi_{1}(w, z)\right)-\left|\phi_{2}(w, z)\right|^{2} \geq \Re(w)-|z|^{2}+\delta .
$$

This assumption will play the same role than that made by Bourdon and Shapiro, when they require that a parabolic self-map $\psi$ of $\mathbb{C}_{+}$extends continuously to $\overline{\mathbb{C}_{+}}$and maps $\overline{\mathbb{C}_{+}}$into $\mathbb{C}_{+}$. Here, (5.1) ensures that $\Re(w(n)) \gtrsim n$. Our second condition is that the inequality in (2.3) is strict, namely

$$
\left|\mu_{\infty}\right|^{2}+\left|\gamma_{\infty}\right|<1
$$

As a matter of notation, we will say that $\phi$ satisfies the strong Wolff condition provided (5.1) and (5.2) hold true. Our result reads:

Theorem 5.1. Let $\varphi \in \mathcal{D}^{6+\varepsilon}\left(e_{1}\right)$ with $\lambda_{1}=1$ and $\alpha_{1}=0$. Suppose moreover that $\varphi$ satisfies the strong Wolff condition. There exist $a \in \mathbb{C}$ and $a$ holomorphic function $E: \mathbb{H}_{2} \rightarrow \mathbb{C}$ such that

$$
E \circ \phi=E+a \text {. }
$$

Moreover, for any $(w, z) \in \mathbb{H}_{2}$, one has $E(w(n), z(n))-w(n)=o(w(n))$.

The first step toward the proof of Theorem 5.1 is the analogue of Lemma 4.2.

Lemma 5.2. For any $(w, z) \in \mathbb{H}_{2}$, one has

$$
\frac{|z(n)|^{2}}{|w(n)+1|} \lesssim \frac{1}{n^{1 / 2}}
$$

Proof. As in Lemma 4.4, we write

$$
\frac{z(n+1)^{2}}{w(n+1)+1}=\frac{\mu_{\infty}^{2}}{1-\gamma_{\infty} \frac{z^{2}(n)}{w(n)+1}} \frac{z^{2}(n)}{w(n)+1}+O\left(|w(n)+1|^{-1 / 2}\right) .
$$

We set $\kappa=\frac{\left|\mu_{\infty}\right|^{2}}{1-\left|\gamma_{\infty}\right|}<1$. Since $\Re(w(n)) \gtrsim n$, there exists $A>0$ such that, for any $(w, z) \in \mathbb{H}_{2}$, one has

$$
\left|\frac{z^{2}(n+1)}{w(n+1)+1}\right| \leq \kappa\left|\frac{z^{2}(n)}{w(n)+1}\right|+\frac{A}{n^{1 / 2}} .
$$

Lemma 5.2 follows now from an easy induction.

This lemma allows us to control the behavior of $z(n)$ :

Lemma 5.3. There exists $0<\kappa<1$ such that for any $(w, z) \in \mathbb{H}_{2}$, one has $|z(n)| \lesssim \kappa^{n}|z|+\frac{1}{n^{1 / 2}}$. 
Proof. We proceed as in Section 4.4, writing

$$
\begin{aligned}
z(n)=\mu_{\infty}^{n} & \left(\prod_{j=0}^{n-1} \frac{1}{1-\gamma_{\infty} \frac{z^{2}(j)}{w(j)+1}} z+\sum_{j=0}^{n-1}\left(\prod_{k=j+1}^{n-1} \frac{1}{1-\gamma_{\infty} \frac{z^{2}(k)}{w(k)+1}}\right) \frac{1}{\mu_{\infty}^{j+1}} \times\right. \\
& \left.\left(\frac{z^{2}(j)}{w(j)+1} A(w(j), z(j))+\frac{z(j)}{w(j)+1} B(w(j), z(j))+\varepsilon(w(j), z(j))\right)\right)
\end{aligned}
$$

where $A, B$ are bounded on $\mathbb{H}_{2}$ and $|\varepsilon(w, z)| \lesssim \frac{1}{|w+1|}$. By Lemma 5.2, we may estimate the products appearing above. For $a, b, c$ three well-chosen positive numbers, one has

$$
\begin{aligned}
\prod_{k=j+1}^{n-1}\left|\frac{1}{1-\gamma_{\infty} \frac{z^{2}(k)}{w(k)+1}}\right| & \leq \prod_{k=j+1}^{n-1}\left(1+a\left|\frac{z^{2}(k)}{w(k)+1}\right|\right) \leq \prod_{k=j+1}^{n-1}\left(1+\frac{b}{k^{1 / 2}}\right) \\
& \leq \exp \left(\sum_{k=j+1}^{n-1} \frac{b}{k^{1 / 2}}\right) \lesssim \frac{\exp \left(c n^{1 / 2}\right)}{\exp \left(c(j+1)^{1 / 2}\right)}
\end{aligned}
$$

Now, for any $x>1$ and any $c>0$, there exists $C(x)$ such that

$$
\sum_{j=1}^{n} \frac{x^{j}}{\exp \left(c j^{1 / 2}\right) j^{1 / 2}} \leq C(x) \frac{x^{n}}{\exp \left(c n^{1 / 2}\right) n^{1 / 2}} .
$$

Indeed, we write

$$
\begin{aligned}
& \sum_{j=1}^{n} \frac{x^{j}}{\exp \left(c j^{1 / 2}\right) j^{1 / 2}}=\sum_{j=0}^{n-1} \frac{x^{n-j}}{\exp \left(c(n-j)^{1 / 2}\right)(n-j)^{1 / 2}} \\
& =\frac{x^{n}}{\exp \left(c n^{1 / 2}\right) n^{1 / 2}} \sum_{j=0}^{n-1} \frac{1}{x^{j}}\left(\frac{n}{n-j}\right)^{1 / 2} \exp \left(c n^{1 / 2}-c(n-j)^{1 / 2}\right) \\
& \leq \frac{x^{n}}{\exp \left(c n^{1 / 2}\right) n^{1 / 2}} \sum_{j=0}^{n-1} \frac{1}{x^{j}}(1+j) \exp \left(c j^{1 / 2}\right)
\end{aligned}
$$

and the last series is convergent. We come back to $z(n)$ and use the previous lemma to finish the estimation.

$$
\begin{aligned}
|z(n)| & \lesssim\left|\mu_{\infty}\right|^{n} \exp \left(c n^{1 / 2}\right)|z|+\left|\mu_{\infty}\right|^{n} \sum_{j=0}^{n} \frac{1}{\left|\mu_{\infty}\right|^{j+1}} \frac{\exp \left(c n^{1 / 2}\right)}{\exp \left(c(j+1)^{1 / 2}\right)} \frac{1}{(j+1)^{1 / 2}} \\
& \lesssim \kappa^{n}|z|+\frac{1}{n^{1 / 2}}
\end{aligned}
$$

where $\kappa$ is any positive number with $\kappa>\left|\mu_{\infty}\right|$. 
Lemma 5.3 gives immediately an improvement of the statement of Lemma 5.2, which becomes

$$
\frac{|z(n)|^{2}}{|w(n)+1|} \lesssim \frac{\kappa^{2 n}|z|^{2}+1 / n}{n+\Re(w)+1} \leq \kappa^{2 n}+\frac{1}{n^{2}}
$$

In turn, this estimation allows a dramatic enhancement of Lemma 5.2, since it shows that the infinite product

$$
\prod_{k=0}^{+\infty} \frac{1}{1-\gamma_{\infty} \frac{z^{2}(k)}{w(k)+1}}
$$

converges uniformly on the compact subsets of $\mathbb{H}_{2}$, with the estimation

$$
\prod_{k=j+1}^{n-1}\left|\frac{1}{1-\gamma_{\infty} \frac{z^{2}(k)}{w(k)+1}}\right| \lesssim 1
$$

Using the same induction formula for $z(n)$ and noting that

$$
\left|\frac{z^{2}(j)}{w(j)+1}\right||A(w(j), z(j))| \lesssim \frac{1}{j^{2}} \text { and }\left|\frac{z(j)}{w(j)+1}\right||B(w(j), z(j))| \lesssim \frac{1}{j^{3 / 2}},
$$

we finally find

$$
|z(n)| \lesssim\left|\mu_{\infty}\right|^{n}|z|+\frac{1}{n}
$$

Thus $|z(n)|$ behaves (at most) like $1 / n$ whereas $|w(n)|$ behaves (at least) like $n$. This invite us to proceed as in Section 4.6, namely to order the terms $\frac{z^{p}}{w^{q}}$ in the expansion of $\phi_{1}$ and $\phi_{2}$ near infinity with respect to the value of $p+q$. Hence, keeping in mind that

$$
d \varphi_{e_{1}}=\left(\begin{array}{cc}
1 & 0 \\
0 & \mu_{1}
\end{array}\right)
$$

if we suppose moreover that $\varphi \in \mathcal{D}^{6+\varepsilon}\left(e_{1}\right)$, we write for $|w|$ large enough

$$
\phi_{1}(w, z)=w+a+\sum_{p+q=1} a_{p, q} \frac{z^{p}}{(w+1)^{q}}+\sum_{p+q=2} \frac{z^{p}}{(w+1)^{q}} E_{p, q}(w, z)+\varepsilon_{1}(w, z)
$$

with $a, a_{p, q} \in \mathbb{C},\left|E_{p, q}(w, z)\right| \leq P(|z|), \operatorname{deg}(P) \leq 2,\left|\varepsilon_{1}(w, z)\right| \lesssim \frac{1}{|w|^{1+\varepsilon}}$ and

$$
\phi_{2}(w, z)=\mu_{\infty} z+\frac{b}{w+1}+\sum_{\substack{u+v=2 \\ v>0}} \frac{z^{u}}{(w+1)^{v}} F_{u, v}(w, z)+\varepsilon_{2}(w, z)
$$

with $b, b_{u, v} \in \mathbb{C},\left|F_{u, v}(w, z)\right| \lesssim 1,\left|\varepsilon_{2}(w, z)\right| \lesssim \frac{1}{|w|^{2+\varepsilon}}$. 
Lemma 5.4. There exists $0<\kappa<1$ such that, for any $(w, z) \in \mathbb{H}_{2}$, one has

$$
|w(n)-n a| \lesssim \kappa^{n}|z|^{4}+\log (n+1)+|w|
$$

Proof. We have

$$
\begin{aligned}
w(n)= & w+n a+\sum_{j=0}^{n-1} \sum_{p+q=1} a_{p, q} \frac{z^{p}(j)}{(w(j)+1)^{q}}+\sum_{p+q=2} E_{p, q}(w(j), z(j)) \frac{z^{p}(j)}{(w(j)+1)^{q}} \\
& +\varepsilon_{1}(w(j), z(j)) .
\end{aligned}
$$

The lemma follows now immediately from

$$
\left|\frac{z^{p}(j)}{w^{q}(j)}\right| \lesssim \frac{\left|\mu_{\infty}\right|^{p n}|z|^{p}+1 / n^{p}}{n^{q}} \text { and }\left|E_{p, q}(w(j), z(j))\right| \lesssim\left|\mu_{\infty}\right|^{2 n}|z|^{2}+\frac{1}{n^{2}}+1 .
$$

Remark 5.5. Lemma 5.4 implies $a \neq 0$ and even $\Re(a)>0$ since $\Re(w(n)) \gtrsim n$.

Lemma 5.6. For any $(w, z) \in \mathbb{H}_{2}$, for any $\delta>0$, one has

$$
\left|\frac{1}{w(n)+1}-\frac{1}{n a+1}\right| \lesssim \delta \frac{|z|^{4}+1+|w|}{\Re(w)^{1-\delta} n^{1+\delta / 2}} .
$$

Proof. This follows easily from Lemma 5.4 and the inequality $|w(n)| \geq$ $\Re(w(n)) \gtrsim \Re(w)+n$.

Lemma 5.7. For any $(w, z) \in \mathbb{H}_{2}$, one has

$$
\left|z(n)-b \mu_{\infty}^{n} \sum_{k=0}^{n-1} \frac{1}{\mu_{\infty}^{k+1}(k a+1)}\right| \lesssim \delta \frac{|z|^{4}+1+|w|}{\Re(w)^{1-\delta} n^{1+\delta / 2}} .
$$

Proof. We write

$$
\begin{aligned}
z(n)= & \mu_{\infty}^{n} z+\mu_{\infty}^{n}\left(\sum _ { k = 0 } ^ { n - 1 } \frac { 1 } { \mu _ { \infty } ^ { k + 1 } } \left(\frac{b}{w(k)+1}+\sum_{\substack{u+v=2 \\
v>0}} \frac{z^{u}(k)}{\left(w^{v}+1\right)(k)} F_{u, v}(w(k), z(k))\right.\right. \\
& \left.\left.+\varepsilon_{2}(w(k), z(k))\right)\right) .
\end{aligned}
$$

In view of Lemma 5.6, we replace $\frac{1}{w(k)+1}$ by $\frac{1}{k a+1}$. We estimate the difference by using

$$
\sum_{k=0}^{n-1} \frac{1}{\left|\mu_{\infty}\right|^{k+1}(k+1)^{1+\delta / 2}} \lesssim \frac{1}{\left|\mu_{\infty}\right|^{n} n^{1+\delta / 2}} .
$$

The remaining terms are handled as before. 
Proof of Theorem 5.1. We have

$$
\begin{aligned}
w(n)= & w+n a+\sum_{j=0}^{n-1} a_{1,0} z(j)+\sum_{j=0}^{n-1} \frac{a_{0,1}}{w(j)+1}+ \\
& +\sum_{j=0}^{n-1}\left(\sum_{p+q=2} E_{p, q}(w(j), z(j)) \frac{z^{p}(j)}{(w(j)+1)^{q}}+\varepsilon_{1}(w(j), z(j))\right) .
\end{aligned}
$$

Let

$$
Z(j)=b \mu_{\infty}^{j} \sum_{k=0}^{j-1} \frac{1}{\mu_{\infty}^{k+1}(k+1)} .
$$

Lemma 5.7 shows that the series $\sum_{j=0}^{+\infty} z(j)-Z(j)$ converges uniformly on the compact subsets of $\mathbb{H}_{2}$ with

$$
\left|\sum_{j=0}^{+\infty} z(j)-Z(j)\right| \lesssim \delta \frac{|z|^{4}+1+|w|}{\Re(w)^{1-\delta}} .
$$

Lemma 5.6 shows the same statement for $\sum_{j=0}^{n-1} \frac{1}{w(j)+1}-\frac{1}{j a+1}$ whereas the convergence and the estimations for the last terms have already been done in Lemma 5.4. We deduce that

$$
w(n)-n a-a_{1,0} \sum_{j=0}^{n-1} Z(j)-\frac{a_{0,1}}{a} \log (n a+1)
$$

converges uniformly on the compact subsets of $\mathbb{H}_{2}$ to a holomorphic function $E$ satisfying

$$
|E(w, z)-w| \lesssim \delta|z|^{4}+1+\frac{|w|}{\Re(w)^{1-\delta}} .
$$

Moreover, $E$ satisfies the functional equation $E \circ \phi=E+a$, since

$$
\begin{aligned}
E \circ \phi(w, z)= & \lim _{n \rightarrow+\infty}\left(w(n+1)-n a-\sum_{j=0}^{n-1} a_{1,0} Z(j)-\frac{a_{0,1}}{a} \log (n+1)\right) \\
= & \lim _{n \rightarrow+\infty}\left(w(n+1)-(n+1) a-\sum_{j=0}^{n} a_{1,0} Z(j)-\frac{a_{0,1}}{a} \log (n+2)\right) \\
& \quad+a+\lim _{n \rightarrow+\infty}\left(a_{1,0} Z(n)+\frac{\log (n+2)-\log (n+1)}{a} a_{0,1}\right) \\
= & E(w, z)+a .
\end{aligned}
$$

The last assertion concerning the behavior of $E$ follows easily from (5.3) and from the previous estimations on the behavior of $z(n)$ and $w(n)$. 


\subsection{The case $\left|\mu_{\infty}\right|<1$-Second coordinate}

As the formula for $\phi_{2}$ shows, we have here an induction formula which is similar to that of a hyperbolic map. However, there is a big difference: since $\lambda_{\infty}=1$, there does not exist an integer $k$ such that $\left|\mu_{\infty}\right| \lambda_{\infty}^{k} \geq 1$. In particular, if we consider the expansion of $z(n)$ the term $\mu_{\infty}^{n} z$ will always be smaller than $\frac{1}{(w(n)+1)^{p}}$ for any $p$. In particular, the regularity condition $\varphi \in \mathcal{D}^{m}\left(e_{1}\right)$ is not sufficient to understand completely the behavior of $z(n)$, even if $n$ is very large. Instead of it, we could expect to obtain a linear fractional model provided $\varphi$ is analytic at $e_{1}$. We mention here the work of T. Ueda [22] where the linearization of a holomorphic self-map of $\mathbb{C}^{2}$ has been studied at a fixed point where the differential has exactly the form studied here.

Unfortunately, even under the condition of analyticity, we have not been able to provide such a model. In particular, we do not succeed to manage at the same time the terms $\frac{1}{(w+1)^{q}}$ and the terms $\frac{z^{p}}{(w+1)^{q}}$ with $p \geq 1$. We give a partial answer with the linearization of three kinds of maps.

Example 5.8. Let $\varphi \in \mathcal{D}^{6}\left(e_{1}\right)$ with $\lambda_{1}=1, \alpha_{1}=0$ and suppose that $\phi$ satisfies the strong Wolff condition. Suppose moreover that $\phi_{2}$ may be written

$$
\phi_{2}(w, z)=\mu_{\infty} z+\sum_{j=1}^{d} \frac{a_{j}}{(w+1)^{j}} .
$$

Provided $\mu_{\infty}$ is not real, there exists $a \in \mathbb{C}, \Re(a)>0$ and two non-constant holomorphic functions $D, E: \mathbb{H}_{2} \rightarrow \mathbb{C}$ such that $(E, D) \circ \phi=\left(E+a, \mu_{\infty} D\right)$.

The existence of $E$ and $a$ is already given by Theorem 5.1. We set $\psi(w, z)=\left(w+a, \phi_{2}(w, z)\right)$. We have to be careful because we do not know whether $\mathbb{H}_{2}$ is preserved by $\psi$ or not. However, there exists a large enough $M$ such that the domain $E_{M}=\left\{(w, z) \in \mathbb{H}_{2}: \Re(w)>M\right.$ and $|z|^{2} \leq$ $\Re(w) / 2\}$ is mapped into itself by $\psi$. Let $(w, z)$ be in such a $U_{M}$ and set $(w(n), z(n))=\psi^{[n]}(w, z)$. We have

$$
z(n)=\mu_{\infty}^{n} z+\mu_{\infty}^{n-1} \sum_{k=0}^{n-1} \sum_{j=1}^{d} \frac{a_{j}}{(w+k a+1)^{j} \mu_{\infty}^{k}} .
$$

Let us introduce the Lerch transcendent function

$$
L(z, s, v)=\sum_{n=0}^{+\infty}(v+n)^{-s} z^{n}
$$

defined for $|z|<1$ and $v=0,1,2, \ldots$ (our reference for this subject is [15]). 
This function satisfies the functional equational, for any $m \geq 1$,

$$
L(z, s, v)=z^{m} L(z, s, m+v)+\sum_{n=0}^{m-1}(\nu+n)^{-s} z^{n} .
$$

An integral formula shows that for a fixed couple $(s, v)$ with $\Re(s)>0$ and $\Re(v)>0, L$ can be extended analytically in the first variable to $\mathbb{C} \backslash[1,+\infty[$. Of course, relation (5.4) remains true. Thus, provided $\mu_{\infty}$ is not real,

$$
\begin{aligned}
& \sum_{k=0}^{n-1} \frac{a_{j}}{(w+k a+1)^{j} \mu_{\infty}^{k}}=\sum_{k=0}^{n-1} \frac{a_{j}}{a^{j}\left(k+\frac{w+1}{a}\right)^{j} \mu_{\infty}^{k}} \\
& \quad=\frac{a_{j}}{a^{j}} L\left(1 / \mu_{\infty}, j,(w+1) / a\right)-\frac{a_{j}}{a^{j} \mu_{\infty}^{n}} L\left(1 / \mu_{\infty}, j,(w+1) / a+n\right) .
\end{aligned}
$$

In particular, the sequence

$$
\frac{z(n)+\sum_{j=1}^{+\infty} \frac{a_{j}}{a^{j} \mu_{\infty}} L\left(1 / \mu_{\infty}, j,(w+1) / a+n\right)}{\mu_{\infty}^{n}}
$$

converges uniformly on the compact subsets of $U_{M}$ to a holomorphic function $D_{1}$ satisfying $D_{1} \circ \psi=\mu_{\infty} D_{1}$. We transfer this property on $\phi$ in the following way. Let $(w, z) \in \mathbb{H}_{2}$. Using the asymptotic expansion of $E$, there exists an integer $p$ such that $\left(E\left(\phi^{[p]}(w, z)\right), \phi_{2}^{[p]}(w, z)\right) \in U_{M}$. We set

$$
D(w, z)=\frac{1}{\mu_{\infty}^{p}} D_{1}\left(E\left(\phi^{[p]}(w, z), \phi_{2}^{[p]}(w, z)\right)\right) .
$$

This does not depend on $p$ since

$$
D_{1}\left(E\left(\phi^{[p+1]}\right), \phi_{2}^{[p+1]}\right)=D_{1}\left(\psi\left(E\left(\phi^{[p]}\right), \phi_{2}^{[p]}\right)\right)=\mu_{\infty} D_{1}\left(E\left(\phi^{[p]}, \phi_{2}^{[p]}\right)\right) .
$$

The same argument shows that $D \circ \phi=\mu_{\infty} D$. The condition $\mu_{\infty}$ is not real is rather intriguing and irritating. It appears here just to extend the Lerch transcendent function.

Example 5.9. Let $\varphi \in \mathcal{D}^{6}\left(e_{1}\right)$ with $\lambda_{1}=1, \alpha_{1}=0$ and suppose that $\phi$ satisfies the strong Wolff condition. Suppose moreover that $\phi_{2}$ may be written

$$
\phi_{2}(w, z)=\mu_{\infty} z+\sum_{p=1}^{d} \sum_{q=1}^{d^{\prime}} \frac{a_{p, q^{2}} z^{p}}{(w+1)^{q}} .
$$

There exists $a \in \mathbb{C}, \Re(a)>0$ and two non-constant holomorphic functions $D, E: \mathbb{H}_{2} \rightarrow \mathbb{C}$ such that $(E, D) \circ \phi=\left(E+a, \mu_{\infty} D\right)$. 
As above, we may and shall assume that $\phi_{1}(w, z)=w+a$. We have

$$
z(n)=\mu_{\infty}^{n} \prod_{k=0}^{n-1}\left(1+\sum_{p=1}^{d} \sum_{q=1}^{d^{\prime}} \frac{a_{p, q}}{\mu_{\infty}^{k}} \frac{z^{p-1}(k)}{(w+k a+1)^{q}}\right) z .
$$

Using the results which appear during the proof of Theorem 5.1, we write

$$
1+\sum_{p=1}^{d} \sum_{q=1}^{d^{\prime}} \frac{a_{p, q}}{\mu_{\infty}^{k}} \frac{z^{p-1}(k)}{(w+k a+1)^{q}}=1+\frac{b}{w+k a+1}+R_{k}(w, z),
$$

where $\left|R_{k}(w, z)\right| \leq \frac{C(w, z)}{k^{2}}$. It is then easy to check that

$$
\prod_{k=0}^{n-1}\left(1+\sum_{p=1}^{d} \sum_{q=1}^{d^{\prime}} \frac{a_{p, q}}{\mu_{\infty}^{k}} \frac{z^{p-1}(k)}{(w+k a+1)^{q}}\right)=(w+n a+1)^{b} C_{n}(w, z)
$$

where $C_{n}(w, z)$ converges to a non-zero holomorphic function $C(w, z)$. It suffices now to set

$$
D(w, z)=\lim _{n \rightarrow+\infty} \frac{z(n)}{\mu_{\infty}^{n}(w+n a+1)^{b}} .
$$

Example 5.10. Suppose that $\phi(w, z)=\left(w+1, \mu_{\infty} z+\mu_{\infty} \frac{z}{w+1}+\frac{b}{w+1}\right)$. Then there exists a non-constant holomorphic function $D: \mathbb{H}_{2} \rightarrow \mathbb{C}$ such that $D \circ \phi=\mu_{\infty} D$.

By induction, one has

$$
\begin{aligned}
z(n) & =\mu_{\infty}^{n} \prod_{k=1}^{n}\left(1+\frac{1}{k+w+1}\right) z+b \sum_{j=0}^{n-1} \mu_{\infty}^{n-1-j} \prod_{k=j+1}^{n-1}\left(1+\frac{1}{k+w+1}\right) \frac{1}{w+j+1} \\
= & \mu_{\infty}^{n}(n+w+2) \frac{z}{w+2}+b \mu_{\infty}^{n-1}(n+w+1) \sum_{j=0}^{n-1} \frac{1}{\mu_{\infty}^{j}(w+j+1)(w+j+2)} \\
= & \mu_{\infty}^{n}(n+w+2) \frac{z}{w+2}+b \mu_{\infty}^{n-1}(n+w+1)\left(L\left(\frac{1}{\mu_{\infty}}, 1, w+1\right)\right. \\
& \left.-\frac{1}{\mu_{\infty}^{n}} L\left(\frac{1}{\mu_{\infty}}, 1, w+n+1\right)-L\left(\frac{1}{\mu_{\infty}}, 1, w+1\right)+\frac{1}{\mu_{\infty}^{n}} L\left(\frac{1}{\mu_{\infty}}, 1, w+n+1\right)\right) .
\end{aligned}
$$

$D$ is now defined by

$$
D(w, z)=\lim _{n \rightarrow+\infty} \frac{z(n)+b(n+w+1)\left(L\left(\frac{1}{\mu_{\infty}}, 1, w+n+1\right)-L\left(\frac{1}{\mu_{\infty}}, 1, w+n+2\right)\right)}{\mu_{\infty}^{n}(n+w+2)} .
$$

Of course, a miracle happens in this example: there is a simple expression for the product $\prod_{k=j-1}^{n}\left(1+\frac{1}{k+w+j}\right)$. If the coefficient before $\frac{z}{w+1}$ was not 
$\mu_{\infty}$, a major step toward the linearization of the second coordinate would be to have an analytic expansion of the function

$$
z \mapsto \sum_{k} \frac{\Gamma(w+k+u)}{\Gamma(w+k)} \frac{z^{k}}{k+w+1}
$$

(exactly like for the Lerch transcendent function). However, this would not solve the problem if there existed other terms $\frac{z^{p}}{(w+1)^{q}}$ with $p \geq 1$.

Question 5.11. Under the conditions of Theorem 5.1 and $\varphi$ analytic at $e_{1}$, does there exist a holomorphic and non-constant function $D: \mathbb{H}_{2} \rightarrow \mathbb{C}$ such that $D \circ \phi=\mu_{\infty} D$ ?

\subsection{The case $\left|\mu_{\infty}\right|=1$}

The second subcase occurs when $\left|\mu_{\infty}\right|=1$. The situation here seems quite difficult. Indeed the behavior of $z(n)$ and of $z^{2}(n) / w(n)$ can be very different, and of course very different from the model map.

Example 5.12. Let $\phi(w, z)=\left(w+1+\frac{2 z^{2}}{5 w}, z+\frac{3 z}{5 w}\right)$ which is a self-map of $\mathbb{H}_{2}$. Let $(w, z) \in \mathbb{H}_{2}$ with $w>0, z>0$ so that $w(n), z(n)>0$ for any $n$. It can be easily shown that $\frac{z^{2}(n+1)}{w(n+1)}>\frac{z^{2}(n)}{w(n)}$ for $n$ large enough. In particular, $\frac{z^{2}(n)}{w(n)}$ converges to $l \in(0,1]$. We then obtain that $w(n) \sim\left(1+\frac{2 l}{5}\right) n$. Now, one has

$$
\begin{aligned}
\frac{z^{2}(n+1)}{w(n+1)} & =\frac{z^{2}(n)}{w(n)}\left(1+\left(\frac{1}{5}-\frac{2 z^{2}(n)}{5 w(n)}\right) \times \frac{1}{w(n)}+O\left(\frac{1}{n^{2}}\right)\right) \\
& \sim \frac{z^{2}(n)}{w(n)}\left(1+\frac{1-2 l}{5} \times \frac{1}{(1+2 l / 5) n}+O\left(\frac{1}{n^{2}}\right)\right) .
\end{aligned}
$$

Since $z^{2}(n) / w(n)$ converges to a non-zero limit, one has necessarily $l=1 / 2$. This in turn implies that $w(n) \sim \frac{6}{5 n}$ and $z(n) \sim \sqrt{\frac{6}{10}} n^{1 / 2}$. The linearization for the second coordinate will be obtained by taking $\lim _{n} z(n)-\sqrt{\frac{6}{10}} n^{1 / 2}$.

Example 5.13. Let $\phi(w, z)=\left(w+1, z+\frac{1}{10(w+1)}\right)$ which is a self-map of $\mathbb{H}_{2}$. One has $w(n)=w+n$ and $z(n)=z+\sum_{k=1}^{n} \frac{1}{10(w+k)}$. If we suppose that $w>0$, one has $\sum_{k=1}^{n} \frac{1}{(w+k)}=\log (n+w)+G_{n}(w)$ where $G_{n}(w)$ converges when $n$ goes to infinity. In particular, one has $z(n) \simeq \frac{\log (n)}{10}$. The linearization for the second coordinate has to be obtained by considering $\lim _{n} z(n)-\frac{\log (n)}{10}$. 
Example 5.14. Let $\phi(w, z)=\left(w+1, z+\frac{\lambda z}{w}\right)$ which is a self-map of $\mathbb{H}_{2}$ for $\lambda \in[0,1 / 2)$. Let $(w, z) \in \mathbb{H}_{2}$ with $w>0, z>0$. We get $w(n)=w+n$ and

$$
z(n)=z \prod_{k=0}^{n-1}\left(1+\frac{\lambda}{w+k}\right)=z H_{n}(w)(n+w)^{\lambda}
$$

where $H_{n}(w)$ converges to $H(w)$. In particular, $z(n) \sim(n+w)^{\lambda} z H(w)$. The linearization for the second coordinate can be obtained by considering $\lim _{n} \frac{z(n)}{(n+w)^{\lambda}}$.

The three previous examples show the complexity of this subcase and the (at least) three different behaviors which lead to a different way for linearizing the second coordinate. Hence, we ask the following question:

Question 5.15. Does there exist a linear fractional model for the parabolic diagonalizable case with $\mu_{\infty}=1$ under weak assumptions on $\varphi$ ?

\section{Applications}

\subsection{Commuting self-maps of the ball}

In this section, we apply the theory developed above to the problem of commuting holomorphic mappings without fixed point in the ball. We show how the existence of a linear fractional model shed some light on this problem. It turns out that two maps which do not have the same model cannot commute.

Theorem 6.1. Let $\varphi, \psi$ be holomorphic self-maps of $\mathbb{B}_{2}$. Suppose that $\varphi$ and $\psi$ do not have any fixed point in $\mathbb{B}_{2}$ and let $P_{\varphi}, P_{\psi}$ their respective Denjoy-Wolff point. We assume that $\varphi$ and $\psi$ satisfy one of the following three conditions.

1. $\varphi$ is of parabolic non-diagonalizable type, $\varphi \in \mathcal{D}^{2}\left(P_{\varphi}\right), \psi$ is of parabolic non-diagonalizable type with $\mu_{1}^{\psi} \neq 1, \psi \in \mathcal{D}^{2}\left(P_{\psi}\right)$.

2. $\varphi$ is of parabolic non-diagonalizable type, $\varphi \in \mathcal{D}^{2}\left(P_{\varphi}\right), \psi$ is of hyperbolic type, $\psi \in \mathcal{D}^{2}\left(P_{\psi}\right)$.

3. $\varphi$ is of parabolic diagonalizable type with $\left|\mu_{1}^{\varphi}\right|<1$, $\varphi$ satisfies the strong Wolff condition and $\varphi \in \mathcal{D}^{6+\varepsilon}\left(P_{\varphi}\right), \psi$ is of hyperbolic type, $\psi \in \mathcal{D}^{2}\left(P_{\psi}\right)$.

Then $\varphi$ and $\psi$ do not commute. 
Proof. We argue by contraction and suppose that $\varphi \circ \psi=\psi \circ \varphi$. Suppose that $P_{\varphi} \neq P_{\psi}$ and, without loss of generality, that $P_{\varphi}=e_{1}$ and that $d \varphi_{e_{1}}$ has its reduced form. From a result of [7] we know that $\varphi$ and $\psi$ fix (as a set) the complex slice joining the two different Wolff points and that they are hyperbolic automorphisms of this slice. Let $v$ be the complex direction of this slice, $v \notin e_{1}^{\perp}=\mathbb{C} e_{2} . v$ is an eigenvector of $d \varphi_{e_{1}}$ corresponding to an eigenvalue of modulus smaller than 1. This cannot happen if $\varphi$ is of parabolic non-diagonalizable type, since the spectrum of $d \varphi_{e_{1}}$ is $\{1\}$. This does not hold also for the parabolic diagonalizable type since now the eigenspaces are $\mathbb{C} e_{1}$ corresponding to the eigenvalue 1 , and $\mathbb{C} e_{2}$. Hence, $P_{\varphi}=P_{\psi}=e_{1}$. We set $\phi=U \circ \varphi \circ U^{-1}$ and $\Psi=U \circ \psi \circ U^{-1}$. We distinguish the three cases:

1. One has $d \varphi_{e_{1}} d \psi_{e_{1}}=d \psi_{e_{1}} d \varphi_{e_{1}}$ which can only happen if $d \psi_{e_{1}}$ is a scalar multiple of the identity. This is not true.

2. The same argument shows that one has necessarily $\mu_{1}=\lambda_{1}$ which contradicts $\left|\mu_{1}\right|^{2} \leq \lambda_{1}$ and $\lambda_{1}>1$.

3. We know that there exists a map $E: \mathbb{H}_{2} \rightarrow \mathbb{C}$ and $a \in \mathbb{C}, a \neq 0$ so that $E \circ \phi=E+a$. Since $\phi$ and $\Psi$ commute, we get

$$
E \circ \Psi \circ \phi^{[n]}=E \circ \Psi+n a .
$$

In particular, one has $E \circ \Psi \circ \phi^{[n]}(1,0) \sim n a$. On the other hand, using the asymptotic expansion of $E$ given by (5.3) and the expansion of $\psi$, one has

$$
\begin{aligned}
E \circ \Psi \circ \phi^{[n]}(1,0) & \sim \Psi_{1} \circ \phi^{[n]}(1,0) \\
& \sim \lambda_{\infty}^{\Psi} n a .
\end{aligned}
$$

This is a contradiction since $\lambda_{\infty}^{\Psi} \neq 1$.

Remark 6.2. We can observe that above not only is the existence of a linear fractional model important, but also the behavior of the intertwining map $\sigma$ plays a crucial role. This illustrates the fact that the more information one has on $\sigma$, the more useful the model is.

\subsection{Supercyclic composition operators}

We end up this paper with an application of the linear fractional model for the unit disk to the dynamics of composition operators. Let us first recall several definitions from this very active field of operator theory.

Definition 6.3. An operator $T$ on a Banach space $X$ is said hypercyclic if there exists a vector $x \in X$ such that its orbit $\operatorname{Orb}(T, x)=\left\{T^{n} x ; n \geq 0\right\}$ is dense in $X$. 
Definition 6.4. An operator $T$ on a Banach space $X$ is said supercyclic if there exists a vector $x \in X$ such that its projective orbit $\operatorname{Orb}(T, x)=$ $\left\{c T^{n} x ; n \geq 0, c \in \mathbb{C}\right\}$ is dense in $X$.

If $\varphi$ is a self-map of the unit disk $\mathbb{D}$ without any fixed point in $\mathbb{D}$, the dynamics of the composition operator $C_{\varphi}$ defined by $C_{\varphi}(f)=f \circ \varphi$ has been intensively studied by Bourdon and Shapiro in [6] and by Gallardo and Montes in [16]. Their results on the Hardy space $H^{2}(\mathbb{D})$ are:

- If $\varphi$ is a hyperbolic linear fractional map of $\mathbb{D}$ or a parabolic automorphism, $C_{\varphi}$ is hypercyclic on $H^{2}(\mathbb{D})$ whereas it is not if $\varphi$ is a parabolic linear fractional map which is not an automorphism. Via the linear fractional model, these conclusions remain true if $\varphi$ is a self-map of parabolic or hyperbolic type which is sufficiently regular at the DenjoyWolff point (see [6]).

- If $\varphi$ is a linear fractional map of parabolic non-automorphism type, then $C_{\varphi}$ is not even supercyclic (see [16]).

We show in this section how the linear fractional model of Bourdon and Shapiro allows us to extend the result of Gallardo and Montes to a large class of self-maps. Suppose that $\varphi$ is a parabolic self-map of $\mathbb{D}$ whose DenjoyWolff point is 1 and has $C^{3+\varepsilon}$-smoothness at 1 (the relative definition is given in [6]). We know that $\Re\left(\varphi^{\prime \prime}(1)\right) \geq 0$. If we suppose that $\varphi^{\prime \prime}(1) \neq 0$, the condition $\Re\left(\varphi^{\prime \prime}(1)\right)>0$ is equivalent to the fact that $\varphi$ is of parabolic non-automorphism type. Such maps are best understood on the right halfplane $\mathbb{C}_{+}=\{w \in \mathbb{C}: \Re(w)>0\}$ via the Cayley map $\sigma(z)=\frac{z+1}{1-z}$. Setting $\phi=\sigma \circ \varphi \circ \sigma^{-1}$, one has

$$
\phi(w)=w+a+\frac{b}{w+1}+\Gamma(w+1)
$$

where $a=\varphi^{\prime \prime}(1), b=-\frac{2}{3}(S \varphi)(1)$ ( $S \varphi$ is the Schwarzian derivative of $\varphi$ ) and $|\Gamma(w+1)| \leq \frac{1}{|w+1|^{\varepsilon+1}}, \varepsilon>0$. We suppose moreover that $\phi$ extends continuously to $\overline{\mathbb{C}_{+}}$and that $\phi\left(\overline{\mathbb{C}_{+}}\right) \subset \mathbb{C}_{+}$. We may also assume that $\phi$ is univalent (otherwise $C_{\varphi}$ is not even cyclic). In this case, Theorem 4.12 of [6] gives the existence of a holomorphic map $\nu: \mathbb{C}_{+} \rightarrow \mathbb{C}$ satisfying

- $\nu \circ \phi=\nu+a$.

- $\nu$ is univalent.

- $\nu$ may be written $\nu(w)=w-\frac{b}{a} \log (1+w)+B(w)$ with $B$ a bounded function. 
We assume moreover that $\Re(b / a) \geq 0$ (or equivalently $\Re \overline{\varphi^{\prime \prime}(1)}(S \varphi)(1) \leq 0$ ). We are going to prove that $C_{\varphi}$ is not supercyclic on $H^{2}(\mathbb{D})$. On the halfplane, this means that $C_{\phi}$ is not supercyclic on $\mathcal{H}=\left\{f \circ \sigma^{-1}: f \in H^{2}(\mathbb{D})\right\}$ endowed with $\left\|f \circ \sigma^{-1}\right\|_{\mathcal{H}}=\|f\|_{H^{2}(\mathbb{D})}$. We need the following elementary lemma.

Lemma 6.5. There exists $\alpha \in \mathbb{C}$ such that $\nu\left(\mathbb{C}_{+}\right) \supset \mathbb{C}_{\alpha}=\{w \in \mathbb{C}: \Re(w)>\alpha\}$.

Proof. Fix $w \in \mathbb{C}_{+}$and $z \in \mathbb{C}$. The equation $\nu(w)=z$ may be rewritten

$$
w=z+\frac{b}{a} \log (1+w)+B(w):=z+f(w) .
$$

Now, it is easy to check that $\Re\left(\frac{b}{a} \log (1+w)\right)=\Re(b / a) \log |1+w|+C(w)$ where $C$ is a bounded function. In particular, our assumption $\Re(b / a) \geq 0$ ensures the existence of $\beta \in \mathbb{R}$ such that $\Re\left(\frac{b}{a} \log (1+w)+B(w)\right) \geq \beta$. Moreover, it is clear that $f^{\prime}(w)$ goes to 0 as $\Re(w)$ grows to $\infty$. Thus there exists $\gamma \in \mathbb{R}$ such that $\Re(w) \geq \gamma \Longrightarrow\left|f^{\prime}(w)\right| \leq \frac{1}{2}$. To conclude, it suffices to observe that if the real part of $z$ is large enough, then $w \mapsto z+f(w)$ maps $\mathbb{C}_{\gamma}$ into itself and to apply the fixed point theorem.

Of course, if $\nu$ is a solution to $\nu \circ \phi=\nu+a$, then $\nu-\alpha$ remains a solution. The previous lemma says that we may assume that $\overline{\mathbb{C}_{+}} \subset \nu\left(\mathbb{C}_{+}\right)$.

We are going to inverse the linear fractional model (this is the main novelty of this part of the proof). Denoting $\tau_{a}(w)=w+a$ and $\sigma=\nu_{\mid \mathbb{C}_{+}}^{-1}$, the linear fractional relation reads

$$
\nu \circ \phi=\tau_{a} \circ \nu .
$$

We compose by $\sigma$ at the right handside to get:

$$
\nu \circ \phi \circ \sigma=\tau_{a} .
$$

This implies in particular that for any $w \in \mathbb{C}_{+}, \nu \circ \phi \circ \sigma(w)$ belongs to $\mathbb{C}_{+}$. A composition by $\sigma$ at the left handside gives

$$
\phi \circ \sigma=\tau_{a} \circ \sigma .
$$

Moreover, $\sigma(i \mathbb{R})$ is a Jordan curve. A standard argument now ensures that the supercyclicity of $C_{\phi}$ would imply that of $C_{\tau_{a}}$, which is not supercyclic by the result of Gallardo and Montes. For the sake of completeness, we give the details. Suppose that $C_{\phi}$ is supercyclic on $\mathcal{H}$, and let $f$ be a supercyclic vector for $C_{\phi}$. Let $u$ be in $\mathcal{H}$, and $\varepsilon>0$. Since $\sigma(i \mathbb{R})$ is a Jordan curve, Walsh Theorem shows that $C_{\sigma}$ has dense range. So, let $v \in \mathcal{H}$ with $\|u-v \circ \sigma\|<\varepsilon$. There exists $n_{p} \in \mathbb{N}$ and $\lambda_{p} \in \mathbb{C}$ such that

$$
\left\|\lambda_{p} C_{\phi^{\left[n_{p}\right]}} f-v\right\|<\varepsilon .
$$


Applying $C_{\sigma}$ we get

$$
\left\|\lambda_{p} f \circ \phi^{\left[n_{p}\right]} \circ \sigma-v \circ \sigma\right\|<\varepsilon\left\|C_{\sigma}\right\| .
$$

The use of the inverse linear fractional relation gives

$$
\left\|\lambda_{p}(f \circ \sigma) \circ \tau_{n_{p} a}-u\right\|<\varepsilon+\varepsilon\left\|C_{\sigma}\right\| .
$$

This shows that $f \circ \sigma$ is a supercyclic vector for $C_{\tau_{a}}$, a contradiction. Summarizing the previous discussion, we have proven the following.

Theorem 6.6. Let $\varphi$ be a self-map of $\mathbb{D}$, whose Denjoy-Wolff point is 1 , satisfying $\varphi^{\prime}(1)=1, \Re\left(\varphi^{\prime \prime}(1)\right)>0$ and $\Re\left(\overline{\varphi^{\prime \prime}(1)}(S \varphi)(1) \leq 0\right.$. Suppose moreover that $\varphi$ extends continuously to $\overline{\mathbb{D}}$ with $\varphi(\mathbb{T} \backslash\{1\}) \subset \mathbb{D}$. Then $C_{\varphi}$ is not supercyclic on $H^{2}(\mathbb{D})$.

Remark 6.7. The condition $\Re\left(\overline{\varphi^{\prime \prime}(1)}(S \varphi)(1) \leq 0\right.$ already appears in [6]. It was the most difficult case to prove that the operator $C_{\varphi}$ is cyclic. It is not surprizing that it is the most easy case to prove that $C_{\varphi}$ is not supercyclic. Of course, we may ask whether Theorem 6.6 remains true without this condition.

On the ball, the situation concerning linear dynamics is not so clear even for composition operators with linear fractional symbols. There are (positive) results for the case of two boundary fixed points in [5] and the following negative result in [4]: if $\varphi$ is a generalized Heisenberg translation, not an automorphism, then $C_{\varphi}$ is not hypercyclic on $H^{2}\left(\mathbb{B}_{2}\right)$. Our work suggests the following natural question:

Question 6.8. Let $\varphi: \mathbb{B}_{2} \rightarrow \mathbb{B}_{2}$ be holomorphic, whose linear fractional model is a generalized Heisenberg translation, not an automorphism. Is it true that $C_{\varphi}$ is not hypercyclic on $H^{2}\left(\mathbb{B}_{2}\right)$ ?

\section{References}

[1] AbAte, M.: Iteration theory of holomorphic maps on taut manifolds. Research and Lecture Notes in Mathematics. Complex Analysis and Geometry. Mediterranean Press, Rende, 1989

[2] Arnold, V. I.: Geometrical methods in the theory of ordinary differential equations. Grundlehren der Mathematischen Wissenschaften 250. SpringerVerlag, New-York, 1983.

[3] Baker, I. N. And Pommerenke, Ch.: On the iteration of analytic functions in a half-plane II. J. London Math. Soc. (2) 20 (1979), 255-258. 
[4] Bayart, F.: A class of linear fractional maps of the ball and their composition operators. Adv. Math. 209 (2007), 649-665.

[5] Bisi, C. AND Bracci, F.: Linear fractional maps of the unit ball: a geometric study. Adv. in Math. 167 (2002), 265-287.

[6] Bourdon, P. And Shapiro, J. H.: Cyclic phenomena for composition operators. Mem. Amer. Math. Soc. 125 (1997), no. 596.

[7] Bracci, F.: Common fixed points of commuting holomorphic maps in the unit ball of $\mathbb{C}^{n}$. Proc. Amer. Math. Soc. 127 (1999), 1133-1141.

[8] Bracci, F., Contreras, M. and Díaz-Madrigal, S.: Classification of semigroups of linear fractional maps in the unit ball. Adv. Math. 208 (2007), no. 1, 318-350.

[9] Bracci, F. And Gentili, G.: Solving the Schröder equation at the boundary in several variables. Michigan Math. J. 53 (2005), 337-356.

[10] Bracci, F. and Poggi-Corradini, P.: On Valiron's theorem. In Future trends in geometric function theory, 39-55. Rep. Univ. Jyväskylä Dep. Math. Stat. 92, Univ. Jyväskylä, Jyväskylä, 2003.

[11] Contreras, M., Díaz-Madrigal, S. and Pommerenke, Ch.: Some remarks on the Abel equation in the unit disk. J. London Math. Soc. (2) 75 (2007), 623-634.

[12] Cowen, C.: Iteration and the solution of functional equations for functions analytic in the unit disc. Trans. Amer. Math. Soc. 265 (1981), 69-95.

[13] Cowen, C. And MacCluer, B.: Linear fractional maps of the ball and their composition operators. Acta. Sci. Math. (Szeged), 66 (2000), 351-376.

[14] Cowen, C. And MacCluer, B.: Schroeder's equation in several variables. Taiwanese J. Math. 7 (2003), no. 1, 129-154.

[15] Erdélyi, A., Magnus, W., Oberhettinger, F. and Tricomi, F.: Higher transcendental functions. Vol I. McGraw-Hill Book Company, Inc., New York-Toronto-London, 1953.

[16] Gallardo-Guttiérez, E. and Montes-Rodríguez, A.: The role of the spectrum in the cyclic behavior of composition operators. Mem. Amer. Math. Soc. 167 (2004), no. 791.

[17] Königs, G.: Recherches sur les intégrales de certaines équations fonctionnelles. Ann. Sci. École Norm. Sup. (3) 1 (1884), 3-41.

[18] MacCluer, B.: Iterates of holomorphic self-maps of the unit ball in $\mathbb{C}^{N}$. Michigan Math. J. 30 (1983), 97-106.

[19] Pommerenke, Ch.: On the iteration of analytic functions in a halfplane. J. London Math. Soc. (2) 19 (1979), 439-447.

[20] Rudin, W.: Function theory in the unit ball of $\mathbb{C}^{n}$. Fundamental Principles of Mathematical Science 241. Springer-Verlag, New York-Berlin, 1980.

[21] Sternberg, S.: Local contractions and a theorem of Poincaré. Amer. J. Math. 79 (1957), 809-824. 
[22] UEDA, T.: Local structure of analytic transformations of two complex variables. I. J. Math. Kyoto Univ. 26 (1986), 233-261.

[23] Valiron, G.: Sur l'itération des fonctions holomorphes dans un demi-plan. Bull. Sci. Math. (2) 55 (1931), 105-128.

Recibido: 8 de marzo de 2007

Revisado: 1 de octubre de 2007

Frédéric Bayart

Laboratoire de Mathématiques

Université Blaise Pascal

Campus des Cézeaux

F-63177 Aubiere Cedex France

Frederic.Bayart@math.univ-bpclermont.fr 